\title{
UNDERSTANDING THE RELATIONSHIP BETWEEN GLOBAL AND DIVERSITY LEARNING PRACTICE TYPES, CRITICAL THINKING AND AWARENESS OF SELF AND OTHERS IN COLLEGE STUDENTS
}

\author{
A Dissertation presented to \\ the Faculty of the Educational Leadership and Policy Analysis Department \\ at the University of Missouri-Columbia \\ In Partial Fulfillment \\ of the Requirements for the Degree \\ Doctor of Education \\ by \\ Jennifer L. Wiley \\ Dr. Ximena Uribe-Zarain, Dissertation Supervisor \\ July 2018
}


(C) Copyright by Jennifer L. Wiley 2018

All Rights Reserved 
The undersigned, appointed by the dean of the Graduate School, have examined the dissertation entitled

UNDERSTANDING THE RELATIONSHIP BETWEEN GLOBAL AND DIVERSITY LEARNING PRACTICE TYPES, CRITICAL THINKING AND AWARENESS OF SELF AND OTHERS IN COLLEGE STUDENTS

presented by Jennifer L. Wiley, a candidate for the degree of Doctor of Education, and hereby certify that, in their opinion, it is worthy of acceptance.

Professor Ximena Uribe-Zarain

Professor Leslie Anderson

Professor Cynthia MacGregor

Professor Denise Baumann 
For Kristofor Robin, my source of strength and endurance; for Ellie and Cora who are strong women, already; and for my father who believed I could. 


\section{ACKNOWLEDGEMENTS}

I would first like to thank my advisor, Dr. Ximena Uribe-Zarain. Her guidance and encouragement led me to a positive place for learning and endurance. I would also like to acknowledge the sweat equity and perseverance of my committee members: Dr. Cynthia MacGregor, Dr. Leslie Anderson, and Dr. Denise Baumann, for their hours of reading, patient advice, and endless support. For access to BEVI dataset and kind encouragement, I would like to thank Dr. Craig Shealy. For the hours of discussions, guidance and support on Transformative Learning Theory, I would like to offer my gratitude to Dr. John Dirkx and Dr. Kris Acheson-Clair. And to Dr. Kristofor Wiley my sincerest thanks for guidance and support through the magical land of statistical analysis. 


\section{TABLE OF CONTENTS}

ACKNOWLEDGEMENTS ............................................................................................ ii

LIST OF FIGURES .......................................................................................................................... vi

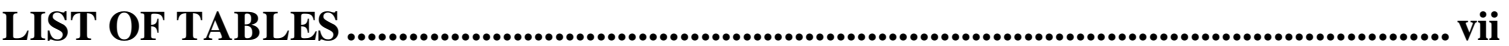

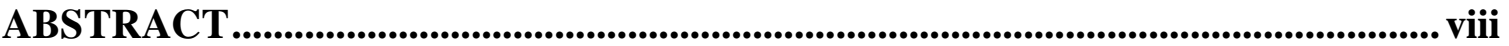

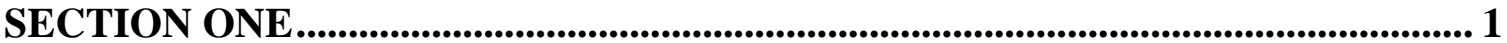

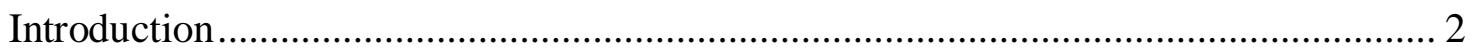

Background of the Study .......................................................................................... 3

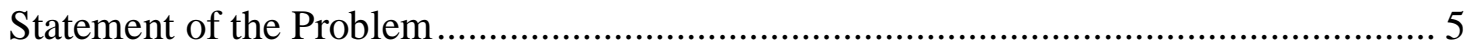

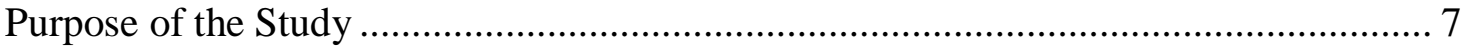

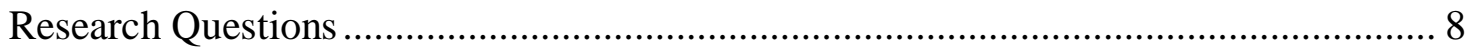

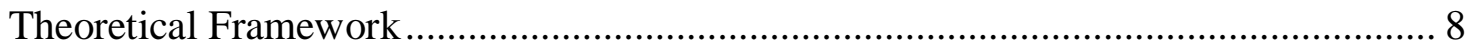

Definitions for Theoretical Framework ……………............................................... 10

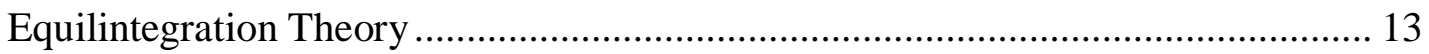

Transformative Learning Theory ……………………...................................... 17

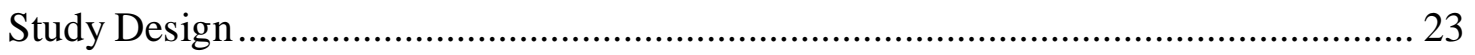

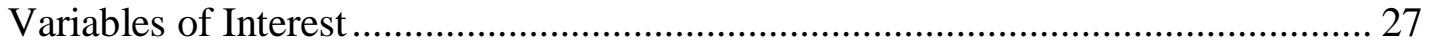

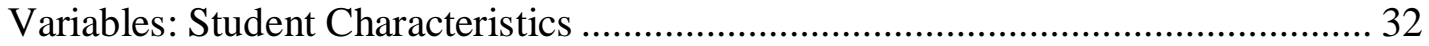

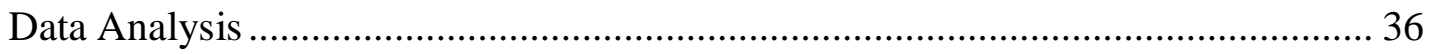

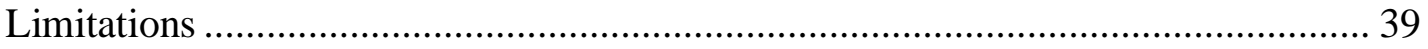

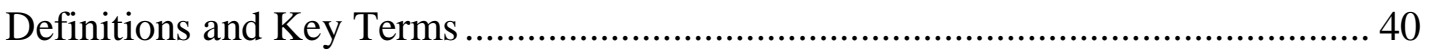

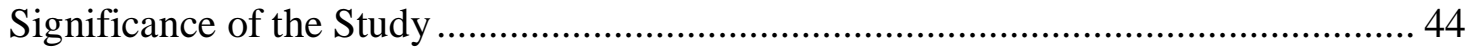

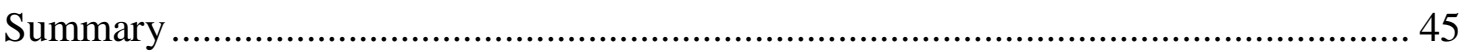




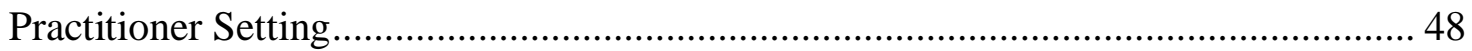

History of GDLs in US Higher Education ........................................................... 51

Organizational Analysis .................................................................................. 56

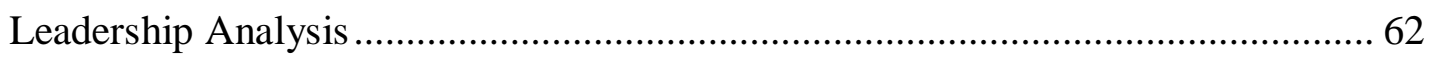

Implications for Research in the Practitioner Setting .............................................. 67

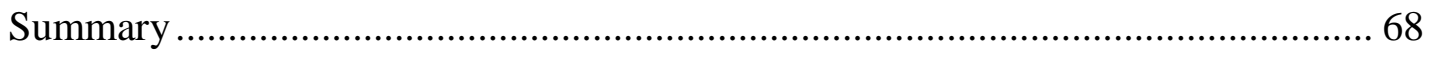

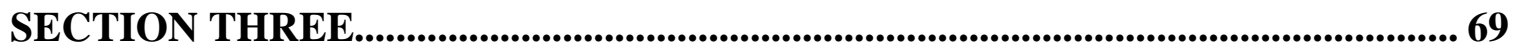

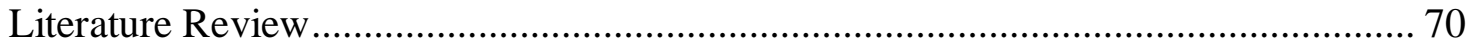

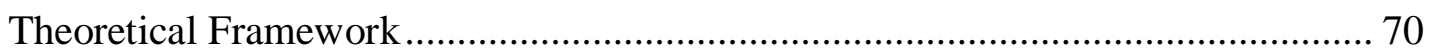

High Impact Practice: Rationale and Definitions .................................................... 74

Global and Diversity Learning Programs ............................................................... 76

Outcomes Related to GDL Practice Types ………………....................................... 80

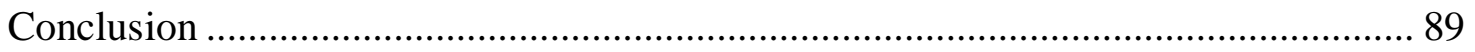

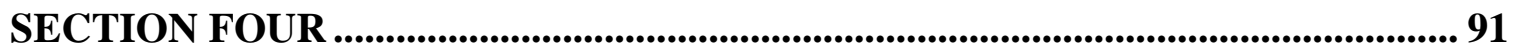

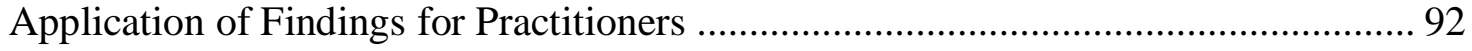

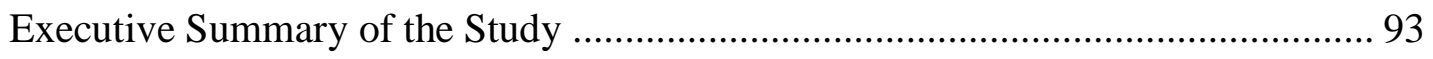

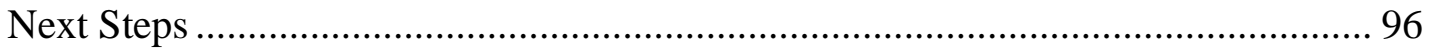

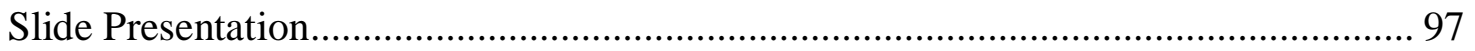

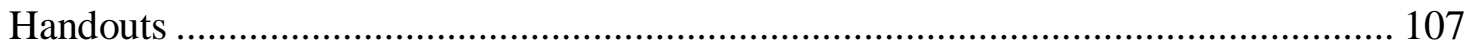

SECTION FIVE .............................................................................................................. 110

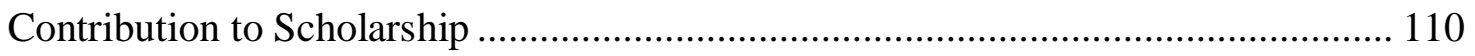




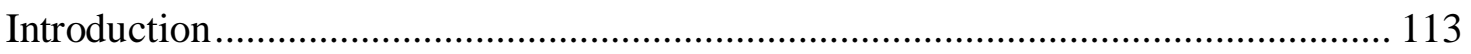

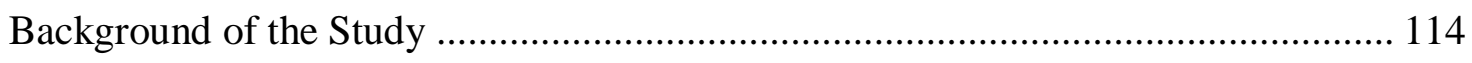

Theoretical Framework .................................................................................. 118

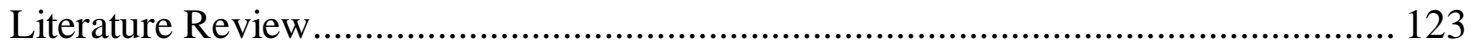

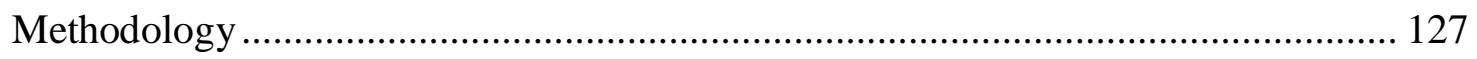

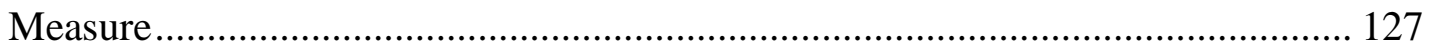

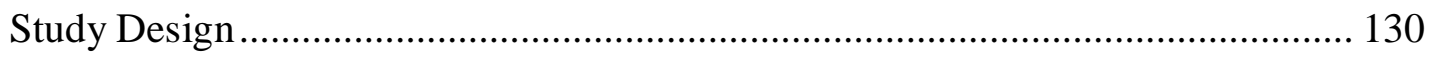

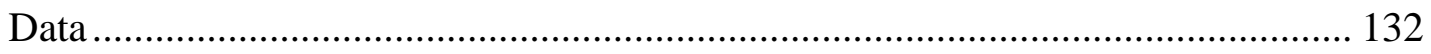

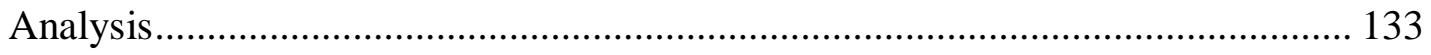

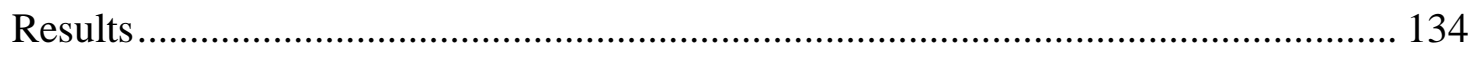

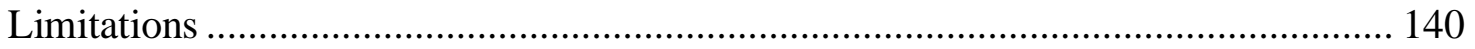

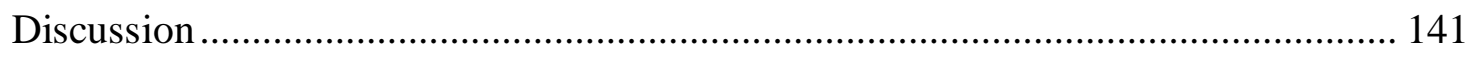

Mechanisms Underlying Gender Difference .................................................. 143

Implications for Program Implementation ............................................... 145

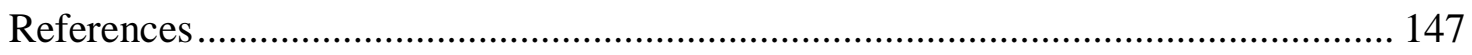

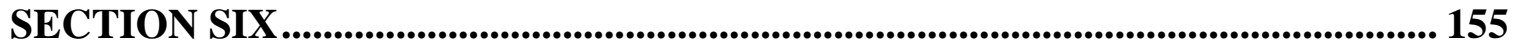

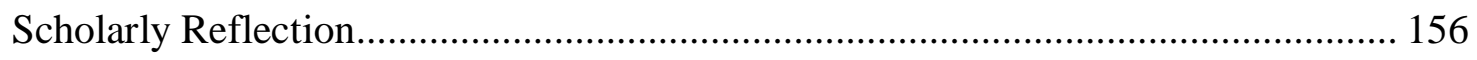

REFERENCES............................................................................................................... 163

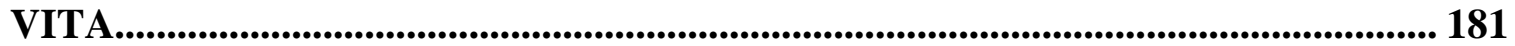




\section{LIST OF FIGURES}

Figure 1. A pictographic model of the EI Self (Shealy, 2015). ................................... 16

Figure 2. Self Access regression model example ................................................... 38

Figure 3. Mintzberg's Organizational Model in the Context of GDL Practices .............. 58

Figure 4. StrengthsFinder (Rath, 2007) top five traits pre-program and post-program. 157 


\section{LIST OF TABLES}

Table 1 Alignment of Theoretical Framework to Practice and Measures .................... 19

Table 2 Domains and Scales of the BEVI ............................................................ 26

Table 3 Descriptions of Global and Diversity Practice Types .................................... 28

Table 4 Descriptions of Key Variables of Interest................................................... 34

Table 5 Alignment of Theoretical Framework to Practice Measures ........................ 122

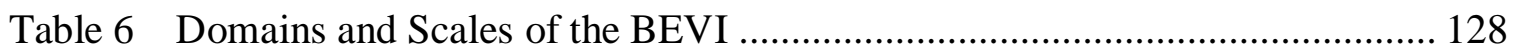

Table 7 Descriptions of Global and Diversity Program Types ................................ 131

Table 8 Sample Demographic Frequencies ........................................................... 133

Table 9 Mean Outcome Scores at Time 1 Administration by Practice Type ............. 135

Table 10 Summary of Block Regression Analysis for Variables Predicting Education

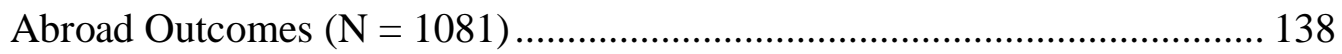

Table 11 Summary of Block Regression Analysis for Variables Predicting Global

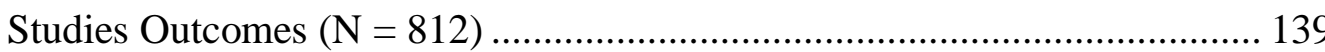




\begin{abstract}
The ability to think critically and complexly amid novel experiences that require self- and other-awareness is something that leaders in an array of social institutions seek to develop in their communities. Global and Diversity Learning (GDL) practices, one category of high impact practices (Kuh, 2008), aim to increase students' awareness of self and others and imbue critical thinking skills that will help students see how their own background and experiences interact with those of peers. This study aims to understand the relationship of four GDL practices (education abroad, multicultural programming, intercultural living-learning communities, and global studies coursework) to desired learning outcomes. Through completing ANCOVAs and multiple regression analyses on an existing dataset of GDL participants, this study demonstrates the influence of gender, socio-economic status, and citizenship within GDL practice types. The study findings also reveal significant differences between group members' other awareness and critical thinking capacity. Understanding how different GDL practice types affect capacity development will allow university leadership to direct collaboration between departments and align programming, to allocate resources more effectively, and communicate potential outcomes based on empirical data.
\end{abstract}


SECTION ONE

INTRODUCTION TO THE STUDY 


\section{Introduction}

"In terms of cultural and political matters, you are likely to be described as accepting, open, liberal, and progressive. Such ways of thinking and being can be wonderful, as you likely anticipate eagerly an encounter with ideas..." She quietly folded the paper, peered from the corner of her eye to see if anyone else was watching, pulled her arms across her body tightly, and slid down in her chair to wait for the debrief session to begin. It only took one word in her diagnostic report to create the same amount of internal conflict in Anna's mind as her combined experiences studying abroad that semester. At the beginning of the group debrief, she raised her hand, suggested that the validity of the instrument was akin to a Vanity Fair magazine quiz, and categorically rejected the idea that she was "liberal." Her group mates giggled warmly and confirmed that she was the lone "conservative," but she was unequivocally their conservative. The facilitator asked Anna and her group mates to talk about what it meant to be politically liberal or conservative in both their host community and a US context. A healthy discussion ensued, but Anna drifted into the background. Toward the end of the debrief session, the facilitator asked Anna if she felt comfortable sharing what she was thinking. Anna replied, “I do not want to go home, because I won’t fit in anymore. None of my friends or family will understand my experiences here." After studying abroad in Chile for a semester, Anna had been quietly reflecting on what it would mean if her way of seeing the world had changed. She was going through a process of cognitive disequilibrium. The process takes place when one's understanding of the way the world functions and new experiences or information are incongruous (Mezirow, 1997; Piaget, 1952; Shealy, 2015). For Anna, her understanding of identity and political affiliation met 
with new contrasting information she could not ignore. She felt a sense of belonging and shared some beliefs with a group of people, who prior to studying abroad, were merely "the liberal other." As she began to make meaning with this new information, she became somewhat distressed. What would happen if she had changed? How would her needs for affiliation and connection be met when she went home? Could the study abroad program have been designed to better prepare her for or support her in this internal conflict? What would have been the ideal outcome for Anna, the program organizers, or the university that promoted her study abroad experience? Is this the type of outcome university administrators and faculty members are after when they talk about high impact practices?

\section{Background of the Study}

The ability to think critically and with complexity in the face of novel or unexpected information, like Anna's experience, that require self and emotional awareness are outcome goals that military, corporate and civic leaders are seeking to develop in their respective populations (Abbe, Herman, \& Gulik, 2007; Gardner, Gross, \& Steglitz, 2008; Kuh, 2005). Response to demand for these skills began to grow in US higher education through initiatives like the American Association of Colleges and Universities' (AAC\&U) Liberal Education and America’s Promise (LEAP) campaign, launched in 2005. The campaign sought to develop 21 st century skills in university students - particularly those who have not traditionally had access to college (Brownell \& Swaner, 2010). LEAP was intended to help universities keep up with the pace of change in the workforce by organizing national discussions about the current needs of employers, establishing a research agenda to provide evidence of the outcomes associated 
with a liberal arts education, and setting up a public advocacy campaign to promote liberal education to business leaders and policy makers (Brownell \& Swaner, 2010).

Essential learning outcomes identified by LEAP were those which would begin early in the student's college experience then build and develop across their educational experience. Knowledge of human cultures and the natural world, intellectual and practical skills, ability to recognize responsibility for self and others, and ability to apply learning were the core categories of learning identified through the initiative (Maki \& Schneider, 2015). What universities needed was a system of experiences that targeted these specific outcomes because many of these skills and understandings are not targets of conventional content courses within programs of study.

To meet US university needs for programming that would facilitate development of these targeted 21st century skills, AAC\&U commissioned several literature reviews and studies to determine what works in higher education. Ten overarching categories of high impact practices (HIPs) emerged as the most effective mechanisms for developing 21st skills: (a) global and diversity learning, (b) first-year seminars, (c) common intellectual experiences, (d) learning communities, (e) writing-intensive courses, (f) undergraduate research, (g) collaborative projects, (h) service learning, (i) internships, and (j) capstone projects. These experiences had an added bonus of increasing student performance overall — the potential to close achievement gaps if participation by minority students could be increased (Brownell \& Swaner, 2010; Maki \& Schneider, 2015).

One category of HIPs, global and diversity learning practices (GDLs), specifically targets competencies that are needed to address achievement gaps. According to Sandeen (2012), GDLs should expand beyond the traditional international education focused 
boundary. She suggested that global and diversity learning should encourage students to think about social justice struggles in a more complex manner. Students should encounter differences that will lead them to discomfort and reflection on the origins of their worldview.

\section{Statement of the Problem}

In addition to being activities used by a select group of students, HIPs have faced more criticism because there has been little empirical evidence for achievement of learning outcomes (Brownell \& Swaner, 2010). Kuh et al. (2015) also cautioned that while there is some evidence for a small number of specific programs, administrators have been hasty to assign the label of high impact without enough empirical data to justify the ingredients on label.

Empirical data that does exist suggests that HIPs such as living-learning communities, education abroad, and undergraduate research programs can increase retention rates in undergraduate populations (Astin, 1993; Kuh, 2008; Provencher \& Kassel, 2017). Nevertheless, existing implicit value for HIPs like education abroad and the complexity of assessing learning in those experiences led universities to increase funding for certain types of GDL practices without the empirical evidence for the individual learning outcomes targeted through the LEAP initiative (Connor-Linton, Paige, \& Vande Berg, 2009). High impact learning initiatives like GDL practices are complex, multi-component experiences whose individual contribution to changes in student cognitive and emotional outcomes like self- and other-awareness are difficult to isolate. The internal outcomes for individual students, such as self-awareness, are less understood because the variables increase when one considers who a student is prior to 
the learning experience. Wandschneider and her colleagues (2015) noted that isolating the contribution of any practice toward significant change in a student's worldview is also particularly challenging because of its entanglement with the individual's internal variables like prior experiences and internal dialogues. In the current, polarizing climate in the US, with many campuses grappling with how to create cohesion amid rising tension over race and national identity, it is imperative that the academic community explore how these high impact initiatives change students' ways of perceiving themselves and interacting with others in the world.

With support for reflection and guidance to make meaning of new information, people can reorganize their perspectives to see the world more complexly and act more effectively to create sustainable internal systems (Shealy, 2015). Because globalization creates such opportunities and challenges for society, universities devote considerable resources to providing pathways for students to explore the world and the people in it. This research project is focused on one of the ten categories of HIPs that represent those resource expenditures, global and diversity learning practices

(GDLs). Administrators in US higher education need to understand which GDL practices most effectively facilitate desired 21st century learning outcomes in different students. The current body of knowledge on GDL practices, however, is inadequate for administrators to make decisions upon because most studies have focused on academic success outcomes, e.g., GPA or graduation rates (Kuh, 2008). 


\section{Purpose of the Study}

The purpose of this study was to understand which types of high impact GDL practices are most closely related to transformative learning outcomes that educational leaders desire: increased awareness of self and others as well as advanced critical and complex thinking. These three constructs were central to multiple reports listing $21 \mathrm{st}$ century skills established by industry and civic organizations (British Council, 2013; Connell, 2016; Hart Research Associates, 2008; Wilson-Mulnix, 2012). In 2011, US National Research Council (Pellegrino \& Hilton, 2012) reviewed literature and worked with a wide array of organizations to establish a shared definition of 21 st century skills. The Council's efforts established three overarching domains of competencies: (a) cognitive, (b) interpersonal, and (c) intrapersonal. While the outcomes of various types of GDL practices are represented in disparate scholarly literature, the interactions between student-characteristic variables and GDL practice types have not been thoroughly investigated (Wandschneider, Pysarchik, Sternberger, \& Ma, 2015). One reason for this gap in the literature is the lack of a method for operationalizing variables. Another reason for the gap is a lack of attention to understanding who students are before they participate in a given program preventing educators from understanding what sort of change may have occurred (Engle \& Engle, 2004; Wandschneider et al., 2015). This study operationalized four GDL practice variables and explored their relationship with the three intra- and interpersonal development outcomes established by the National Research Councils. 


\section{Research Questions}

This study addressed one gap in the literature by using a pre-existing dataset collected from a variety of GDL practices to answer the following research questions:

1) Are there differences between the types of GDL practices in terms of changes in college students' awareness of self and others and critical thinking (as measured by the Beliefs, Events and Values Inventory [BEVI])?

2) What are the relationships between college student characteristics, types of GDL practices and changes in awareness of self and other and critical thinking (as measured by the BEVI)?

\section{Theoretical Framework}

"When we started this program, I thought that people were born gay, but now I am not so sure. At home (in Central Asia), I am a minority, and I can't get a good job because of the way I was born. When I talk to other friends from near my home country in this program, they say that being gay is a choice that will ruin our family structures. What do you think?" This expression from Dalir, a graduate student, to his professor took place at the culmination of a two-week long intensive, multinational Model UN experience. The conversation provides a brief phenomenological example of how students enter GDL practices, and how their pre-existing worldviews and needs interact with their peers, as well as experiences and content that are prepared for them. The learning objectives for this program targeted content acquisition on the United Nation's efforts to protect human rights based upon sexual orientation and gender identity. How was Dalir's experience in the program interacting with his values and beliefs to facilitate or impeded learning? 
This snapshot from a sample GDL practice provides a context for the framework of this study. Why do students learn and change or resist learning and change? When students do learn, what exactly is it that changes? How could faculty and administrators who design GDL practice benefit from understanding who a student is prior to participation in a GDL practice? The following section will outline the theories that create the foundation for this study, keeping Dalir's experience present and a reminder of the applied context of learning.

Because of their many contexts and interdisciplinary nature, perhaps, high impact practices, like GDL practices, do not share a common theoretical framework. Scholars concerned with HIPs have applied a variety of theoretical frames from Critical Theory (Kilgo, 2016), to the Developmental Model of Intercultural Sensitivity (Connor-Linton et al., 2009; Engle \& Engle, 2004; Stebleton, Soria, \& Cherney, 2013) to Global Citizenship (Friedman, Haverkate, Oomen, Park, \& Sklad, 2015). Authors may bring their own disciplinary background and training to explain why they expect certain outcomes from these types of practices.

In the discussion which follows, two complementary theoretical frameworks are put to use to explain how GDL practices could facilitate change in students' selfawareness, other-awareness, and critical thinking. The first theoretical frame, Equilintegration (EI) Theory (Shealy, 2004, 2006, 2015) provides the study with a foundation for how people initially develop and maintain or change the what they believe about themselves and the world around them. The second theoretical frame, Transformative Learning Theory (Dirkx, 1998; Hoggan, 2016; Mezirow, 1997) is used to 
describe the layers within the individual that change which provide a bridge to pedagogy that can inform GDL practices.

\section{Definitions for Theoretical Framework}

To efficiently bridge these theories and avoid confusing jargon, definitions of frequently used constructs are provided. While these terms are understood in common vernacular, it is important to provide their contextual meaning here. A more extensive list of definitions relevant to the entire study follows later in this chapter.

Beliefs. There are many scholars who have extensively studied these constructs: Dabrowski (1964), Dirkx (2012), Feather (1992), Kahemann (2003), Rokeach (1973), Schwartz (1992), and Shealy (2015) are just a few, but their work is influential in framing this study. For the purpose of this discussion, an accessible definition is appropriate for creating a shared understanding of how beliefs and values connect to the constructs of self-awareness, other-awareness, and critical thinking. Broadly speaking, beliefs are an individual's ideas about what is true based on her or her prior and current interactions and inputs from the context in which he or she lives (Shealy, 2015). It is possible for people to share a belief but from a different perspective and to a different degree than others. Dalir, for example, entered his GDL experience believing that people should not be discriminated against for characteristics with which they are born, in part, because of his own experiences with discrimination based upon his ethnicity. Sam, an undergraduate student from the US from a majority culture profile, shared the same belief but more from a background of growing up in a context where equality and self-expression were valued by his parents. His experience with discrimination was abstract while Dalir's was concrete, changing the nature and perspective of how he might internalize the belief. 
Values. One may hold many beliefs, and those that are held and maintained over time solidify into values. Beliefs are the building blocks of values and are, thus, fewer in number than beliefs (Feather, 1992; Rokeach, 1973; Shealy, 2015). Feather (as cited in Shealy, 2015) states that,

Values can be conceived as abstract structures that involve the beliefs that people hold about desirable ways of behaving or about desirable end states. These beliefs transcend specific objects and situations, and they have a normative, or oughtness quality about them. (p. 45)

At a basic level, values connect and interact with one another as people interact with their environment. How they make sense of the input they receive becomes a system.

Worldview. The worldview represents the internal system for organizing, connecting, and making meaning of beliefs and values. Like beliefs and values, a person's way of making meaning of the inputs from his or her environment may not be apparent to him or her. There is an unconscious screening process that is constantly at work in people in order to keep them internally balanced, integrated between what they believe about the world and the inputs from the world (Shealy, 2015). It is through reflection and interacting with new information that a person can become aware of the filtering process, asking, "Why do I believe that?" This process is often uncomfortable and can lead to disequilibrium, simply stated, a sense that the world does not make sense in the same way it did. Therefore, worldview as a concept is important to the study because a worldview is shaped through an awareness of how one's self and relationships with others meet one's core needs. For the purpose of this study, a worldview is a system of beliefs and values. 
Need. Theorists and researchers involved in understanding beliefs and values often interact with the construct of need because of the foundational influence it has on the formation of beliefs and values. A need, at a basic level, is an interaction or input that a person suffers, physiologically or psychologically, without. When human needs go unmet long enough, it shapes the way an individual takes in and processes information about the environment, thus affecting belief formation (Shealy, 2015).

Self. The simplicity of the word appears disconnected from its definition. Shealy (2015) points to an irony that the construct of "self" is one of the most widely researched constructs in the field of psychology while sharing no commonly understood definition among scholars. A useful definition for the context of this study comes from Hungarian psychologist Mikayli Csikszentmihalyi (as cited in Shealy, 2015),

The self. . .contains everything else that has passed through consciousness: all the memories, actions, desires, pleasures, and pains are included in it. And more than anything else, the self represents the hierarchy of goals that we have built up, bit by bit, over the years. . .however much we are aware of it, the self is in many ways the most important element of consciousness, for it represents symbolically all of consciousness's other contents, as well as the pattern of their interrelations. (p. 34)

This definition points to the complexity of the composition of humans. It also envelops the entangled nature of a person's accumulated experiences, how and whether those experiences meet the person's needs, and, ultimately, how the person perceives his or her environment. 
Two theories are useful for examining the outcomes of GDL practices. The first, Equilintegration (EI) Theory, helps explain how students enter a GDL practice, from a psychological readiness perspective, and why they may or may not resist learning or change. EI Theory (Shealy, 2004, 2006, 2015) provides the framework for understanding how students develop the way they see themselves and the worldviews that they bring to interventions like GDL practices. EI theory draws connections between the many facets of who a person is, how needs are met, and how input from the external environment shapes their system of beliefs and values, or worldview. It provides a framework for understanding why interventions may have different outcomes for different students. The second, Transformative Learning Theory provides a bridge to the pedagogical design of GDL practices. Transformative Learning Theory describes what specific aspects of a student should change in order to claim an experience has sustained, high impact on student learning outcomes. The Transformative Learning framework established by Hoggan (2016) could allow educators to design GDL practices that would target change in those specific elements of who students are and how they see, or do not see, themselves and the world.

\section{Equilintegration Theory}

EI Theory seeks to explain "the processes by which beliefs, values, and worldviews are acquired and maintained, why their alteration is typically resisted, and how and under what circumstances their modification occurs" (Shealy, 2004, p. 1075). To begin that process of explanation, an understanding is warranted of who a person is and how they become who he or she is. An element of EI Theory is that while beliefs and values moderate behavior at individual and group levels, those beliefs and values 
may not be rational or known explicitly (Shealy, 2006). EI Theory outlines a developmental model that begins with infants acquiring an understanding of the world through the context that surrounds them (Shealy, 2004). Individuals develop layers of complexity as they begin to think of themselves as differentiated from caregivers and have internal dialogues, taking stimulus from the external environment, and making meaning from it. Shealy (2015) explained that ...belief and value content (i.e., the beliefs and values that a human being holds to be self-evident about self, others and the world at large) largely is a function of those beliefs and values that predominate in the primary cultures and context in which that human being develops and lives. (p. 96)

Through this framework, one begins to understand how students come to learning experiences with a particular worldview, or system of acquiring beliefs and values. Individuals are composed of beliefs and values that are available to them as they develop (Shealy, 2004; Vygotsky, 1997). Being unaware that they have a worldview, through lack of availability to contrasting views, may prevent students from being able to learn or change. Through novel experiences, that are not consonant with their prior experiences, individuals may gain awareness of their own beliefs and values and those of others. For true change in awareness or critical thinking to occur, some sort of intervention may be necessary to act as a catalyst (Dabrowski, 1964; Dirkx, 1998; Dweck, 2008; Mezirow, 1997). This can disturb a sense of balance the self has created regarding how the world makes sense (Shealy, 2015), therefore, GDL practices should be designed with support for reflection. 
A visual representation of EI Theory is provided in Figure 1 to help apply the EI theory to GDL practices. EI theory, in a simplistic description, maintains that as small infants (represented by the circles on the far left of the Figure 1), individuals develop worldviews gradually through iterative interactions with the outside world that seek to facilitate meeting core needs. Vygotsky (1997) notes how children pick up their caregivers' understandings, interpretations, and language for the inputs of their surroundings. As children gain experience and have their needs met, or not, through those interactions with the world around them, beliefs begin to form about who they are and how their world works. Over time, beliefs that are maintained, through consistently meeting a need or lack of new information, cluster with one another to form a value. Systems of connection and organization of beliefs are represented by clusters of boxes with darker boxes representing strongly held values in Figure 1. Lines from boxes represent the systems present for connecting beliefs to core needs, represented by the inner circle of each of the three models of self. When new information comes into conflict with existing values one line of the lines that connected a value to a core need disintegrates and a new connection is forged. Through processes of reflection and dialogue, students can reestablish the connections between a newly organized value, set of beliefs, and the core need. 


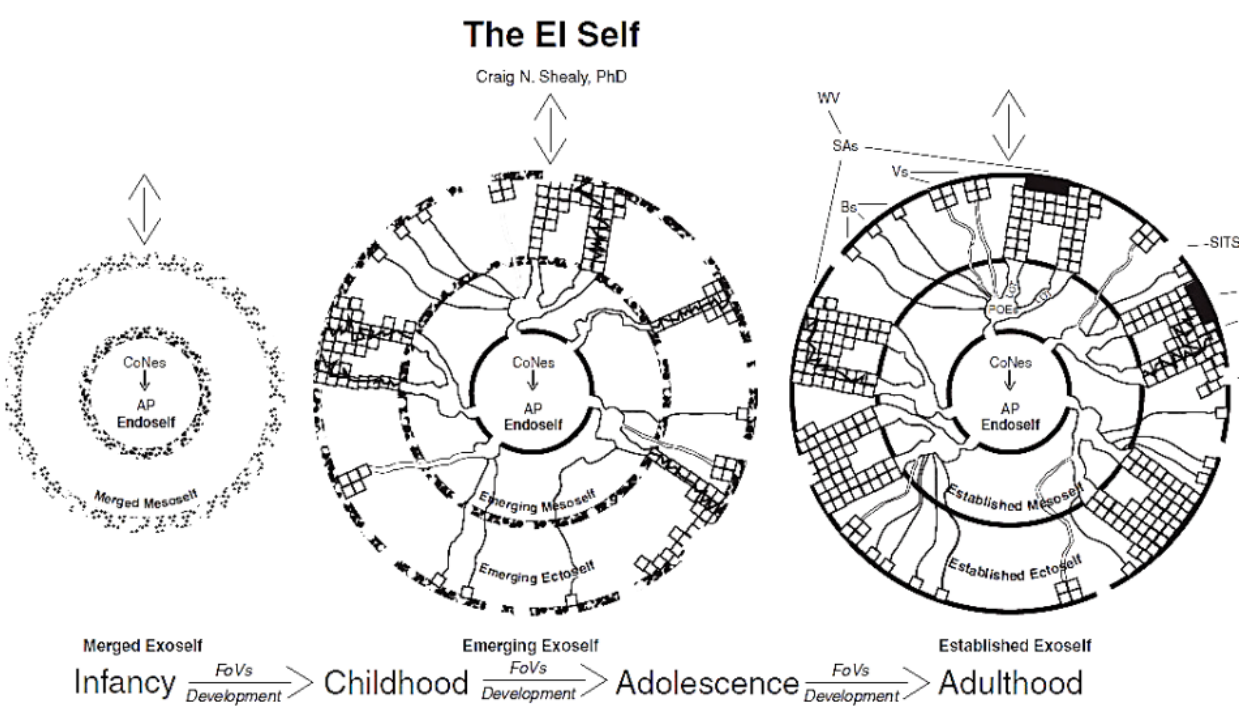

Figure 1. A pictographic model of the EI Self (Shealy, 2015).

While EI Theory is useful in bringing us to an understanding of how students may need or begin the intervention, it does not entirely offer a framework for the pedagogy necessary to elicit change - the catalyst. It is necessary, therefore, to employ Transformative Learning Theory (Mezirow, 1997) as a framework for how these experiences can be expected to produce changes in awareness of self and other as well as critical thinking. For the framew ork of this study, it is through transformative learning experiences, like GDL practices, that students become aware of their worldview and their ability to think critically about it.

Dalir found himself in conflict between his belief that people should not be discriminated against for characteristics of themselves they could not control and his need for affiliation with peers from his home country. Through the GDL experience, Dalir was able to share experiences and conversations with students from a spectrum of gender orientations that sparked reflection and created a new awareness of his value for family and the needs met by family. He also began to try to imagine how his family context would change, if one of his parents had "chosen" to change his or her sexual orientation. 
His interactions with the variety of participants provided an overwhelming amount of input from which he struggled to make meaning. Would he change his belief about sexual orientation being a choice? Was that a learning outcome considered by the faculty who designed the experience? Where was the cognitive space for the content they did intend him to learn?

\section{Transformative Learning Theory}

EI Theory provides the context for how Dalir formed the beliefs about himself and others that he brought to the learning experience. It also explains the interconnectedness of core needs and belief formation that can provide understandings of why learning or change is resisted at times. There is a gap, however, between knowing how a student's worldview is formed and the learning environment. Transformative Learning Theory (Hoggan, 2016) can provide the bridge between the two contexts by identifying components of the student that should change in order for the experience to be identified as high impact. This allows the educator to systematically design learning experiences and environments that could support change in each of these components. In the section that follows, a brief discussion of all of the components of Transformative Learning Theory are accompanied by an in-depth description of the components involved in this study.

All of the input Dalir received was providing input for a change in how he saw the world through the lens of his values, beliefs, and needs. Transformative Learning Theory is, "the process of effecting change in a frame of reference" (Mezirow, 1997, p. 5). In his Theory, Mezirow (1997) outlined the process involved in affecting change that, while critiqued and modified over the past two decades, has been foundational in the field of 
adult learning. Hoggan (2016) completed a meta-analysis of 206 studies that took place between 2003 and 2014 concerning transformative learning and developed a taxonomy to describe essential components of transformation which were present in the body of studies he reviewed. His work outlines six fundamental components: (a) a change in worldview, (b) changes in ways of knowing, (c) changes in cognitive ability, (d) increased awareness of self and other, (e) increased mindful awareness of subconscious dialogue, and (f) changes in action (Hoggan, 2016).

Using Hoggan's (2016) frame, a well-designed GDL practice should seek to address change in each of these six components of a transformative learning experience. For the scope and purposes of this study, three components will serve as dependent variables as operationalized by the Beliefs, Events, and Values Inventory (Shealy, 2004): (a) worldview change, operationalized by the Other Access domain of the BEVI; (b) increased self-awareness operationalized by the Self Access domain of the BEVI; and (c) changes in cognitive ability operationalized by the Critical Thinking domain of the BEVI. Changes in action, ways of knowing and subconscious dialogue are not captured in the available dataset but should be considered for future study.

In an effort to elucidate the relationships between the two theories, pedagogical approach, and assessment instrument used in this study, Table 1 provides across references for concepts central to EI Theory, Transformative Learning Theory, High Impact practice literature, and the component of the instrument used in this study, the Beliefs, Events, and Values Inventory (BEVI) created by Craig Shealy (2004). A discussion of the Transformative Learning components operationalized in this study follow Table 1. 
Table 1

Alignment of Theoretical Framework to Practice and Measures

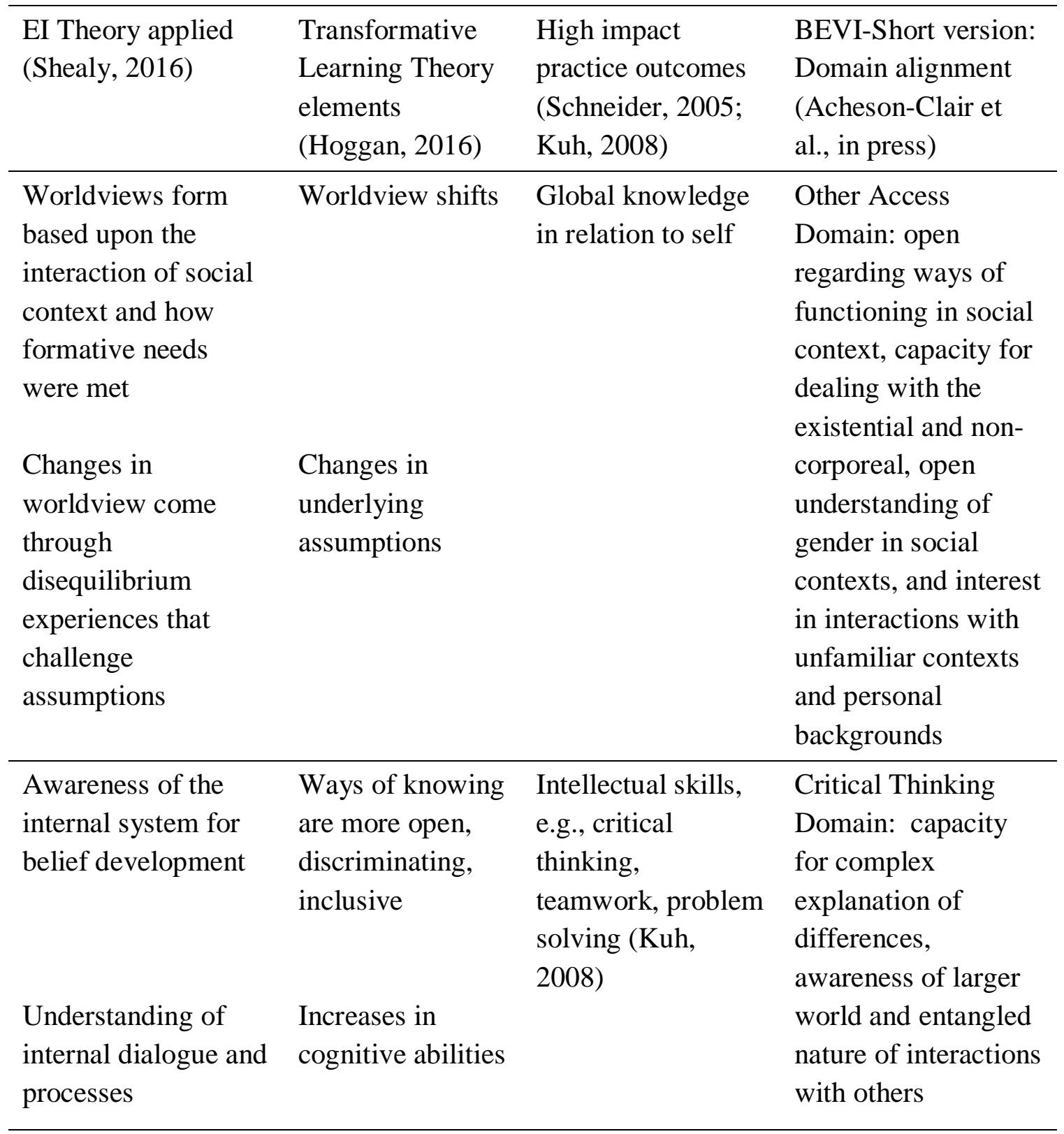

(continued) 
Table 1. continued

\begin{tabular}{|c|c|c|c|}
\hline $\begin{array}{l}\text { EI Theory applied } \\
\text { (Shealy, 2016) }\end{array}$ & $\begin{array}{l}\text { Transformative } \\
\text { Learning Theory } \\
\text { elements } \\
\text { (Hoggan, 2016) }\end{array}$ & $\begin{array}{l}\text { High impact } \\
\text { practice outcomes } \\
\text { (Kuh, 2008; } \\
\text { Schneider, 2005) }\end{array}$ & $\begin{array}{l}\text { BEVI-Short version: } \\
\text { Domain alignment } \\
\text { (Acheson-Clair et } \\
\text { al., in press) }\end{array}$ \\
\hline $\begin{array}{l}\text { To change, people } \\
\text { must have awareness } \\
\text { of core needs } \\
\text { (affective, } \\
\text { affiliative) and } \\
\text { personal beliefs }\end{array}$ & $\begin{array}{l}\text { Ways of being, } \\
\text { more emotionally } \\
\text { in tune, } \\
\text { independent and } \\
\text { aware }\end{array}$ & $\begin{array}{l}\text { Personal and Social } \\
\text { Responsibility, } \\
\text { e.g., intercultural } \\
\text { awareness, civic } \\
\text { knowledge, and } \\
\text { action (Kuh, 2008) }\end{array}$ & $\begin{array}{l}\text { Self Access Domain: } \\
\text { cares for human } \\
\text { condition, tolerates } \\
\text { difficult feelings, } \\
\text { resilient, } \\
\text { receptive to } \\
\text { corporeal needs, } \\
\text { sensitive, social, } \\
\text { values the } \\
\text { expression of affect }\end{array}$ \\
\hline $\begin{array}{l}\text { As awareness of } \\
\text { core needs and their } \\
\text { relationship to } \\
\text { beliefs becomes } \\
\text { apparent, people can } \\
\text { see themselves } \\
\text { independent of their } \\
\text { social context }\end{array}$ & $\begin{array}{l}\text { Changes in sense } \\
\text { of identity, } \\
\text { efficacy, } \\
\text { empowerment }\end{array}$ & $\begin{array}{l}\text { Personal and social } \\
\text { responsibility, e.g., } \\
\text { intercultural } \\
\text { awareness, civic } \\
\text { knowledge and } \\
\text { action (Kuh, 2008) }\end{array}$ & $\begin{array}{l}\text { Self Access Domain: } \\
\text { introspective; } \\
\text { accepts complexity } \\
\text { of self; cares for } \\
\text { human condition, } \\
\text { open regarding } \\
\text { practices in social } \\
\text { context }\end{array}$ \\
\hline & $\begin{array}{l}\text { Behavior aligns } \\
\text { with changes in } \\
\text { worldview } \\
\text { framework }\end{array}$ & $\begin{array}{l}\text { Applied learning, } \\
\text { civic engagement, } \\
\text { ethical action } \\
(2008)\end{array}$ & \\
\hline
\end{tabular}

Note. Modified from Acheson, et al. (In press).

Self. In Hoggan's (2016) meta-analysis of Transformative Learning studies an overarching theme of changes in the self emerged. He labeled this component simply, the Self. Hoggan found that many studies referred to a transformation in how the study participants saw themselves in relationship to the outside world due to some intervening process. There were subthemes of empowerment and increased responsibility as a result of changes in how study participants viewed themselves through their experiences. 
Another subtheme of the Self component came from studies that noted how participants became aware of or changed the purpose for their lives.

There is an expressed sense among some scholars of GDL practices that the purpose of GDL practices is to create a learning environment where students can gain a broader awareness of the world and, through that awareness, refine their understanding of how and through what systems they are connected to the world outside of the self (Agnew \& Kahn, 2014; Gorski, 2008; Kahn \& Agnew, 2017; Law, 2014; Milem, Chang, \& Antonio, 2005; Ogden, 2007; Whitehead, 2016). Mindful awareness of what educators are asking of students is warranted (Ettling, 2012). Through the insights provided by EI Theory, however, educators can be more mindful and informed about how to support students in a process that can often be disorienting if not painful.

Worldview. This component of Transformative Learning Theory is about the realm outside of the self. It encompasses all things that could be considered 'other,' not just individuals but social systems as well. Hoggan (2016) notes that many of the studies he reviewed captured changes in how participants made sense of the environment around them. He also noted that several studies included new awareness, not just that there were differences between the way they behaved and saw the world. Hoggan noted that participants were "becoming aware of the existence of social, economic, and political contradictions in society or the role power, privilege, and oppression play in people's lives" (2016, p. 66).

EI Theory provides educators with the framework for understanding how students differentiate between what they consider self and other. Transformative Learning Theory provides the bridge into pedagogical realms. For GDL practices to have lasting impact, 
they must be designed to help students develop new awareness of the world outside of the self. Practices should bring students into contact with their own values and mental narratives in service of helping them see that those systems serve as a filter for what comes from the outside world.

Epistemology and Capacity. These two components of Transformative Learning Theory are presented together because they fit into the EI Theory framework for critical thinking. Hoggan (2016) identified the theme of epistemology, or ways of knowing, in a number of studies he reviewed. Studies identified changes in how study participants defined knowledge or acquired knowledge. There were also findings that participants became more nuanced and critical about how they took in new information. Participants moved from passive and dualistic knowledge systems to active and nuanced systems.

Additionally, capacity for cognitive development emerged as a theme of research in Hoggan's (2016) meta-analysis. Studies noted changes in consciousness were also noted in this component. Transformative practices facilitated shifts from ego-centric to more global-centric orientations in study participants. Hoggan notes that increased ability to think with nuance and complexity allows learners to become more conscious of their self and its location within a wider social context.

Finally, it is important to take note of the entangled nature of processes within the self, like increases in critical thinking that give rise to increased awareness of one's social context. EI Theory and the model of the EI Self help make those systems within the learner clearer to educators designing complex GDL practices. Transformative Learning Theory can then help identify those components of the learner that can be transformed 
through learning experiences. The BEVI, founded on EI Theory, is a useful tool for operationalizing these elements of Transformative Learning Theory. The nested nature of the scales into larger domains mirrors the multi-component, highly interrelated nature of Transformative Learning Theory.

\section{Study Design}

Setting. The context for the study was higher education institutions and study abroad provider organizations across the US and overseas with a focus on GDL practices. These include education abroad programs for both US citizens and non-US citizens, global/international/multicultural living learning communities; global studies courses; and multicultural programming.

Participants. Archival data was used for the outcome variables of Self Access, Other Access and Critical Thinking. The dataset was composed of Beliefs, Events, and Values Inventory (Shealy, 2004) scores and background information from students who have participated in at least one type of GDL practice while during their course of undergraduate study. The participants were university students ages 17-28 who have voluntarily chosen to participate in a GDL practice. Their experiences had taken place between six months and seven years prior to this study. The institutions delivering the GDL practices range from large public institutions to small liberal arts universities and non-profit educational organizations.

Instruments. There was one instrument involved in data collection in this study. It is widely used by institutions of higher education around the world to collect data for program evaluation and research purposes. Each primary investigator whose data 
contributed to the database was required to receive BEVI training and proof of review from their own institution's IRB committee before collecting data.

The Beliefs Events and Values Inventory (BEVI). The BEVI, a set of psychometric scales developed by Craig Shealy (2004), was the data collection instrument utilized to capture data that will be used in this study. The BEVI is "designed to identify and predict a variety of developmental, affective, and attributional processes and outcomes that are integral to EI Theory...” (Shealy, 2004, p. 1075). The instrument's development began in the early 1990s and has undergone consistent review to maintain validity through Confirmatory Factor Analyses (Shealy, 2015).

There is a short and long version of the BEVI. The short version, used to collect all of the data in this study, contains 185 items. It includes the following components: (a) an extensive, modifiable set of demographic questions; (b) life history questions; (c) two validity scales; (d) seventeen psychometric scales; and (e) three qualitative items intended to collect participant reflections on their experiences. The short version is the primary version utilized by most institutions delivering GDL programs (Shealy, 2015).

Reliability and validity. Reliability coefficients for each of the scales have been recorded at above 0.80 and 0.90 (Shealy, 2015). According to Creswell (2012), levels of .80 and above are considered high reliability. Therefore, this is a strength of this instrument and an additional reason for its selection.

Researchers have indicated validity of the BEVI due to its ability to predict group membership across demographic variables. One study found that the BEVI was able to classify and predict group membership of mental health professionals and evangelical Christians (Hayes, 2001). In addition, Reisweber (2008) demonstrated the concurrent 
validity of the BEVI by predicting students who had increased their level of intercultural competence as measured by the Intercultural Development Inventory (Hammer, Bennett, \& Wiseman, 2003) and the BEVI. Validity is an important consideration for instrument selection (Creswell, 2012). Therefore, the validity and alignment with the study constructs were considered a strength of the instrument.

Scale and domain composition. Scores are calculated for each of the seventeen scales in the short version. Additionally, scales that are closely correlated are further organized under seven overarching domains for which composite scores are available. This study will use composite scores from three BEVI domains: (a) Self Access, (b) Other Access, and (c) Critical Thinking. These three domains, which represent eleven of the seventeen subscales, will be utilized as outcome variables for the purpose of this study. The composition of the domains and scales of the BEVI are included in Table 2 The domains in bold font will be used in this study. 
Table 2

Domains and Scales of the BEVI

\begin{tabular}{lc}
\hline \multicolumn{1}{c}{ Domain } & Description of scales within each domain \\
\hline Validity Scales & Consistency: captures consistency of response to differently \\
worded items that measure the same construct & Congruency: degree to which responses follow statistically \\
expected patterns & \\
Formative & Demographic: background items such as gender, economic \\
Variables & status, age, ethnicity, etc. \\
& Scale 1: Negative Life Events (conflict in family, trouble as \\
child, etc.)
\end{tabular}

(continued) 
Table 2 (continued)

Domain
Self Access
Scale 9: Physical Resonance: receptive to needs and feelings
of own body
Scale 10: Emotional Attunement: connected to own emotions;
sensitive to and accepting of expressions of affect in
others
Scale 11: Self Awareness: reflective, okay with complexity
and difficult feelings
Scale 12: Meaning Quest: seeking balance in life; searching
for meaning
Scale 13: Religions Traditionalism: sees life as mediated by
God, highly committed to religious doctrine
Scale 14: Gender Traditionalism: binary in thinking about
sexes and roles that are assigned to sexes. Prefers
simple view of sex and gender.
Scale 15: Sociocultural Openness: open to an array of policies
and practices; looks for experience of difference
Scale 16: Ecological Resonance: highly committed to
environmental sustainability
cultures, share experience with others from differing
culture groups

Note. Domains in bold are those examined in the study.

\section{Variables of Interest}

Focal, predictor variable: GDL practice types. The primary predictor variables of interest were types of GDL practices. All analyses focused on these variables to better understand their relationship to each other and their contribution to change in the outcome variables. Practices represented in the dataset were coded into one of four categories that correspond to definitions of GDL practices by Kuh (2008) and Kahn and 
Agnew (2017). These practices are (a) education abroad programs, (b) global studies courses, (c) global/international/multicultural living-learning communities, and (d) multicultural programming. All of these practices aim to increase students' awareness of their own worldviews through contact with beliefs different from their own, leading to reflection, critical thinking about the meaning of these differences. While practices vary in design within and between categories, they share these outcomes goals in the literature. In depth descriptions of these programs are listed in Table 1.3.

Table 3

Descriptions of Global and Diversity Practice Types

\begin{tabular}{cl}
\hline GDL Practice Type & \multicolumn{1}{c}{ Description } \\
\hline $\begin{array}{c}\text { Education Abroad } \\
\text { Program }\end{array}$ & $\begin{array}{l}\text { For the purpose of this study, these types of experiences } \\
\text { include US or international students traveling to a country } \\
\text { they do not consider their country of origin to study, complete } \\
\text { an internship, participation in service learning, or participate } \\
\text { in research. These experiences can range from one week to } \\
\text { one year in duration and may be facilitated by either their } \\
\text { home or host institution or a third-party provider of } \\
\text { experiences. }\end{array}$ \\
\hline $\begin{array}{c}\text { Multicultural } \\
\text { Community }\end{array}$ & $\begin{array}{l}\text { This type of practice involves students from differing } \\
\text { backgrounds living together and participating in coursework } \\
\text { or other programming that brings the group of students } \\
\text { together to interact. An example of this type of practice } \\
\text { would be residence halls that are designated as foreign } \\
\text { language learning spaces where a target language other than } \\
\text { English is spoken. Both students who are learning and native } \\
\text { speakers of the language live in the same hall. }\end{array}$ \\
\hline
\end{tabular}

(continued) 
Table 3 (continued)

GDL Practice Type

Description

This type of GDL practice is typically a credit-bearing course

that facilitates learning about global systems and social issues

Global Studies

Course

from a variety of places around the world. While this type of

course is typically part of a degree program for credit, it is not

the defining feature of the practice. The integrated content

connecting systems that impact multiple world regions is the

critical feature of global study practices.

This type of practice would include intergroup dialogue

Multicultural

programs, multicultural fairs or films, special lectures or

Programming

concerts targeting exposure to culture and traditions outside of

the participants' own culture and traditions.

Outcome Variables. This study examined relationships between the GDL

practice variables mentioned in the prior section with outcome variables operationalized

through the BEVI. As described in the instruments section, the BEVI is comprised of 17

scales (Shealy, 2015). The outcome variables described here represent multiple scales

per construct. Because of the intertwined nature of the constructs, factors from one scale

are related to other scales. A higher order construct name, "domain," was created for

each of these collections of scales. For each construct, a score ranging from 0-100 is

possible. Descriptions of key variables of interest are summarized in Table 4.

Self Access. A multi-faceted construct, Self Access, as measured by the Beliefs,

Events and Values Inventory, is the ability of individuals to recognize and make sense of their own emotions; and identify their beliefs and values, the origins of those values, and the implications for action indicated by those beliefs and values (Shealy, 2006).

Individuals who score in the upper 50th percentile are more likely to have the ability to 
understand their motivations in a wide array of contexts than individuals who score in the lower 50th percentile. Self Access is a larger construct composed of four scales with 1530 items per scale. The four scales that make up the Self Access domain of the BEVI are highly inter-related: (a) Emotional Attunement, (b) Physical Resonance, (c) SelfAwareness, and (d) Meaning Quest.

The first scale, Emotional Attunement, captures whether a person values the expression of affect and can express and perceive emotions. Example items are: 'I do not mind expressions of emotions' and 'Sometimes weakness can be a virtue' (Wandschneider et al., 2015). Participants respond with their level of agreement, but no neutral option is available. The Physical Resonance scale captures the level of attention people pay to their corporeal needs and how experiential they report being. An example item is: 'My body is very sensitive to what I feel.' The Self-Awareness scale captures peoples' ability to recognize and accept their own complexity and cope with difficult thoughts and feelings. Example items are: 'I have problems I need to work on' and 'I am always trying to understand myself better.' Finally, the Meaning Quest scale measures a person's desire for balance and meaning in life. An example item is: 'I think a lot about the meaning of life.'

Other Access. This construct is an individual's ability to identify the beliefs, values, and emotions of others. It is often accompanied by the ability to take on another's perspective. Additionally, it is important to note that Other Access also includes the capacity to cope with 'other' or 'different' socially constructed systems, e.g. different ways of educating, keeping social order, leadership, etc. Three scales with multiple items 
in each make up this construct: (a) Religious Traditionalism, (b) Gender Traditionalism, and (c) Sociocultural Openness.

The first scale, Religious Traditionalism, measures responses to how strongly a person believes that events and behaviors are mediated by a spiritual force. It also captures dualistic thinking about beliefs through statements like, 'There is one way to heaven.' Next, the Gender Traditionalism scale captures a person's orientation toward roles for genders - whether they prefer more traditional male and female roles or not. An example item is: 'Men are meant to be strong.' Finally, the Sociocultural Openness scale captures the degree of acceptance and readiness one has for experiencing and accepting new actions and policies in the realms of human structures and systems like education, religion, economics, or politics. An example item is: 'We should try to understand cultures that are different from our own.'

Critical Thinking. Related to perspective taking in the Other Access domain, critical thinking is the ability to be aware of the difference between one's own assumptions of the world versus the assumptions of others and assess the difference for validity and meaning (Merriam \& Bierema, 2014). The BEVI captures that ability along with another orientation toward simplistic thinking. The two scales that compose the Critical Thinking construct of the BEVI are Basic Determinism and Socioemotional Convergence. The Basic Determinism scale measures a person's tendency to prefer simple, dualistic explanations for behavior and differences. It also captures whether a person believes that change is possible. An example item from this scale is: 'It is only natural that the strong will survive.' The Socioemotional Convergence scale captures a person's ability to be aware of the beliefs and values of others as well as understand the 
complexity and nuance of beliefs. It juxtaposes items such as, 'We should do more to help those who are less fortunate' with statements like, 'Too many people do not meet their responsibilities.'

Prior research and theory indicate that there should be a connection between GDL practices and the outcomes of interest in this study. Shealy (2015) demonstrated that students increased their critical thinking scores on the BEVI after participating in study abroad experiences. Intergroup dialogue research has demonstrated that through participation in dialogue students are able to understand the beliefs and values of other students (Nagda, Gurin, Sorenson, \& Zúñiga, 2009). The Georgetown consortium report (Connor-Linton et al., 2009) and the Forum BEVI report (Wandschneider et al., 2015) both identify positive changes in self-awareness and other-awareness as a result of international study and multicultural initiatives on US campuses.

Finally, the operationalization of the construct of other-awareness has too often implied that the 'other' was in some way different in belief, behavior, or culture than the individual under observation. Scholars argue that this type of operationalization encourages stereotypes and focuses on difference rather than focusing on commonalities that could inspire unity (Woolf, 2013). The BEVI does not use specific groups as examples in any of its items. The BEVI instructions do suggest that participants consider their current program context as they respond to items.

\section{Variables: Student Characteristics}

Going back to the theoretical framework, Shealy (2015) points out that characteristics and formative backgrounds of learners should be kept in mind as administrators and faculty design the learning experience. Prior studies (Connor-Linton 
et al., 2009; Cindy Kilgo, Ezell-Sheets, \& Pascarella, 2015; Pike, Kuh, \& McCormick, 2011; Wandschneider et al., 2015) have demonstrated that characteristics such as gender, ethnicity, socio-economic status, and prior participation in GDL practices have explanatory value in student learning outcomes related to GDL practices. The following variables will be considered for their contribution to outcome gains on the four GDL practices analyzed in this study.

Socio-economic status. Cruse, Kinzie, Kuh, and Shoup (2008) found that several studies of GDL practices have demonstrated that socio-economic status (SES) contributes to students' decisions about engaging in global and diversity practices. Fewer low SES students participate in high impact GDL practices than peers in higher income brackets (Kuh, 2008). This variable is of interest in this study because it could increase understanding of relationships between different types of practices, SES groups, and changes in Self Access, Other Access and Critical Thinking.

Female. Soria and Troisi (2014) found that undergraduate females in their national study were more likely to self-report greater gains in global competencies (to include self-awareness) and participate in GDL practices in higher numbers than male peers. The dataset used in this study contains binary information regarding gender. It is still useful to understand interactions between genders and other variables to better understand what contributes to differential learning among each gender.

US Citizens. Because the population in this study is multi-national in nature, it will be useful to consider how US citizens differ from non-citizens as they participate in GDL programming. 
Table 4

Descriptions of Key Variables of Interest

\begin{tabular}{|c|c|c|}
\hline Variable Name & Type & Scale \\
\hline ID Number & Numeric & $1-2100$ \\
\hline \multirow[t]{6}{*}{ Type of GDL Practice } & Categorical & 1 = Education Abroad \\
\hline & & $2=$ Living-Learning Community \\
\hline & & with intercultural goals \\
\hline & & $3=$ Diversity $/$ multi-cultural \\
\hline & & programming \\
\hline & & $4=$ Global Studies Course \\
\hline \multirow[t]{11}{*}{ Socio-economic Status } & Numeric & $1=<\$ 10,000$ \\
\hline & & $2=\$ 20,000$ \\
\hline & & $3=\$ 40,000$ \\
\hline & & $4=\$ 60,000$ \\
\hline & & $5=\$ 80,000$ \\
\hline & & $6=\$ 100,000$ \\
\hline & & $7=\$ 120,000$ \\
\hline & & $8=\$ 140,000$ \\
\hline & & $9=\$ 162,000$ \\
\hline & & $10=\$ 187,000$ \\
\hline & & $11=>\$ 200,000$ \\
\hline \multirow[t]{2}{*}{ Female } & Binary & $0=$ male \\
\hline & & $1=$ female \\
\hline Age & Continuous & 16-65 years \\
\hline \multirow[t]{2}{*}{ US Citizens } & Binary & $0=\mathrm{No}$ \\
\hline & & $1=$ Yes \\
\hline
\end{tabular}


Table 4 (continued)

\begin{tabular}{|c|c|c|}
\hline Variable Name & Type & Scale \\
\hline Have been abroad ${ }^{\mathrm{a}}$ & Binary & $\begin{array}{l}0=\text { No } \\
1=\text { Yes }\end{array}$ \\
\hline $\begin{array}{l}\text { Have completed } \\
\text { multicultural coursework }{ }^{\mathrm{b}}\end{array}$ & Binary & $\begin{array}{l}0=\text { No } \\
1=\text { Yes }\end{array}$ \\
\hline Self Access & Numeric & -100 to 100 \\
\hline Other Access & Numeric & $-100^{c}$ to 100 \\
\hline Critical Thinking & Numeric & $-100^{c}$ to 100 \\
\hline \multicolumn{3}{|c|}{$\begin{array}{l}\text { 'Includes cases that selected any of the current variables: Service learning abroad, study } \\
\text { abroad, travel abroad, work abroad. } \\
\text { bIncludes cases that selected any of the current variables: Living with a roommate from } \\
\text { another country or different ethnic background, Participation in a course with an } \\
\text { international or multicultural focus, Participation in on-campus international or } \\
\text { multicultural events, Studying a language other than English, Travel to five or more } \\
\text { states in the United States, Living in a residence that is international, multicultural and/or } \\
\text { language-focused, Participating in off-campus international or multicultural events. } \\
\text { 'Negative indicates that the pre-experience score was greater than the post-experience } \\
\text { score. }\end{array}$} \\
\hline
\end{tabular}

Have Been Abroad. Wandschneider et al. (2015) reported that students who have been abroad before showing greater interest in exploring differences and repeating international study. It is important to explore this variable as a moderator for the relationship between program type and changes in Self Access, Other Access and Critical Thinking.

Have Completed Multicultural Coursework. In the same way that education abroad attracts students who are already predisposed to exploring self and other, students who opt into multicultural coursew ork also tend to be looking for an experience of different ways of being or knowing (Wandschneider et al., 2015). 


\section{Data Analysis}

Prior research on GDL practices has been done in isolation, focusing on one practice type or learning outcome at a time instead of comparing the impact different practice types in relation to multiple learning outcomes. Additionally, the empirical evidence to support claims for the impact of a single practice type, like education abroad, is based upon research that is subject to limitations of small sample size and over reliance on student self-report of learning (Ogden, 2015).

Using SPSS, I completed two types of analysis. The first analysis was two, oneway analysis of covariance (ANCOVA) models. The second was six multiple regression analysis models with target variables of GDL practice types and outcome variables to understand to what extent a relationship exists between practice types and Self Access, Other Access, and Critical Thinking outcomes.

The two ANCOVA models allowed me to identify relationship between practice types and each individual outcome. I used a covariate of starting score to allow me to take into consideration differential starting points for participants. For example, if strong critical thinkers were preferentially drawn to global studies instead of living-learning communities, I needed to account for that in order to avoid misattribution of the change scores to participation in global studies. This method complements the IE framework to highlight how students will enter experiences at different levels of readiness and those levels of readiness may impact how they experience the practice and learn.

For the regression analysis, four models with seven comparisons each will be analyzed to understand the relationship between changes in Self Access, Other Access, Critical Thinking; Program Type and Duration; and Participant Characteristics. Each 
model will be focused on one of the three outcome variables. An example of the Self Access model appears in Figure 2.

Using SPSS with BEVI outcomes data, I completed a multiple regression analysis in three stages to explore the relationship between practice type as the target independent variable and gain scores on three outcomes: (a) Self Access, (b) Other Access, and (c) Critical Thinking. The first stage was a simple regression using dummy variables to represent each of the four practice types. For each program represented in the BEVI data, a primary practice type was indicated. The regression coefficient calculated for each of the practice types was an indicator of the relationship between the presence of that practice and positive changes in the target outcome.

The second stage of the analysis incorporated a set of variables. These included both demographic student traits, such as gender and ethnicity, as well as program characteristics, such as duration. Inclusion of a control of Time 1 scores increased the accuracy of the analysis, as this variable may have non-negligible relationships with practice types. Controlling for Time 1 score influence reduced spurious results coming from misattribution based on different starting points, giving a stronger answer to the first research question.

The final stage of the analysis added first level interactions between participant demographics and practice type to explore the possibility that a practice type may have differential impact on different subpopulations or under varying practice formats. Significant regression coefficients on an interaction term indicated that the practice type 
in question (e.g., service learning) had a meaningfully different impact on students with different traits (e.g., domestic versus international).

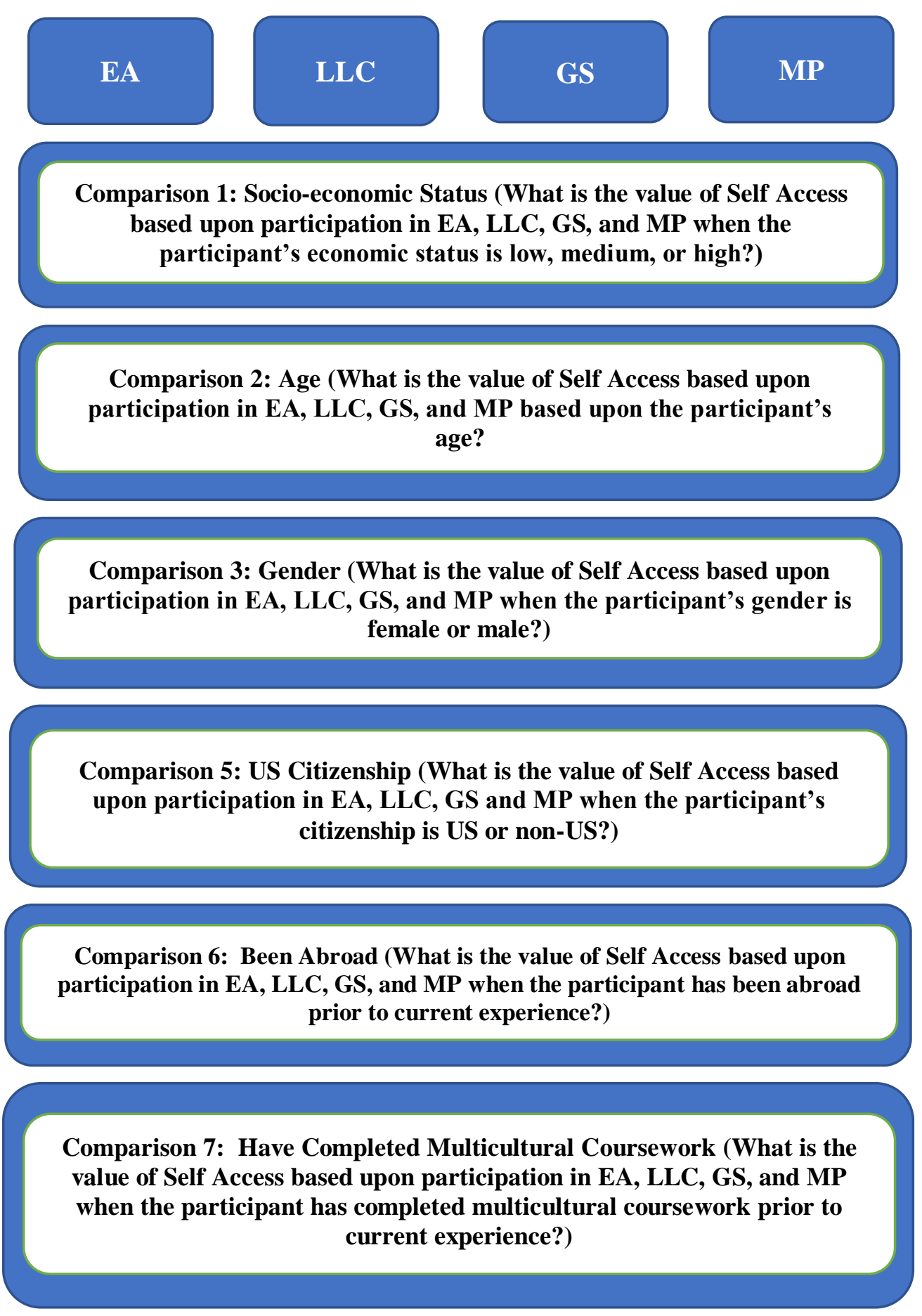

Figure 2. Self Access regression model example 
Ethical considerations. This non-experimental study used archival data from the Beliefs, Events, and Values Inventory (BEVI; Shealy, 2004) database. Data was collected through IRB-approved research projects within the past seven years. Because this study involved human subjects, protection of participant confidentiality was necessary. Participant student identification numbers and email addresses were not included in the archival dataset. All participant cases were coded with internal identification numbers so that they were anonymous. Terms and conditions for usage of the BEVI dataset were based upon an agreement that raw data will be stored securely offline, not shared with anyone outside the study, and only published in aggregate.

\section{Limitations}

One of the limitations of this study was the lack of random selection in the dataset. The nature (cost, time, degree requirements) of the experiences means that participants generally self-select into these GDL practices. It was, therefore, not possible to generalize findings beyond this dataset. Another limitation of the study was the lack of a control or comparison group. As with other important educational endeavors where it is not possible to deny or assign an experience, it was necessary to use creative methodologies such as propensity score matching, for example, that will allow researchers to understand the experience more deeply. In this case, the research interest was in understanding the differences between practice types not the difference between students who participated and those who did not.

The BEVI measures limited the study in some ways as well. The level of granularity on student characteristics was limited by how the items were coded and the 
wide array of participants who have taken the BEVI. Race, for example, was captured in nine categories. There were not enough participants in each category to meaningfully apply statistical functions, therefore, categories would have been combined, thus losing the ability to understand how programs might have different impacts on each of the nine categories. Additionally, the race categories would only be meaningful for the US citizens. Therefore, this variable was not used in the analysis. There are a large number of non-US citizens who are in this dataset because they are attending US institutions, however, there is no way to identify the country of origin for each participant. The categories are US and non-US, making further analysis impossible. Finally, duration could not be captured in this study because over half of the sample population did not respond to this item on the BEVI. Alternative options were considered, for example, utilizing the pre- and post-experience BEVI administration date stamps to calculate the number of days. This approach was unsuitable because individual protocols used to collect the data were not the same. Some institutions collected the BEVI data immediately before and after the GDL experience while others gathered data before but waited until six months post experience to collect Time 2 data.

In addition to the data-coding limitations, the interpretation of the analysis must consider all associations as the design does not permit causal inference. While there are more sophisticated techniques that would provide stronger causal inference from the data, the research questions are limited to associations between variables.

\section{Definitions and Key Terms}

For clarity, the following section provides the reader with definitions and key terms that are frequently used in the study. 
High impact practice (HIP). This is the broad umbrella of interventions intended to precipitate 21 st century learning outcomes. The terminology was developed during the Liberal Education and America's Promise campaign implemented by AAC\&U beginning in 2005 (Association of American Colleges \& Universities, 2011). AAC\&U identified ten categories of HIP that seek to facilitate change in how the student sees the world, e.g., increased intercultural awareness, enhanced self-awareness, improved critical thinking skills (Engle \& Engle, 2004; Kuh, 2008; Wandschneider et al., 2015). Global and diversity learning practices are one of the ten HIP categories that were the focus of this study.

These practices represent two distinct historical movements, global or international learning and diversity or multicultural learning, within US higher education that have often been adversarial. Those histories will be covered in more depth in Section 2 of this document. It is important to understand that each of the types of GDL practices often reside in separate units with distinct objectives in their university settings.

Global and diversity learning (GDL) practices. Nested within HIPs, GDL practices represent a large array of programs and experiences. There is currently no agreement among scholars or practitioners regarding a common definition. The AAC\&U used Hovland's (2014) definition and rubric in order to provide some consistency and some models for implementation and assessment. The term global was an intentional choice to include a focus on sociocultural contexts that is not restricted by national boundaries. It also allows for learning outcomes that focus on the entangled interactions of multi-layered local contexts. Scholars tend to agree that it is most important for staff, faculty, and students on a given campus to have a shared understanding of the term 
(Green, 2013; Kahn \& Agnew, 2017; Sandeen, 2012; Whitehead, 2015) whether or not there is a scholarly consensus for the term. Diversity learning programs have historically focused on engaging differences and seeking social justice for marginalized groups in the domestic context. The types of GDL practices involved in this study are described in the following paragraphs.

Education abroad. This practice of sending students from the US to study in universities outside of the US has been in place for more than a century (Hoffa, 2007). It became popularized after World War II as a means to support growing area studies programs, and was seen as a tool for diplomacy and regional influence during the cold war (Cornwell \& Stoddard, 1999; William Hoffa, 2007; Lou, Paige, \& Vande Berg, 2012). Typically practiced by affluent students of the dominant culture, education abroad participation has expanded somewhat in the past two decades (Connor-Linton et al., 2009; Lou et al., 2012; Tillman, 2011). Existing research, however, is limited due to funding and complexity of experience. Of the studies that do exist, many are limited in their scope due to selection bias, complexity of constructs, or scale (Ogden, 2015; Wandschneider et al., 2015) From foundational studies in the field, scholars have attributed outcomes like increases in self-awareness and other-awareness, increased foreign language skills, increased ability to communicate across cultural differences, and increased creativity—all skills targeted by LEAP (Engle \& Engle, 2004; Galinsky \& Maddux, 2009; Lou et al., 2012,Twombly, Salisbury, Tumanut, \& Klute, 2012;). Multicultural programming and coursework. This type of programming can be embedded within a degree program, such as a common course requirement for preservice teachers on the multicultural environment of US classrooms. Programming can 
also be extra-curricular like inter-group dialogue programs that address challenging issues such as race or gender relations - most commonly in the US context.

Global living-learning communities. While this practice always involves shared living space, the instructional format has many structural iterations (Kuh, 2008). They can take the shape of courses that are linked together in succession where a cohort of students shares an extended period of study with a common theme, such as international, multicultural, or language-focused (Pike, 2008; Pike et al., 2011). Another format that became popular in the late 1990s and 2000s is to have a cohort of freshman from a given institution begin their program of study in a community abroad.

International service learning programs. These types of programs seek to integrate course instruction and community service to provide different learning contexts as well as encourage civic learning. They commonly take place during university breaks in coursework. They can be credit bearing or non-credit bearing. It is also common for this type of learning to be embedded within a course.

Expected outcomes from global and diversity learning practices. While the previously listed practices vary in delivery, context, and content, they share a set of outcome goals. Universities look to these types of practices to bring interpersonal and cross-cultural content and skill development alongside professional content coursework. A number of studies link GDL practices to the development of 21 st century skills (Connor-Linton et al., 2009; Intolubbe-Chmil, Spreen, \& Swap, 2012; Nagda et al., 2009; Sutton \& Rubin, 2004; Wandschneider et al., 2015). Multiple studies have identified increases in self and other awareness and critical thinking, however, they have not considered how different practices may impact groups of students differentially. For 
example, education abroad may be more effective at producing other awareness in students than multicultural practices for some subsets of students. Studies like the Georgetown Consortium report (Connor-Linton et al., 2009) hint at the potential for some students to be put off by education abroad experiences and, when not well supported, driven to reinforce stereotypes students brought with them about the host community. This study seeks to identify whether there might be a relationship between practice attributes and student characteristics in this sample that might be instructive for practitioners as they design new experiences.

\section{Significance of the Study}

This study has implications for both scholarship and practice in US higher education. It is a necessary step to broaden understanding of how and why change does or does not occur and for whom when students participate in GDL practices (Ogden, 2015).

This study is the first step in understanding how practice type variables impact desired student learning outcomes of Self Access, Other Access, and Critical Thinking. Current studies which address outcomes of GDL practices focus on practices in isolation (Acheson-Clair et al., In press). Administrators in US higher education could benefit from an enhanced understanding of how types of GDL practices differ in their contributions to change (Wandschneider et al., 2015). It could allow for better complementarity of GDL offerings and coursework. It would also move scholars toward the next step to understand how GDL practices facilitate change.

The significance of this study for practitioners rests in the exploration of the interactions between types of practices and student characteristics. Knowing whether or 
not there are types of practices that are more effective for particular groups of students (not just from a demographic perspective) would permit program advisors and developers to counsel students more effectively or prepare students for experiences more accurately. It could also permit leaders of institutions to target a single student learning outcome more effectively. For example, universities that identify development of students who think critically as an element of their mission may benefit from understanding whether certain types of GDLs may more effectively contribute to that outcome versus other GDL types.

\section{Summary}

Anna, a study abroad student who was not sure she wanted to go home, encountered an experience that led her to question her own identity and challenged her to thinking critically about what her time abroad would mean when she returned home. While one can see the changes in her BEVI scale scores from the beginning of her semester abroad to the end, one does not entirely know whether this type of program was integral to bringing Anna to the space where she could begin to see herself and others through a new lens. Being able to understand whether this sort of change can occur for students similar to Anna who cannot study abroad could allow university leadership to make informed decisions about which types of high impact practices need funding and development for the types of students at their institutions.

This study involves the operationalization of four such practice types: (a) education abroad programs for both US citizens and non-US citizens, (b) global/international/multicultural living-learning communities, (c) global studies courses, and (d) multicultural programming. I used changes in scores on Self Access, Other 
Access, and Critical Thinking from the Beliefs, Events and Values Inventory (Shealy, 2004) as outcome variables to run a regression analysis with practice variables in order to understand the relationships between GDL practice type and student learning as measured by the BEVI. I also looked for relationships between student characteristics and practice types. Analysis of the results was then connected to the problem of practice and its context.

The value of this study is the potential use for GDL practice designers and administrators in US higher education. Knowing whether there is a meaningful connection between practice type and the changes we see in student awareness and critical thinking could increase effectiveness of staff advising, allow students to more accurately select programs that fit their goals, or permit institutional leadership to focus on the development of GDL practices that are more effective at producing a specific desired student learning outcome. Through understanding which types of practices are most effective at producing the types of changes in learning we see in Anna, we could then begin to explore why and how those practices work. Understanding how GDL practices work will allow institutions to better support students like Anna. Instead of leaving her to transition back to her home community, heavy with the weight of awareness of herself and those around her, faculty and staff responsible for GDL practices could design ways to support meaning making through the struggle to think critically and increase awareness. 


\section{SECTION TWO}

PRACTITIONER SETTING FOR THE STUDY 


\section{Practitioner Setting}

Nearly thirty years ago, Prahalad (1990) wrote a cautionary article on dangers of the growing gap between practitioners and researchers that applies to many disciplines. He noted that without collaboration between practitioners and researchers, institutions and their leaders would struggle “...in a world where variety, complex interaction patterns among various subunits, host governments, and customers, pressures of change and stability, and the need to re-assert individual identity in a complex web of organizational relationships are the norm" (p. 30).

Complexity in higher education organizational relationships has become the norm, and the consequences of the research-to-practice gap can be seen clearly in the growing field of global and diversity learning (GDL) practices, just one part of the nationwide push in US higher education to embed high impact practices (HIP) that purport to produce 21 st century skills. Reduced funding at the federal, and often state, levels combined with new immigration patterns and changes in political contexts leave university leaders with the responsibility to graduate students who can live and work in dynamic, complex environments while maintaining a sense open security in their

personal identity (Agnew, 2012; Williams, Berger, \& McClendon, 2005). However, the structures, systems of values, and beliefs and the political landscape of many US universities have not allowed practice to inform research or for research to inform practice in burgeoning fields of study within their own walls (Williams et al., 2005). This complexity of relationships leaves university leaders struggling to produce the new outcomes demanded by industry and civil society outside of their university's core function of conferring degrees based upon discipline specific content knowledge. In 
order to transform their institutions, leaders must find ways to inspire a new vision of their institution that brings self-awareness and other-awareness development into the core process of the organization.

The core process of universities has been based upon faculty members with expertise in specific content areas who relate that content to students, separate from other disciplines or external inputs (Friedman et al., 2015). The 21 st century outcomes that point to awareness of self and other not only represent changes in expectations for learning in US higher education but reflect changes in which institutional structures produce those outcomes. In the professional bureaucracy, aimed at standardization, skills and understandings are traditionally developed in lecture halls or labs with the faculty, the operating core (Mintzberg, 2005).

Advances in technology and greater global entanglement have increased the need for university graduates with knowledge of human cultures, critical thinking skills, the ability to work on a team, 'glocal' engagement (both global and local), ethical reasoning and action, and the ability to apply knowledge and skills developed in one learning context to different context (Brownell \& Swaner, 2010; Hart Research Associates, 2008; Kuh, 2008). Surveys conducted by higher education associations and government organizations have consistently found that employers and civic organizations prioritize skills and abilities like critical thinking and awareness of self and other over content knowledge and express concern that current graduates are not well prepared in these areas (Cornwell \& Stoddard, 1999; Hart Research Associates, 2008, 2016). Many faculty members see the boundary of their responsibilities and training in increasing student content knowledge and discipline-related skill development. If the facilitators of the core 
function of the institution believe this is their boundary, how will universities play a role in a building a society where people can be successful in understanding complexity or unthreatened and secure in their interactions with difference and change? How will students learn to work across differences?

Practitioners who have become researchers suggest that the outcomes industry and society desire are all predictable outcomes of high impact GDL practices (Kuh, 2005; Kuh. et al., 2015; Kuh \& O’Donnell, 2013; Lou et al., 2012). Studying abroad, taking a course on global studies, multicultural dialogue programs, and living-learning communities are all considered potential vectors for outcomes like critical thinking, and awareness of self and other. How can such a wide array of practices promise the same outcomes? Can a student who participates in an intergroup dialogue program on race be expected to gain knowledge of human culture in the same way or depth as a student who spends a year studying abroad in a context very different from her home culture? This is where the gap between practitioners and researchers becomes evident.

Understanding the historical development and current settings of GDL practices is useful to understanding the purpose of the study. This chapter outlines the history of the path that led universities to work around its operating core of faculty members. It also contains a brief analysis of the stakeholders in US higher education involved in GDL practices. Structural and political frames developed by Bolman and Deal (2013) as well as a model of learning organizations by Senge (2006) are used to provide a clearer understanding of complex organizations like universities. An explanation of the role of leadership using Transformational Leadership Theory follows the analysis of US higher 
education. The chapter culminates with a brief discussion of the implications of research for the practitioner in the GDL setting.

\section{History of GDLs in US Higher Education}

GDL practices represent a diverse collection of programs and initiatives in higher education with very different histories that have only very recently begun to overlap and collaborate. In the following sections, brief histories of global learning and diversity learning are outlined. The section concludes with discussion of recent efforts to bring global and diversity learning efforts together under a single banner.

Global learning practices. These types of practices vary widely from efforts to imbue the curriculum with perspectives from a variety of cultures to studying abroad to providing opportunities for domestic students to connect with different perspectives first hand by increasing international student enrollment. Hovland (2014) points out that there is no agreement among scholars or practitioners regarding a common definition, but he suggests in many definitions the word global was an intentional choice to include focus on sociocultural contexts that are not restricted by national boundaries. It also allows for learning outcomes that focus on the entangled interactions of multi-layered local contexts. A simple definition by Olson, Green, and Hill (2006) denotes this openness to complexity stating that global learning represents the aptitudes students "acquire through a variety of experiences that enable them to understand world cultures and events; analyze global systems; appreciate cultural differences; and apply this knowledge and appreciation to their lives as citizens and workers" (p. v). 
Global learning on an institutional level has also been known as internationalization which, some scholars argue, has inadvertently shifted the focus from diversity to national identity and culture (Hudzik, 2011).

Internationalization efforts first came out of multinational desires for peace after World War II (Bu, 1999; Hoffa, 2007; Twombly et al., 2012). At that time, study abroad programs were developed to bolster language skills that were needed for regional and area studies programs. Because US institutions did not have language study capacities at that time, students' options were largely limited to direct enrollment in a foreign university (Cornwell \& Stoddard, 1999; Hoffa, 2007). International exchanges were developed to promote common understanding between people in different nation-states. The focus of internationalization was rooted in foreign policy agenda, therefore, international programming developed a sense of prestige and within the context of the core function of the university (Olson et al., 2006).

Internationalization through student exchange has also had uneven distribution of participation historically. While participation in study abroad increased steadily with tertiary student enrollments from the 1950 s to the present day, international student enrollments increased exponentially over the same period. The past two decades have seen dramatic increases in international student enrollments for degree-seeking students (Institute of International Education, 2016). Between 1989 and 2015 there was an increase from nearly 400,000 to over 1 million international students coming to the US on exchange or seeking a degree. For the same time period, there was an increase from roughly 75,000 to just over 300,000 US students going abroad for on credit-bearing programs (Institute of International Education, 2016). What were once practices that met 
US foreign policy aims became revenue-generating practices for many institutions, particularly as state and federal governments began to dramatically reduce their support for higher education (Agnew \& VanBalkom, 2009; Knight \& Altbach, 2016). Initiatives to increase internationalization through increasing foreign student enrollment at many institutions was perceived by faculty as rhetoric to support revenue generation. Diversity offices also took issue with this practice as administrators counted international students in order to meet their diversity enrollment targets in lieu of increasing efforts to recruit and maintain domestic diversity (Cornwell \& Stoddard, 1999). This point will emerge again later in the chapter's discussion of the historic divide between global and diversity learning initiatives.

The shift away from foreign policy aims ultimately led global learning efforts toward diversity learning initiatives. A new focus on developing capacities in students for a diverse work place brought international and global studies programs to a value for interpersonal outcomes like awareness of self and other and critical thinking; each correspond to one of three large domains of 21 st century competencies outlined by the National Academy of Sciences (Pellegrino \& Hilton, 2012). These are competencies that the Academy deemed necessary not only for the successful professional life of all people but the personal life as well. The call to increase interpersonal learning and critical thinking outcomes did not become strategically targeted goals, however, until the 1990s (Cornwell \& Stoddard, 1999). According to Connor-Linton, Paige, and VandeBerg (2009), the shift occurred in response to the student-centered learning movement that occurred in the US which would ultimately lead to greater emphasis on formative and summative assessment of learning. 
Before the shift in focus, practitioners of global learning programs had been valued for their administrative skills. Typical study abroad or international student advisors were hired based upon their knowledge of immigration processes, foreign education systems, and internal workings of higher education. Therefore, the capacity to assess such complex experiences was not present in most international or global learning offices. Recognizing this capacity gap, early leaders sought to establish a research agenda for global learning, however, due to the content-specific nature of academia, finding scholars who had the interest or background knowledge to conduct research was challenging (Ogden, 2015). Designated funding for research in global learning was scant, and faculty members outside of area studies programs did not see global learning as a discipline of study. Around the middle of the 1990s, two peer-reviewed journals for global and intercultural learning were established to promote research and assessment (Connor-Linton et al., 2009). The scholar-practitioner gap persists, however, due to structural issues in higher education that will be discussed in the following sections.

Diversity Learning Practices. Diversity learning encompasses an even wider array of practices and, like global learning, also does not have a commonly held definition among scholars and practitioners (Sleeter \& Grant, 1987). Diversity learning practices range from curriculum integration of diverse perspectives, advocacy for restructuring education to provide access and equity, programs to promote awareness of diverse communities on campus, and efforts to support students in minority groups establish belonging and navigate university life. Ibarra (n.d.) notes how research in diversity can be understood in three dimensions: (a) structural, (b) multicultural, and (c) context. Structural diversity encompasses practices that seek to increase the numbers of 
diverse faculty and students at an institution. Multicultural diversity practices are those targeting increased awareness and acceptance of diverse cultures and perspectives. Context diversity practices target change in the way educational practices are viewed in order to address the need of all populations of students. This section deals with multicultural and context diversity facets as those are the arenas represented in the dataset used for the study.

In the context of US higher education, diversity learning and multicultural education practices began with the civil rights movements in the 1960s and 70s (Banks \& Banks, 1995; Gurin, Dey, Hurtado, \& Gurin, 2002a). In the 1970s, as universities sought to diversify their student bodies, academic programs in African American, gender, gay and lesbian, and Hispanic programs of study emerged (Olson, Evans, \& Shoenberg, 2007). Additionally, the multicultural education movement that began at the same time focused on preparing pre-professional students, like teachers, for the multicultural classrooms and communities in which they would live and work (Banks \& Banks, 1995). These practices did not initially receive the same industry or government support that global learning practices received as they were developed within higher education.

Students enrolled in diversity learning courses or programs disproportionately came from the groups that are the focus of the learning objectives. For some portion of these students, there was, and is, a desire to understand a broader context connected with one facet of their identity. Other students were, and are, motivated to participate through a desire to be heard or represented at the institution (Olson et al., 2007). In addition to identity study content areas, multicultural affairs offices developed to help support the emotional and social well-being of historically marginalized groups. These offices have 
traditionally been housed within student affairs arms of universities, operated by staff members with faculty supervisors or sponsors.

Gurin et al (2002b) pointed out how educators have historically been, and continue to be, pressured to demonstrate tangible connections between campus diversity initiatives and institutional learning outcomes. Therefore, it was imperative for researchers to understand and demonstrate outcomes of diversity learning early in their inception (Agnew, 2012; Cornwell \& Stoddard, 1999; Olson et al., 2007). To that end, research on the outcomes of many diversity learning practices is more prevalent than global learning, despite the much shorter history most of its practices have as part of higher education. The number of conceptualizations of diversity learning, however, make it difficult for the practitioner to track and apply.

With the end of the Cold War, leaders in global learning practices like education abroad began to focus more on self-awareness, other-awareness, and critical thinking skills (Hoffa \& DePaul, 2010). Scholars in related fields like intercultural communication began to see how diversity and global learning shared outcome goals (Bennett \& Bennett, 1994). It was difficult to align these practices, however, due to differences in philosophies, frameworks, funding, university structures, and participants.

\section{Organizational Analysis}

The goal of this study is to provide greater clarity regarding how, why, and for whom different types of GDL practices facilitate the development of critical thinking skills and awareness of self and other. Greater clarity will allow practitioners and leadership in higher education to allocate resources more effectively and communicate more efficiently with students about the benefits of participation. A brief look at the 
organizational context of GDL practices shows structural and political challenges that separate support staff from the expertise they need to evaluate efforts, leave staff struggling to gain faculty buy in due to skepticism and resource scarcity, and promote the status quo through assumed value of GDL practices.

GDL practices included in this study (living-learning communities, global studies courses, education abroad, and multicultural programing) are tangential to the academic core process. The model of the professional bureaucracy put forward by Mintzberg (2005) is an instructive tool for the investigation of how GDLs relate to other processes within in university. The model contains five parts: (a) the strategic apex, (b) middle line management, (c) a technosphere, (d) support staff, and (e) the operating core. For the US university, the apex represents governing boards and presidents, middle line management represents deans and provosts, the techno-structure contains assessment and compliance specialists and technology administrators, support staff are professionals who run programs like study abroad or intergroup dialogue as well as a wide array of human resource specialists and account managers, and the central operating core tasked with the primary function of the organization are faculty members. This structural reality isolates GDL practice administrators in a tower where they are challenged to access necessary student information, set up and conduct assessments of their programs, and communicate any strategic outcome information to the operating core of the university. 


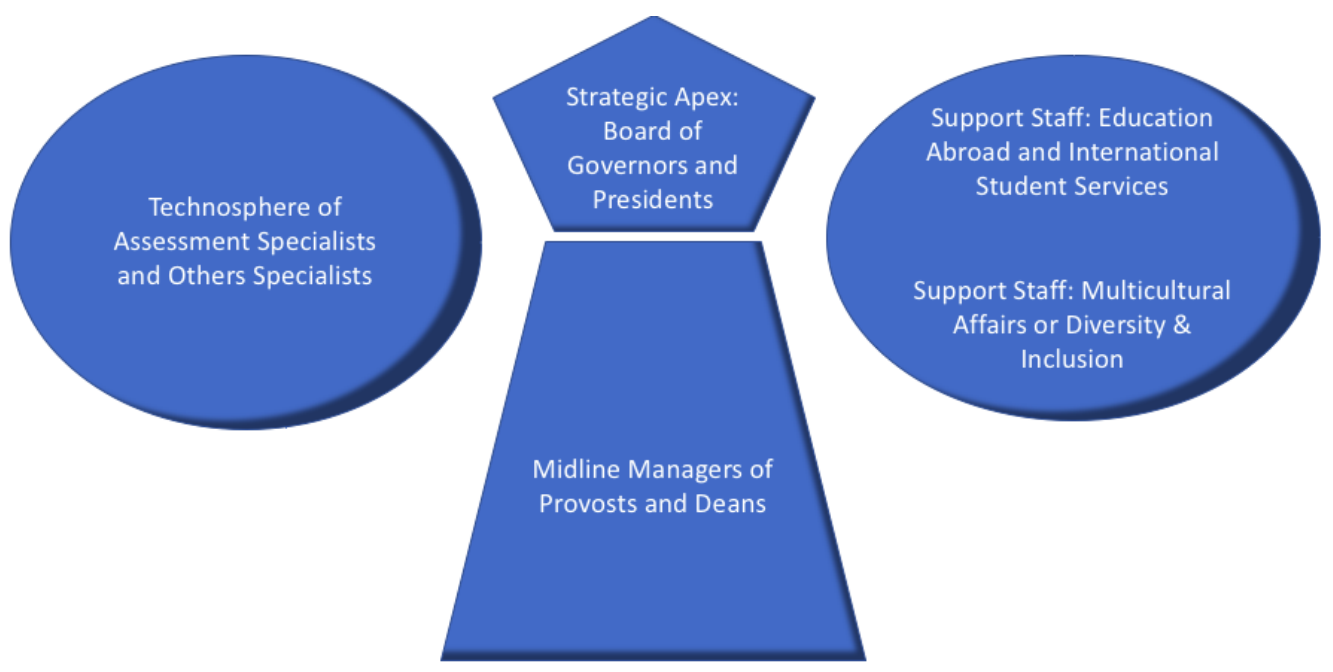

Traditional Operating Core of Faculty Members: Content-Specific Degree Programs and Research Outputs

Figure 3. Mintzberg's Organizational Model in the Context of GDL Practices

The model in Figure 3, uses Mintzberg's (2005) organizational model to demonstrate how support staff in GDL practice offices are isolated from the core functions of the institution. When viewed through Bolman and Deal's (2013) lenses, the model makes the structural and political context of GDL practices even clearer. The structural frame highlights the assessment challenges that can lead to legitimacy issues GDLs face regarding how they are perceived to align with the goals of the institution. The political frame allows the researcher to understand why certain types of GDL practices are privileged over others despite the lack of outcomes evidence. The political frame also provides a window into resource distribution and competition for resources between units that facilitate different GDL practices.

Going back to the theoretical framework for this study, who a student is prior to participation in a GDL practice will to some extent predict the interaction between the student and the practice itself and, ultimately, shape the transformative outcomes of the 
experience (Shealy, 2015; Wandschneider et al., 2015). Units within the university charged with facilitating GDL practices like education abroad, living-learning communities, and multicultural programming are part of the support staff or a collaboration in the case of living-learning communities. Generally, reports concerning student body demographics, pre-enrollment academic capacities, or information regarding prior experiences with programs that target awareness of self and other are distributed to midline management and the faculty in the operating core by institutional assessment groups in the technosphere. National Survey on Student Engagement (NSSE) data, for example, has proven useful for education abroad and multicultural programming professionals hoping to understand which engagement experiences students value (Kilgo, Ezell-Sheets, \& Pascarella, 2015). That data, however, is collected, reported and reviewed by staff in the technosphere and decisions based upon that information are made by midline dean or apex-level presidents. Sharing that type of data could provide benefits to support staff building and delivering GDL practices.

In addition to being outside of the distribution stream of student characteristic data, many GDL practice offices now lack the skills needed to assess experiences they design and facilitate. Administrators in education abroad, multicultural affairs, and international student services have not traditionally been hired for knowledge of theoretical frameworks, educational assessment strategies, or data collection skills (Ogden \& Streitwieser, 2016). Remembering the historical development of these practices, administrators served as support for academic units in which they were once embedded like area or identity studies programs (Connell, 2016; Cornwell \& Stoddard, 1999). Their role was to provide coordination support, e.g., visa applications, event 
planning, program marketing, and communication services, for activities that were supplemental to the course of study. As enrollments grew and universities reorganized, GDL practice administrators' roles morphed and grew beyond the capacity of an academic department (Hoffa, 2007). Many administrators would come to be housed in student affairs or in an academic affairs unit under a midline administrator, provost, or dean. The restructuring for efficiency would ultimately move them away from their access to the core process of the institution and, in some cases, the expertise to assess and evaluate their practice. It is more common now for GDL practice to be housed in a support staff role under an academic or student affairs unit, thus creating a challenge to reach assessment and methodology experts in the technosphere and increasing the challenge of understanding how their practices work and for whom. Without understanding the program theory behind their practices, it is also difficult for practitioners to advocate for their efforts to be a central part of the operating core, despite demand from forces external to the university.

This access to the operating core is a critical element for diversity learning programs, in particular, though it is relevant to global learning as well. Global learning practice administrators often have privileged standing at their institutions, even if funding is scarce, due to their history. Diversity learning practices which tend to focus on differences within local communities began at the margins of the institution and continue to operate there. Ibarra (n.d.) points out how faculty express value for contextual learning like community service, but they do not require it as part of their coursework. This points to a sense from the faculty core that GDL practices are valuable but are not viewed as essential to the process of the organization. While a few GDL initiatives like 
internationalization of the curriculum involve the core process of the institution, most GDLs are considered ancillary to the primary function of the institution. Faculty consider GDL practices as something that is extra, and it shows in enrollment (Olson et al., 2007) Low participation rates in many types GDL programs would also suggest that students do not see GDL as a core component of their process (Kuh, 2008).

Because of their institutional history and socio-cultural context, global learning experiences have enjoyed implicit value and support through federal and private funding agencies (Cornwell \& Stoddard, 1999). They tend to carry influential power with promise of revenue from international students, international travel for faculty and students, and support from industry for the outcomes associated with global experiences. Hovland (2014) points out because of their assumed value the existence of global learning practices and increasing enrollments in higher education witnessed in the 1990s (Altbach, Gumport, \& Berdahl, 2011) have been used as evidence instead of student learning outcomes despite the scant empirical evidence. As global learning administrators look for greater legitimacy in the faculty, they begin to see a path for coalition building through the alignment of outcome goals between diversity and global learning initiatives (Bennett \& Bennett, 1994). Administrative staff connected to diversity learning programs are concerned that working with privileged global learning programs will diminish their ability to meet the goals they see as unique to domestic diversity initiatives.

This preferential treatment of global learning practices in times of austerity has also inspired skepticism from a fairly autonomous faculty body who are less driven by economic power and more motivated by initiatives they see as aligned with their own 
values and mission (Bolden et al., 2012). Faculty, particularly in the sciences, disregard leadership pushes toward GDL practices by assuming that their discipline already represents global and diverse perspectives because scholarship in their field comes from many countries, a view which overlooks language dominance and domestic diversity (Agnew, 2012). Faculty who align with diversity learning coalitions question the motives of university leadership as they use international students to increase tuition revenues, appease industry stakeholders, and meet student diversity targets (Olson et al., 2007).

Without access to technical research and evaluation skills to demonstrate GDL practice outcomes for a wide range of students, leaders in GDL practices must use financial incentives, loss of accreditation threats, or other soft power coercive tools to advance toward their goals. Faculty are unlikely to view GDL practices as critically important to the institution or their own practice unless they are already personally aligned with GDL outcome values, creating a challenge for university leadership.

\section{Leadership Analysis}

The prior section provided a description of political and structural context for GDL practices at many US universities. This section will provide background on university leadership dynamics within that context. The purpose of this discussion is to demonstrate how senior leadership responsible for GDL practices (e.g., senior international officers, diversity officers, and student affairs directors) could benefit from enhanced empirical knowledge of the outcomes of different types of GDL practices. Through authentic leadership and well-designed research on the outcomes of GDL practices, senior GDL practice leaders will be able to more effectively navigate troubled 
relationships between university top leadership and faculty members and find their way into the core function of the institution.

Top university leaders sit in a unique place, across the table from leaders in industry, civic organizations, and government who hope to have influence over which skills and dispositions graduates acquire. Nevertheless, leaders at the top of a university are not often vested with the same types of positional power that leaders of businesses and other organizations hold (Birnbaum, 2004). Where conventional business owners may have the power make strategic decisions in isolation if they choose, this is not typical of a university president. Their titular role does relate the expectation for shared governance held by faculty. Increasingly, university leaders in the US are spanning funding gaps in ways that faculty perceive as undermining their legitimacy and right to shared governance (Birnbaum, 2004). Birnbaum further cautions that shared governance models where faculty are given power to control the core process of the institution while administrative leaders control the structures that support the core function are being tested in a growing era of neoliberalism. Voices from industry stakeholders are often at odds with faculty members who view their role as one that develops an educated citizenry capable of transferring knowledge and adapting to changing economic circumstances. While industry leaders are more inclined to promote a model where faculty are purveyors of career-related information, students are customers who want to obtain a degree as quickly and uneventfully as possible (Birnbaum, 2004). Del Favero and Bray (2005) point out this classic tension in shared governance between leadership and faculty. They suggest that university leaders are acting with the collective, resource-scarce view in mind while faculty are driven to decisions based upon individual area interests and 
personal values. Industry voices express the need for employees who can help them gain advantage in new markets through understanding how to successfully interact across differences (British Council, 2013). Faculty members tend to point to how this frame is exploitative and perpetuates inequities in society (Gorski, 2008). Senge and Kofmann (1995) suggest these increasingly polarized values can fragment organizations.

In this context, top university leaders tap senior international officers who are traditionally outside of the faculty corps to facilitate new learning outcomes. This creates a difficult environment for senior international officers to navigate. Without modifications to the core process of the university, how can institutions produce graduates who are able to successfully and sustainably work in an increasingly diverse workplace? Yet, international and diversity officers have traditionally not been trained in learning assessment and have no direct access to the entire study body. Additionally, these GDL leaders must rely on faculty to feed students into their practices, so being seen as a way to circumvent faculty governance and input on learning outcomes could make GDL leaders' jobs more difficult. In the end, many GDL leaders rely upon student participation numbers or surveys of student satisfaction to act as a placeholder for learning outcomes (Lou et al., 2012; Whitehead, 2015).

Which type of leader can make meaning of the competing demands and take action to change their institution to promote equity, democratic values, and cohesion in their communities? Leadership scholars (Brewster, Dyjak-LeBlanc, Grande, White, \& Shullman, 2016; George, Sims, McClean, \& Mayer, 2011; Goleman, 2011) suggest the same competencies demanded of university graduates are competencies leaders must develop in order to be effective in an increasingly entangled and complex society. Lack 
of explicit awareness of one's values, beliefs, and motivations leave leaders subject to follow the example set by predecessors, whether it was productive or not. They maintain the status quo through feedback mechanisms benchmarked on current practice rather than values as Birnbaum (1989) describes.

Carrying out GDL programs that are not well understood through offices which faculty see as ancillary support to the core function of the institution sends a signal to faculty whether leadership intends to do so or not. In the current tug-of-war, university leaders point to the industry demands for twenty-first century skills and faculty push back in two ways: to reject the premise that industry could be a legitimate stakeholder for setting learning outcomes and to point out that they are not trained for or rewarded through tenure and promotion processes for the development of awareness of self and other in their students (Birnbaum, 2004; Kahn \& Agnew, 2017).

University leaders do not currently have access to sufficient evidence to make claims to internal stakeholders regarding which GDL practices produce learning outcomes they target at their institution (Altbach et al., 2011; Kuh. et al., 2015; Lou et al., 2012; Ogden, 2010, 2015). To produce evidence of learning in GDL practices, university leaders are turning to senior international officers who often are positioned outside the faculty body. Marginalization of these practices then privileges small groups of students and leads to increases in the achievement gap (Kuh, 2008). Production of evidence of learning has traditionally been a role played by faculty members who share university governance with leaders at the apex of the organization.

How can senior international officers promote cohesion across campus through further investigation of GDL practice outcomes? The baggage that comes with history of 
these types of programs will need to be addressed by leadership. Through authentic leadership, progress to better understand the context in which these outcomes develop for different types of students can be made. Senior international officers could be in a place to lead through shifting institutional paradigms, demonstrating transparency and authenticity in their pursuit to forge relationships with the faculty body that would allow integration of GDL practices into the core function of institutions.

Kahn and Angew (2017) suggest that GDL leaders and faculty first need to shift their perspectives on knowledge production and translation. They posit that US institutions are stuck in a positivist, bureaucratic paradigm in most institutions of higher education. Kezar et al. (2006) suggest that it is necessary to move away from positivist understandings of leadership in higher education. It is the role of the leader, scholarpractitioner to understand organizational culture in order to push against conventional modes of creating knowledge, pigeon holing global learning into a discussion of culture. Woolf (2013) also warns against the comfort of consensus that could come with the professionalization of a leadership role. He points out how selection processes for GDL leadership positions, in international education in particular, select against those who have not participated in the practice, thereby creating a unit that does not easily understand the non-participant experience and perpetuating the low participation rate.

Someone from the apex of the organization and the core process must ultimately be involved in promoting and assessing GDL practices. Without the buy in and support of leadership, however, it would be difficult to gain the attention of the most important unit of the institution, the faculty. How to approach faculty, particularly on campuses where diversity and global initiatives are housed separately, is a challenge because many 
faculty see these initiatives as unessential aspects of the function of the institution. Many faculty also suggest that they have no training, and therefore no role, in facilitating awareness of self and others and to a lesser degree critical thinking (Olson et al., 2007) Goode (2008) documented the lack of professional development in these areas for faculty. He indicated that faculty do not feel they have enough training or do not feel they have a role in the development of self and other awareness. Most of the faculty in the study were uncommitted to the importance of developing these skills during a study abroad program. (Goode, 2008).

In this context, it is important for GDL practice leaders to understand how different types of programs influence student capacities. Partnering with faculty who have the methodology training to support GDL practice evaluation and assessment is a potential step toward integration of GDL practices to the wider student body. Without some initial empirical evidence, however, it may be difficult to get faculty members' attention in the first place.

\section{Implications for Research in the Practitioner Setting}

The structural and political challenges that leaders in US higher education face to promote and expand GDL practices could be met through promotion of paradigm shifts in how the university demonstrates and talks about student learning outcomes. Authenticity and transparency are key qualities that leadership in higher education will need to employ to understand why and to what end they promote GDL practices.

This research study with its practitioner product could help provide leaders in GDL practices the evidence they need to meet with faculty and discuss alignment of 
goals and integration into the core process of the university, as faculty will be reluctant to make changes to the curriculum or degree-granting process without sufficient evidence.

\section{Summary}

The divergent history of global and diversity learning practices has led to a slow start in collaboration between university support staff charged with facilitating activities that scholars believe could have similar learning outcomes. Leaders who have privileged global learning over diversity learning have lost the trust of faculty and diversity learning staff. This mistrust and isolation within the institution creates inefficiencies and conflict between units. The isolation also contributes to a privileging of certain groups of students over others when it comes to who can participate in GDL practices. In order for university leadership to bring faculty together with both global and diversity learning practitioners, efforts to be transparent and authentic are needed to inspire trust and hope. 


\section{SECTION THREE}

LITERATURE REVIEW 


\section{Literature Review}

High Impact Practices (HIPs) became a commonly used term in US higher education in the late 2000s. This set of ten categories of disparate educational practices that are intended to produce deep learning has inspired considerable discussion in the scholarly community. Kuh's (2008) foundational report codified how future scholars would categorize and view experiences as varied as undergraduate research and studying abroad (Kuh \& O’Donnell, 2013). That report established vocabulary that would be used across the US in higher education circles to discuss how universities would produce and demonstrate the production of students with key twenty-first century skills.

This literature review begins with a concise overview of the vocabulary and rationales behind HIPs. The review then narrows to a specific category of HIP, global and diversity learning practices (GDL), involved in this study. A discussion of how to bring such a wide array of practices, even within one HIP category, concludes the review of literature.

\section{Theoretical Framework}

Something notably missing from discussions about HIPs is a theoretical framework that can encompass such different experiences aimed at common outcomes. Prior to Kuh's (2008) work, the experiences that would come to coalesce into ten HIP categories, lived in different worlds on US university campuses. Research on those practices was primarily done without consideration of other types of practices that might produce similar outcomes. Additionally, each scholars brought a different set of theoretical frameworks to explain why he or she should expect the outcomes being investigated. For example, scholars in international education in the US, have 
historically used Milton Bennet's (1998) Developmental Model of Intercultural Sensitivity as a framework for the experiences of leaving one cultural community to join another (Bolen, 2007; Engle \& Engle, 2004; Twombly et al., 2012). Scholars concerned with diversity education tend to use Critical Theory as a framework for the experiences of minority students on US campuses. Without identifying a common framework, it is challenging to establish valid program logic and evaluation methods.

One common element about the HIPs is the emphasis on connectedness and relationship among all participants in the learning environment. The following section outlines how two frameworks, one for understanding the internal psychological processes within the student and another for understanding the pedagogical aspects of the learning experience, could be useful in establishing a sound logic model for GDL practices.

In considering processes of developing awareness of self and other and critical thinking, it is necessary to look for frameworks that provide understanding of how the self is created and maintained as well as which elements of the self are changed when learning occurs. There are two complementary theoretical frameworks that are useful in explaining why one can expect GDL programs to bring about changes in world view, increasing self and other awareness as well as complex thinking skills. In the section that follows, a discussion of Transformative Learning Theory (Dirkx, 1998; Hoggan, 2016; Mezirow, 1997) and the EI Theory (Shealy, 2004, 2006, 2015) frames this study. EI Theory provides the framework for understanding how students develop the worldviews that they bring to interventions, while the Transformative Learning Theory provides a pedagogical structure for understanding how that worldview can be changed through an intentionally designed learning experience. 
Equilintegration Theory seeks to explain "the processes by which beliefs, values, and worldviews are acquired and maintained, why their alteration is typically resisted, and how and under what circumstances their modification occurs" (Shealy, 2004, p. 1075). An element of EI Theory is that while beliefs and values moderate behavior at individual and group levels, those beliefs and values may not be known explicitly or rational (Shealy, 2006). EI Theory outlines a developmental model that begins with infants acquiring understanding of the world through the context that surrounds them (Shealy, 2004). Individuals develop layers of complexity as they begin to think of themselves as individuals and have internal dialogues, taking stimulus from the external environment, and making meaning from it. Shealy (2015) explained that ...belief and value content (i.e., the beliefs and values that a human being holds to be self-evident about self, others and the world at large) largely is a function of those beliefs and values that predominate in the primary cultures and context in which that human being develops and lives (p. 96).

Through this framework, we begin to understand how students come to learning experiences with a particular worldview. Furthermore, we can see how a simple lack of awareness, through living in a mono-cultural community or through psychological need, of that worldview may prevent students from changing their perspective or attitudes.

According to EI Theory, individuals are composed of beliefs and values that are available to them as they develop (Shealy, 2004; Vygotsky, 1997). Through novel experiences that are not consonant with their prior experiences, individuals may begin to be aware of their beliefs and values. Therefore, if true change in awareness or critical 
thinking is to occur, some sort of intervention may be necessary to act as a catalyst (Dabrowski, 1964; Dirkx, 1998; Dweck, 2008; Mezirow, 1997).

While EI Theory is useful in bringing us to an understanding of how students may enter and need the intervention, it does not entirely offer a framework to bridge to pedagogy necessary to elicit change - the catalyst. It is necessary, therefore, to employ Transformational Learning Theory (Mezirow, 1997) as a framework for which changes in the student would bring about greater awareness of self and others as well as critical thinking. For the framework of this study, it is through transformational learning experiences that students become aware of their worldview.

Transformative Learning Theory is, "the process of effecting change in a frame of reference" (Mezirow, 1997, p. 5). In his theory, Mezirow outlined ten steps involved in the process of affecting change that have been critiqued and modified over the past two decades. Hoggan (2016) completed a meta-analysis of studies in transformative learning and developed a taxonomy to describe essential components of transformation which were present in the majority of studies he reviewed. His work outlines five fundamental components: (a) a change in worldview, (b) changes in ways of knowing, (c) changes in cognitive ability, (d) increased self-awareness, (e) increased mindful awareness of subconscious dialogue, and (e) changes in action.

Combining EI Theory and Transformative Learning Theory, provides a context for understanding where students acquire the awareness and critical thinking with which they enter a given GDL practice, why their core needs might prevent them from wanting to change those capacities, and which components of the self must change in order for 
transformative learning to occur. The specific transformative learning interventions involved in this study are described further in the following section.

\section{High Impact Practice: Rationale and Definitions}

In the past twenty years there has been a growing demand for universities to demonstrate targeted student learning outcomes (Brownell \& Swaner, 2010; Ogden, 2015; Sutton \& Rubin, 2004). Employers have complained that collegiate learning is not producing culturally competent graduates with critical thinking and complex reasoning skills (Green, 2013). As employers expand their markets, they claim their workforces must be able to interact successfully with people whom they perceive as different and understand geopolitical realities (Friedman et al., 2015).

The ability to think critically in a novel environment and interact with people who are culturally different are outcome goals that both corporate and higher education leaders across the globe are targeting (British Council, 2013; Hart Research Associates, 2008). As demand for these particular types of skills increased, American Association of Colleges and Universities (AAC\&U) launched the Liberal Education and America's Promise (LEAP) campaign in 2005. The campaign sought to develop 21st century skills in university students - particularly those who have not traditionally had access to college (Brownell \& Swaner, 2010). LEAP was intended to help universities keep up with the pace of changes in the workforce by organizing national discussions about the current needs of employers, establishing a research agenda to provide evidence of the outcomes associated with a liberal arts education, and setting up a public advocacy campaign to promote liberal education to business leaders and policy makers (Brownell \& Swaner, 2010). 
The LEAP initiative would later develop into Kuh's (2008) work. In looking through university learning practices, Kuh coalesced ten categories of learning experiences that showed potential for the types of outcomes administrators and the business community sought. The ten categories are (a) first year seminars, (b) common intellectual experiences, (c) learning communities, (d) writing intensive courses, (e) collaborative assignments, (f) undergraduate research, (g) service and community-based learning; internships, (h) capstone projects, and (i) global and diversity learning. In the introduction to Kuh's description of these HIPs, Schneider (2009) suggests the common learning outcomes of these practices should be: (a) understanding that there are many human cultures in the world, (b) developing intellectual as well as applied skills, (c) recognition that one has responsibility for self and others, and (d) the ability to apply learning (Kuh, 2008; Maki \& Schneider, 2015).

With the practices that could facilitate these learning outcomes identified, The Association of American Colleges and Universities (AAC\&U) established commissions for further reports that would compile data on what research had been done on each of the categories. Kuh (2008) suggested that these experiences had an added bonus of increasing student performance overall—potential to close achievement gaps. Unfortunately, the types of practices recommended by these studies were traditionally used by non-minority students (Maki \& Schneider, 2015). Students engaged in high impact practices still tend to be Caucasian and middle to upper class (Kuh \& O’Donnell, 2013).

In addition to being activities used by a select group of predominantly White students, HIPs came under more criticism because there was scant and disjointed 
empirical evidence for achievement of learning outcomes (Brownell \& Swaner, 2010). In subsequent publications, Kuh and O'Donnell (2013) caution that not enough data has been collected to truly warrant the label 'high impact' in all cases.

Implicit value for HIPs like education abroad and the complexity of targeting learning in such deep experiences led universities to increase funding for HIPs without the empirical evidence for the individual learning outcomes targeted through the LEAP initiative (Lou et al., 2012). The internal outcomes for individual students are less clear because the variables increase exponentially when one considers who a student is prior to the experience. Additionally, isolating the components of the various programs that could account for the change that occurs in students is complicated by the wide array of practice types and configurations. High impact learning initiatives like global learning and diversity initiatives tend to be complex, multi-component experiences whose individual contribution to changes in student psychological outcomes like self- awareness and other-awareness are difficult to isolate. In the current, polarizing climate in the US, with many campuses grappling with how to create cohesion amidst rising tension over race and gender identity, it is imperative that the academic community explore how these high impact initiatives change students' ways of thinking and perceiving the world to become more open to hear about the lived experiences of 'the other.'

\section{Global and Diversity Learning Programs}

Nested under the umbrella of HIPs, global and diversity learning programs (GDLs) are made up of experiences like education abroad, global or multicultural livinglearning communities, global studies courses, and multicultural programming (Whitehead, 2015). The push for global learning is, to some, an indication that 
universities are shifting from a focus on instruction in a singular classroom context to one of learning across an array of authentic environments (Green, 2013; Kahn \& Agnew, 2017). GDL programs expand past the traditional study abroad boundary. They support students as they encounter differences that will make them uncomfortable or question their worldview. These types of programs should have content that encourages students to think about social justice struggles (Sandeen, 2012).

While the push toward GDL programs is widespread, agreement on definitions of global and diversity learning is not (Hovland, 2014). The American Council on Education's efforts to promote a shared definition have resulted in the development of a global learning VALUE rubric to assist universities as they seek to align learning outcomes with institutional visions (Hovland, 2014; Kahn \& Agnew, 2017; Whitehead, 2015). The word global was intentionally used to include an emphasis on sociocultural contexts that are not constrained by political boundaries. It also allows for learning outcomes that focus on the entangled interactions of multi-layered local contexts (Hovland, 2014).

Outcomes commonly associated with GDL programs range from increases in awareness of self and others (DeTurk, 2006; Lou et al., 2012; Ogden, 2006; Wandschneider, Pysarchik, Sternberger, \& Ma, 2015) to increases in critical, complex, and creative thinking (Galinsky \& Maddux, 2009; Grunzweig \& Reinhart, 2002; Hammer, 2009; Law, 2014; Lou et al., 2012; Stebleton et al., 2013; Wandschneider et al., 2015). Despite some associations, there is still agreement that the connections between these program types and outcomes are tenuous (Kuh. et al., 2015; Lou et al., 2012; Wandschneider et al., 2015). Additionally, the body of literature that would help the field 
understand the linkage between what the students bring to experiences and the specific types of GDL practices is quite small (Salisbury, 2015; Wandschneider et al., 2015). Understanding the interactions between the types and outcomes of GDL practices is critical in order to be able to create sustainable programs that produce predictable outcomes.

GDL program components. Efforts to identify and operationalize component elements have predominantly erupted in education abroad literature, though calls to develop a framework for a broad range of GDLs exist (Engle \& Engle, 2003; Salisbury, 2015; Wandschneider et al., 2015). The section that follows will describe the program components that have been proposed in the literature that are also part of this study. A discussion of the gaps that exist and this study's contribution will conclude the literature review.

Duration. This GDL program component is one of the more examined elements among all types of GDL programs. Among the wide variety in GDL programs, there is no common unit of measurement, e.g., hours, weeks, or months. Additionally, intensity, a facet of duration, is often overlooked (Wandschneider et al., 2015).

In the area of education abroad, scholars have sought to understand how duration impacts a variety of learning outcomes. Engle and Engle (2004) were surprised to find that increasing program duration in French immersion programs did not produce proportional yields in language proficiency but did produce the expected proportional gains on intercultural awareness. The Georgetown Consortium Project (Connor-Linton et al., 2009) found similar results in regard to intercultural awareness, however, in both studies, sample sizes limited the findings. Additionally, neither study considered the 
intensity of the program, i.e., how many hours students were expected to be on task with program objectives.

Depth of experience. Immersion, contact hours, and engagement are just a few terms that scholars have used to describe depth of experience (Engle \& Engle, 2004; Iyer, 2013; Lou et al., 2012). Again, due to the wide array of GDL program types, scholars do not use the same language or constructs in their research from one program type to another. The notion that some amount of time must be spent in meaningful contact with others for the purpose of learning is something many researchers assume in their studies. Wandschneider et al (2015) describes depth as, "the capacity of the learner to experience all that the intervention is able to convey" (p. 217). Salisbury (2015) focuses more on the design of the experience. He suggests that depth is the "degree to which students interact" (p.42) and "the nature of those interactions" (p. 42).

Program design for transformative learning. This component is as varied as the others in this list in its representation in the literature. In essence, this component represents the intent and alignment of the learning outcomes and learning activities. Salisbury (2015) uses the term "pedagogical approach" while Engle and Engle (2003) focus on the collection of activities that are intended to bring about change in students.

Engaging difference. This component differs from the others because, unlike the other components, scholars tend to use the same term. However, their notions of difference and the outcomes associated with encountering it are quite different. For some engaging difference is situated within the participant, how different is the learning context from what the participant perceives as familiar (Iyer, 2013; Preston \& Peck, 2016; Shealy, 2015; Wandschneider et al., 2015). For other scholars and program 
designers, engaging difference is less abstract and focuses on geopolitical, racial, and other easily quantifiable constructs of culture, values, or behaviors (Connor-Linton et al., 2009; P. Gurin, Dey, Hurtado, \& Gurin, 2002b; Hathaway, Pilonieta, \& Medina, 2015; Hudzik, 2011; Richart, 2015). The underlying assumptions of the outcomes of encountering difference are also somewhat varied. Some scholars base their assumptions on Transformative Learning Theory (Mezirow, 1997) predicting that encounters with difference will be inspiration for the disequilibrium moment necessary for change (Iyer, 2013; Ogden, 2007; Wandschneider et al., 2015). Other scholars seem to base their assumptions about encountering difference on Contact Theory (Allport, 1954) where encountering difference in the right context promotes understanding (Bennett, 1998) and decreases stereotyping behaviors (Hathaway et al., 2015; Law, 2014; Lou et al., 2012).

\section{Outcomes Related to GDL Practice Types}

The types of outcomes that have conventionally been captured from GDL practices are similar to those from the wider array of HIPs. Several scholars have noted that the traditional approaches to assessing these types of practices that include analysis of participation rates, GPA changes, or persistence to graduation rates are no longer adequate to warrant the label of 'high impact' (Brownell \& Swaner, 2010; Hovland, 2014; Kuh \& O’Donnell, 2013). Moving away from participation outcomes, employer's and democracy advocates' interests in 21 st century skills and global competencies have put pressure on universities to demonstrate that they are producing students with capacities like awareness of self and others and critical thinking (Connell, 2016; Hart Research Associates, 2016; Sandeen, 2012; Whitehead, 2015; Wilson-Mulnix, 2012). 
Shifting away from variables that are easily accessed, like GPA or participation rates, has posed a problem. As Whitehead (2016) points out, there is no single measure for capturing outcomes of GDL practices. Therefore, it becomes challenging to compare results of studies because each measure conceptualizes outcomes in a slightly different manner. For example, Conner-Linton, Paige and VandeBerg (2009) studied education abroad programs and focused on intercultural awareness outcomes. Within intercultural awareness, there are components of awareness of self and others. Deardorff (2009) points out how these facets of intercultural awareness are essential for one to possess in order to have successful interactions with people from diverse backgrounds. However, because the constructs, instruments and frameworks used in various studies of GDL practices differ, it is a challenge to corroborate findings between studies. Additionally, there are multiple approaches to measurement of awareness of self and others, and critical thinking. Several studies that have analyzed outcomes of GDL programming focused on indirect measures of awareness of self and others and critical thinking (Braskamp, Braskamp, \& Merrill, 2009; Kilgo, 2016; Kuh \& O’Donnell, 2013). These studies relied upon student perceptions of their awareness and critical-thinking capacities. From an EI Theory perspective, it is not ideal to rely upon the self to assess capacities that it cannot explicitly or consistently conceptualize or identify (Shealy, 2015). GDL practice studies that use direct measures of changes to the self (Connor-Linton et al., 2009; Iyer, 2013; Kilgo et al., 2015; Wandschneider et al., 2015) do not always use the same language for constructs that involve awareness of self and others, as in the example above regarding intercultural awareness. However, because there is not a large number of studies on GDL practices of any type focused on awareness of self and others, or critical thinking; it is 
necessary to encompass many approaches and related conceptualizations of the three outcomes involved in this study. In the section which follows, there is a discussion of prior GDL practice research that addresses one or more outcomes associated with this study. It is important to note that the author could not find any publications that considered the contribution of GDL practice type to the outcomes of awareness of self and others and critical thinking. Therefore, the section below is organized by outcome and contains studies that considered single practice types.

Self-awareness and other-awareness. These constructs are presented together here because of their entangled nature and the prevalence of studies that involve the combination of these two constructs enveloped in a larger construct such as intercultural competence.

Education abroad. In the past decade, a growing number of studies of GDL practices have targeted intercultural awareness, also known as intercultural competence. As noted in Section 1, the shift from area studies and content knowledge in the post-Cold War era, coupled with the public demand for universities to demonstrate learning, pushed international educators to identify desirable learning outcomes that could be developed through education abroad experiences (Bolen, 2007; Hoffa \& DePaul, 2010; Lou et al., 2012; Ogden, 2015). One of the higher profile studies involving education abroad and its impact on intercultural learning outcomes in students is the Georgetown Consortium Report (Connor-Linton et al., 2009). This study involved 1,297 participants, 138 of whom were a control group participating in domestic intercultural coursework, from 190 universities in the US. Researchers found significant differences in intercultural competence scores between the study abroad students and control group students, with 
greater pre-post-experience gains in scores for study abroad students. However, these gains were specific to females in the study abroad group, a finding supported by findings of Braskamp and Engberg (2011). Georgetown researchers (Connor-Linton et al., 2009) found no significant gains in intercultural competence in male study abroad participants. Braskamp and Engberg (2011) found that women scored higher than men on measures of awareness of others as it relates to social responsibility and openness to encounter different perspectives.

Intuitively, it makes sense that students might gain more awareness of self and others if they had already participated in a high impact practice. Kuh et al (2015) suggest that learning outcomes are compounded when multiple HIPs are combined. However, studies in education abroad are mixed in this regard. For the Georgetown study (ConnorLinton et al., 2009), prior experience studying or living abroad did not impact awareness of self and others in the form of intercultural score gains. However, those who had been abroad less than one month prior to education abroad participation did realize the greatest gains overall. Interestingly, students who had studied language prior to participation, did see significantly higher gains in intercultural competence than those who did not.

Age was also a factor in the Braskamp and Engberg (2011) study. They found that students over the age of twenty-five had higher scores on self-awareness aspects like identity as well as higher scores on other-awareness aspects like emotional attunement and responsibility for others. Few other studies have included age as an important factor; however, with the increasing number of non-traditional students entering or returning to higher education, it is important to consider this variable as an influential component of student learning outcomes. 
Multicultural programming. As with education abroad, multicultural programming takes many shapes on college campuses. Experiences could include a speaker or film series on diversity or intercultural issues, first-year diversity training, intergroup dialogues, or any other activity intended to increase positive interaction between students from a wide array of experiences and backgrounds. Also, like education abroad, the outcomes of awareness of self and other are operationalized in many ways. A few examples that describe patterns in outcomes are discussed briefly in this section.

Intergroup dialogue is an educational endeavor intended to "bring together students from two different social identity groups in a sustained and facilitated learning environment." (Nagda et al., 2009, p. 2). As Nagda and colleagues point out, the purpose of intergroup dialogue is designed as a pedagogical tool for getting students to address issues around diversity and injustice in society, and reflect on their social responsibility. Research on this type of GDL reveals strong patterns of increased awareness of self and others through dialogue. In a comprehensive analysis of studies on intergroup dialogue published between 1997 and 2006, Dessel and Rogge (2008) identified many studies where participants in intergroup dialogue increased awareness of self and others through perspective taking behaviors, and increased self-confidence for interacting with peers from differing backgrounds, as well as increased awareness of their own values and beliefs. More recently, in a quantitative study of first-year student experiences in intergroup dialogue, Thakral, Vasquez, Bottoms, Matthews, Hudson, and Whitley (2016) found that freshmen gained on measures of openness to difference of perspective and awareness of different lived experiences of peers due to social inequities. 
Living-learning communities. As defined in previous sections, these types of communities can center on a variety of disciplines or topics and may include faculty in residence. The common element among living-learning communities (LLCs) is the element of students sharing living space and studying a common theme over some span of time. Studies around LLCs have identified a wide array of outcomes such as increased retention (Lenning \& Ebbers, 1999; Purdie \& Rosser, 2007; Tinto, 2006); increased capacity to see multiple perspectives (Brower \& Inkelas, 2010; Inkelas \& Weisman, 2003); and increased awareness (Iyer, 2013). Few studies, however, focus on LLCs that have a focus on multicultural or intercultural content. The discussion that follows highlights one large-scale study of LLCs in general and one case study of an LLC focused on global learning.

In 2011, Kuh, McCormick, and Pike reported on a multiple regression analysis they completed using a large-scale dataset called the National Survey of Student Engagement, or NSSE, (Indiana University Center for Postsecondary Research, 2007). This survey was used across the US to collect student perceptions of the impact of their engagement in college life. Kuh, McCorkmick, and Pike (2011) found that students who participated in learning communities, in general, were more likely than those who did not to encounter peers from different backgrounds. Similar findings also appear in Braskamp and Engberg's (2011) study of roughly 5,000 students from multiple institutions in the US. Being female or a senior and participating in an LLC demonstrated even greater increases in contact with diversity.

An in depth case study by Iyer (2013) illuminated the outcomes of student interactions with diversity. In her work, Iyer found that a diverse group of students living 
together and learning about how they develop their identities and worldview were able to report increases in self-awareness through identity clarification, capacity for introspection, open-mindedness toward beliefs, and acquiring view of self as a change agent. Additionally, she found that students perceived they were able to increase their capacities to identify beliefs and values in others, respect differences in worldviews, and increase their feelings of social responsibility and empathy for others.

Global studies courses. Research on this type of GDL practice is different than the other categories because global studies courses are typically part of a degree program or a course used in fulfilment of general education requirements. In the literature, global studies courses are typically used as an alternative or comparison group for education abroad outcomes. The rationale for the comparison is that students who choose not to study abroad but participate in global studies have similar interests in learning about the world. Whether this is founded or not, comparison studies are the most common format for research on global studies.

Soria and Troisi (2014) used a large dataset of student engagement responses from the Student Engagement in the Research University survey (University of California-Berkeley, 2014) to do this type of comparison between global studies, education abroad, and domestically based intercultural experiences. They used intercultural competence as an umbrella construct for awareness of self and others. Through use of multiple linear regression analyses, they found that some demographic variables were consistently related to students' self-reported development of GII [Global, Intercultural, International] competencies. For example, females and first-generation, low-income, and working-class students 
self-reported greater development in both GII competencies. Furthermore, age and academic credits earned were consistently positively associated with students' self-reported development of both GII competencies. (Soria \& Troisi, 2014, p. 271)

They also found that while participants in both global studies courses and education abroad experiences showed increased openness to difference after participation, mean scores for the global studies group were higher. This does not account for the potential that those opting into the dialogue group started with higher means, however.

Critical thinking. As with awareness of self and others, critical thinking has no commonly approached method of operationalization. Therefore, many of the studies exploring the relationship between GDL practices and critical thinking have varied results. Notable studies are discussed in the following section with comments regarding patterns that emerged.

Education abroad. Content instruction in area studies was the focus of education abroad programming for decades after World War II (Hoffa, 2007). Learning outcomes connected to critical thinking were then tied closely to analysis and comparison of historical, economic, and political systems. It has only been within the past decade that the construct of critical thinking, broadly constructed, has been an outcome of consideration in the field of education abroad. As with awareness of self and others, results are somewhat mixed as are the methods of operationalization of the construct itself.

Braskamp and Engberg (2009) identified a nuance between the cultural content knowledge acquisition and critical thinking in their study of over 5,000 students. Those 
who had spent a semester abroad demonstrated higher cultural content knowledge than students who did not study abroad, but the study abroad participants did not demonstrate critical thinking capacities that would allow them to apply that knowledge in culturally appropriate ways. These findings are somewhat at odds with a smaller comparative study of students who signaled interest in study abroad, had studied abroad, or had no intention of studying abroad (Lee, Therriault, \& Linderholm, 2012). Through use of an ANCOVA to compare the impact of group membership to outcomes on critical and creative thinking measures, the research team found that "cultural experiences from living abroad have wide reaching benefits on students' creativity including the facilitation of complex cognitive processes that promote creative thinking in multiple settings" (p. 775). The effect for female participants was significantly larger than male participants. In a different study comparing study abroad to a range of other high impact but non-GDL practices, Kilgo et al. (2015) found that study abroad did not have a significant impact on critical thinking gain scores in comparison to other HIPs. The inconsistency of findings could be attributed to a number of factors from differences in program design to how the constructs were operationalized.

Multicultural programming. Important outcomes connected to critical thinking that appear in the literature are how students connect the knowledge they receive from diverse perspectives and shared stories to the larger systems that are in place that create inequities and injustice. Thakral and colleagues (2016), in their analysis of the outcomes of an intergroup dialogue program on race, found that students in the dialogue group were able to think more complexly about the systemic patterns of racial inequality compared to the control group. 
Living-learning communities. Multiple studies found connections between participation in LLCs and changes in some form of critical thinking (Inkelas \& Weisman, 2003; Iyer, 2013; Pike, 2008; Pike et al., 2011). The in-depth study of a global learning LLC by Iyer (2013), however, is unique in how it connects students' emotional capacities to the critical thinking gain scores. She notes that some students had a way of protecting their internal values and beliefs through avoiding cognitive awareness of emotions. Iyer notes,

This response seems to be caused by a fixed view about self, others, and the world, which therefore struggles to grasp the complexities of human behavior. Such an experience may be due to fixed affective/cognitive structures characterized by strongly held beliefs about "right and wrong," which may be associated with black and white thinking, and the conflict between course material and one's own beliefs and values. At the same time, there may be a deep desire to come to terms with affective responses, and to make sense of them in a way that allows one to move forward in a more integrated manner. (p. 89)

Braskamp and Engberg (2011) made similar but less sophisticated observations of the connection between critical thinking and affect, however, neither study used methodology that would allow for directionality in the associations. It is possible that students who have higher emotional capacity are drawn to experiences where they will encounter new ideas and diverse worldviews.

\section{Conclusion}

It is worth noting the entangled nature of program components in general. Changes in one facet of a program could potentially have impact on another. For 
example, reducing the duration and intensity of an experience would arguably impact the depth participants are able to reach in their interactions. Wandschneider et al. (2015) stress the importance of exploring these relationships more completely. There are a number of studies that investigate interactions between two variables in isolation like program duration and awareness gain scores (Dwyer, 2004; Medina-Lopez-Portillo, 2004; Sutton \& Rubin, 2004). It is important to acknowledge the entangled and complex nature of experiences that are designed to fundamentally change internal processes. Focusing on time and its relationship to intercultural awareness outcomes leads to conclusions that overlook the interaction of other variables. Because there are no reasonable ways to randomize participation in GDL programs, it is not possible to ascribe causality for the types of studies done in this sector. Accounting for a wider array of variables is, therefore, even more critical to understanding potential impacts of the program as a whole.

With support for reflection in a well-designed experience, students can reorganize their perspectives to see the world more complexly and act more effectively in that world (Shealy, 2015). Understanding the relationships between program types and student characteristics and outcomes is the first step toward providing leaders in US higher education data points they need to make informed choices about resource allocation. The current body of knowledge on GDL programs, however, is inadequate for administrators to make those decisions (Kuh et al., 2015; Kuh \& O’Donnell, 2013; Salisbury, 2015; Wandschneider et al., 2015). 


\section{SECTION FOUR \\ CONTRIBUTION TO PRACTICE}

Reader's Note: The slide presentation and handouts included in this section were developed for a pre-conference research roundtable session at the NAFSA 2019

international conference. This is the conference where international education practitioners come for professional development and networking. The pre-conference workshops and roundtables target practitioners who want to learn deeply about specific topics. During the first part of this session, I will present the findings of the study and ask participants to sit at tables with peers from institutions with similar outcome goals. During the second part of this session, participants will work through the program theory/assumptions of learning with their group, using the logic model I will provide. The goal of this part of the session will be to have participants identify student characteristics from the study that serve as inputs to their international education programs. Based upon those inputs, I will ask small groups of participants to identify how those student characteristics (e.g., gender) may interact with the activities in their programs to impact learning outcomes. 


\section{Application of Findings for Practitioners}

One of the most uncomfortable moments in my professional life occurred while I was a study abroad advisor at a public ivy university. My director pulled me into a meeting with six faculty members who composed the Education Abroad Advisory committee. It was a newly founded committee of faculty members who had experience taking students overseas. The task my director wanted me to accomplish was to establish a system for evaluating faculty-led programs to determine whether they were meeting learning objectives that our office had recently established to align with the overarching university mission. What I needed in that moment, aside from a shred of positional power, was empirical evidence and a framework for the types of outcomes that were realistic to expect from different types of programs. At my institution faculty led students on a wide array of different program experiences, and they were reluctant to accept a set of standards that included outcomes they saw as outside of their disciplines. Results from a study like this one would have allowed a conversation about which types of outcomes are realistic to expect from education abroad experiences and other types of global diversity learning (GDL) practices taking place on campus. The results of this study would also have inspired a conversation about know who students are prior to their participation in GDL practices.

Additionally, outcomes of this study would have allowed me to identify other GDL practice offices with similar outcomes to whom I could reach out for collaboration and support. When I reflect back to my role as an education abroad adviser, however, I realize that I lacked the information and a context for bridging the conversation with my peers regarding how and why we might collaborate. 
The results of this research demonstrate that there are multiple GDL practice types that produce the same learning outcomes, but with differential effect. In our roundtable discussion group for practitioners at the NAFSA 2019 conference we will discuss the results of this study and project the findings onto our practices. The summary and handouts below will give you the background that you need to prepare for the round table session.

\section{Executive Summary of the Study}

The ability to think critically and complexly amid novel experiences that require awareness of self and others is something that leaders in an array of social institutions seek to develop in their communities. Global and diversity learning practices, one category of high impact practices (Kuh, 2008), aim to increase students' awareness of self and others and imbue critical thinking skills that will help students see how their own background and experiences interact with those of peers. This study aimed to understand the relationship of GDL practices (Multicultural Programming, Global Living Learning Communities, Education Abroad, and Global Studies Courses) to desired learning outcomes (Self-Awareness, Other-Awareness, and Critical Thinking). Using an existing dataset of 1893 participants, this study examines the influence of prior participation, gender, socio-economic status, and citizenship within GDL practice types. The study findings also reveal significant differences between group members on Other Access and Critical Thinking scores. Understanding how different GDL practice types affect achievement of desired outcomes will allow GDL practitioners to seek out collaboration between departments and align programming, to allocate resources more effectively, and communicate potential outcomes based in empirical data. 
Study implications for practitioners. The research that has been done on GDL practices is typically focused on one practice type in isolation. The messy reality at our institutions, however, is that students can choose to participate in many types of GDL practices and often do participate in more than one practice, sometimes simultaneously. The organic, entangled nature of student experiences makes measuring the impact of a single experience challenging at best.

The findings of this study can offer us a few pieces of information that can inform our practices to help us collaborate between units to design, deliver, and assess GDL practices like education abroad and global studies courses. The following sections highlight findings and what they could mean for practioners.

Finding 1. Education Abroad and Global Studies practices have significantly different outcomes and attract students with significantly different scores in all three outcomes.

1. Education abroad participants began their experiences with significantly higher scores in Critical Thinking, Other Access, and Self Access than Global Studies participants.

2. Education abroad participants lost less in Critical Thinking scores than their peers in Global Studies courses.

3. Education Abroad participants also increased more in Other Access scores in comparison to Global Studies participants.

What this means for us as practitioners is that we should find ways to consider who students are before they participate in our GDL practices. When we create models of how our programs work (see handouts) student inputs should be part of those models. It 
is also possible that the experiential nature of the education abroad experience demands and has real time feedback for participants in a way that develops Critical Thinking in ways classroom practices in Global Studies does not. Regarding score losses that were seen in both studies, it is important to consider the emotional and psychological impact of encountering input that is not congruent with the way one sees the world. The stress that is caused by incongruence can lead students to withdraw into more simplistic explanations or more familiar ways of interpreting the world (Wandschneider et al, 2015). As we design our GDL practices, we should not shy away from precipitating this discomfort, however, we should ensure that adequate support for reflection and dialogue are embedded in our practices so this retreat into comfortable places is only temporary.

Finding 2. Gender predicted advantage across all outcomes for Education Abroad and in Critical Thinking for Global Studies. Additionally, income level had a negative relationship to change in Other Access scores for Education Abroad students.

1. Education Abroad students who identified as female had significantly higher scores on Critical Thinking, Other Access, and Self Access than those who identified as male.

2. Global Studies students who identified as female had significantly higher scores on Critical Thinking.

3. The higher the family income level for the Education Abroad student, the lower the change score on Other Access.

These findings, upon first glance raise, issues that few of us really want to tackle. Returning to findings from prior research, however, can be helpful. A few studies have found significant outcome differences between genders (Connor-Linton, Paige, \& Vande 
Berg, 2009; Thakral et al., 2016; Wandschneider, Pysarchik, Sternberger, \& Ma, 2015). Wandschneider and colleagues (2015) found that students who identified as female were significantly more likely to indicate interest in global learning than their male counterparts. This differential interest and willingness to participate can be seen in overall participation numbers for Education Abroad in the US (Institute of International Education, 2016). It is possible that females are then more open to the full experience of education abroad, participating in optional excursions or reflection sessions, and this increases their ability to think critically about differences and what they mean for how they see themselves. For income, it is possible that students from lower socio-economic brackets have fewer opportunities to interact with people who possess a set of values and behaviors different from their own. It would explain why their gain scores on Other Access would be greater than those of students who were able to travel or afford extracurricular activities and support in wealthier families.

\section{Next Steps}

During our session we will discuss how to approach our practices with these findings in mind. We will begin by considering our own assumptions about what is needed to produce outcomes of critical thinking and awareness of self and other. Use of a logic model will help us identify these assumptions. We will then discuss ways to identify other units in our institutions which have complementary outcome goal and strategies for approaching partners mindfully. 
Slide Presentation

\title{
Do different types of global and diversity learning practices produce different outcomes?
}

\author{
Jennifer L. Wiley, \\ University of Missouri-Columbia
}

\section{Aims and Research Questions}

1. Are there differences between the types of GDL practices in regard to changes in college students' scores on Self Access, Other Access, and Critical Thinking gains (as measured by the Beliefs, Events and Values Inventory)?

2. What are the relationships between college student characteristics, types of GDL practices and changes in Self Access, Other Access, and Critical Thinking scores (as measured by the Beliefs, Events and Values Inventory)?
- Education Abroad

- GDL LivingLearning Community

- Multicultural Programming

- Global Studies Coursework 


\section{Framework}

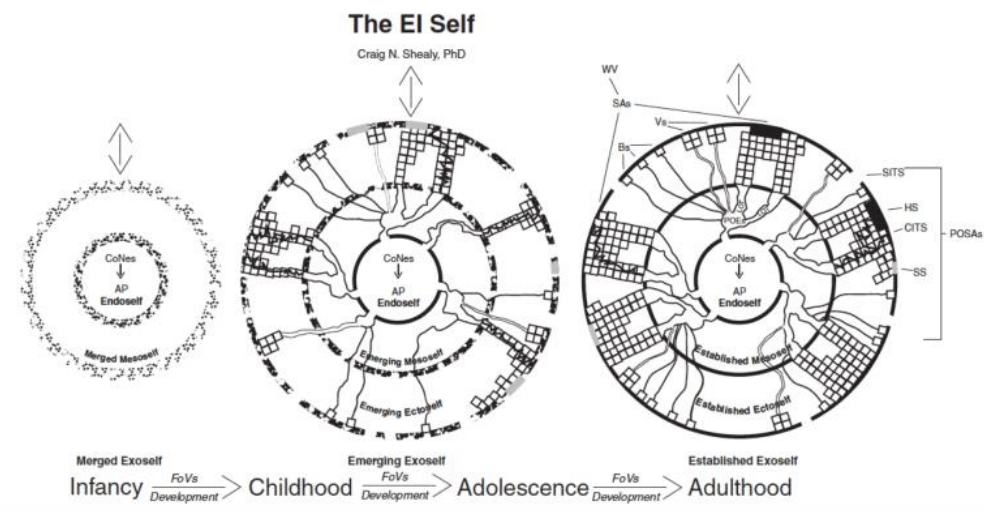

El Theory

(Shealy, 2004)

Hoggan's (2016)

Taxonomy for

Transformative

Learning

\section{Instrument}

- Beliefs, Events, and Values Inventory (BEVI) captures developmental, affective, and attributional outcomes (Shealy, 2004)

- Helps us see the process of how people form their worldview and meet their core needs

- Convergent validity demonstrated with IDI study (Reisweber,

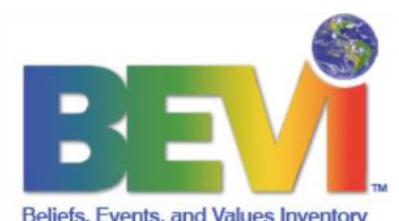
2008)

- Short version, 185 items, reliability coefficients $>.80$

- 17 scales developed through EFA (Shealy, 2004) and refined through CFA (Wandschneider et al, 2015). Collapsed into seven domains

- Three domains used: Critical Thinking, Self Access, Other Access 


\section{BEVI Domains}

Domain

\section{Critical Thinking}

Self Access

Other Access
Description of scales within each domain

Scale 7: Basic Determinism: Chooses simple explanations for phenomena, sense of fixed character

Scale 8: Socioemotional Convergence: thoughtful, determined, sees complexities in circumstances, aware of connectivity between self and larger world Scale 9: Physical Resonance: receptive to needs and feelings of own body Scale 10: Emotional Attunement: connected to own emotions; sensitive to and accepting of expressions of affect in others

Scale 11: Self Awareness: reflective, okay with complexity and difficult feelings

Scale 12: Meaning Quest: seeking balance in life; searching for meaning

Scale 13: Religious Traditionalism: sees life as mediated by God, highly committed to religious doctrine

Scale 14: Gender Traditionalism: binary in thinking about sexes and roles that are assigned to sexes. Prefers simple view of sex and gender.

Scale 15: Sociocultural Openness: open to an array of policies and practices; looks for experience of difference

\section{Variables of Interest}

\section{- GDL Practice Types-Predictor RQ1}

- Education Abroad

- Global Studies Courses

- Multicultural Programming

- Global/Diversity Living-Learning Communities

- Demographic characteristics-Predictor

RQ2

- Age

- Income

- Gender

- Nationality

- Prior program experience
- Scale scores-Outcome Variables

- Critical Thinking

- Other Access

- Self Access 


\section{Data}

Archival data from prior BEVI administrations

- Collected between September 2010 and July 2017

- 37 institutions, US and non-US institutions of higher education

- Multiple types of practices based inside and outside of the US

- After cleaning and removal of duplicates, $n=1893$

\begin{tabular}{|l|c|c|}
\hline \multicolumn{1}{|c|}{ Institution Type } & $\begin{array}{c}\text { Number of } \\
\text { Institutions }\end{array}$ & $\begin{array}{c}\text { Percent } \\
\text { of Total Participants }\end{array}$ \\
\hline Medium US Public 4 year & 5 & 18 \\
\hline Large US Public 4 year* & 8 & 57 \\
\hline Small US Private 4 Year & 5 & $<1$ \\
Non US University & 5 & 10 \\
Study Abroad Provider & 14 & 13 \\
\hline Totals & 37 & 100 \\
\hline
\end{tabular}

\section{Data: Sample Descriptives}

- Mean age at Time 1 is 20.6 years. (SD 3.8 years)

- 62 percent female

- Median income is $\$ 80,000$ with a range between $<\$ 10,000$ and $>\$ 250,000$

- 44 percent of the participants indicated prior participation in a GDL practice

- Participants were US and non-US citizens

- There were not enough observations of Multicultural Programming and LLCs in the dataset to include them in the analysis 


\section{Analysis}

- RQ1: Are there differences between the types of GDL practices regarding changes in college students' awareness of self and others and critical thinking (as measured by the Beliefs, Events and Values Inventory [BEVI])? (ANCOVA)

- Outcome: Change scores on three BEVI domain scales (Critical Thinking, Other Access, Self Access)

- Predictor: Service type (Study Abroad, Global Studies Curriculum)

- Covariate: Initial scores (Time 1) on respective domain scales

\begin{tabular}{|l|c|c|c|c|c|} 
& \multicolumn{2}{|c|}{$\begin{array}{c}\text { Education Abroad } \\
(\mathbf{n}=\mathbf{1 0 8 1})\end{array}$} & \multicolumn{2}{|c|}{$\begin{array}{c}\text { Global Studies } \\
(\mathbf{n = 8 1 2})\end{array}$} & \\
\hline & Mean & SD & Mean & SD & Difference \\
\hline Critical Thinking & 63.54 & 27.60 & 49.65 & 29.52 & $13.89^{*}$ \\
\hline Other Access & 63.78 & 19.63 & 50.53 & 21.84 & $13.25^{*}$ \\
\hline Self Access & 65.17 & 19.82 & 60.12 & 22.91 & $5.05^{*}$ \\
\hline
\end{tabular}

\section{Analysis}

- RQ2: Relationship between changes in outcome scores and participant demographics/prior experience (simple linear regressions)

- Outcome: Changes in scores on three domain scales (Critical Thinking, Other Access, Self Access)

- Predictors: Gender, Age, Income, Nationality, and Prior experience in GDL practice

- Covariate: Initial scores (Time 1) on respective domain scales

To answer RQ2, A multiple regression analysis was completed using 3 gain score outcomes (accounting for Time 1 scores) and participant characteristic predictor variables. 


\section{Grounding the Findings}

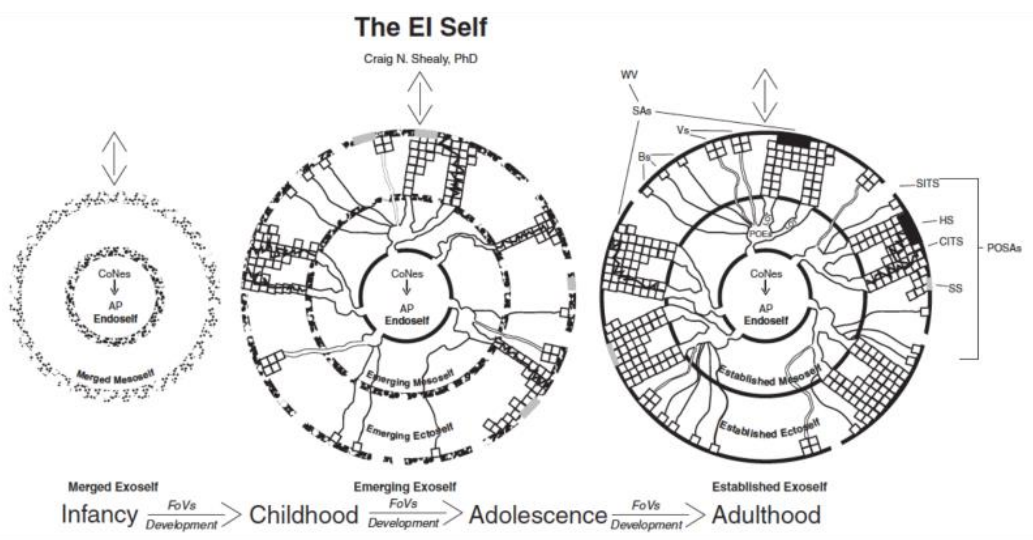

\section{Findings from Research Question 1}

- Practices differed significantly on two outcomes:

- Critical Thinking: Participants in education abroad $(m=-2.69)$ showed fewer losses than those in global studies $(m=-8.17)$ with an adjusted $R^{2}$ of 0.13

- Other Access: Participants in education abroad experience nominal positive change $(m=0.66)$, while those in global studies decreased scores $(m=-1.36)$ with an adjusted $R^{2}$ of 0.10

- Self Access: No difference between practice types 


\section{Education Abroad and Global Studies: Critical Thinking Pre-experience}

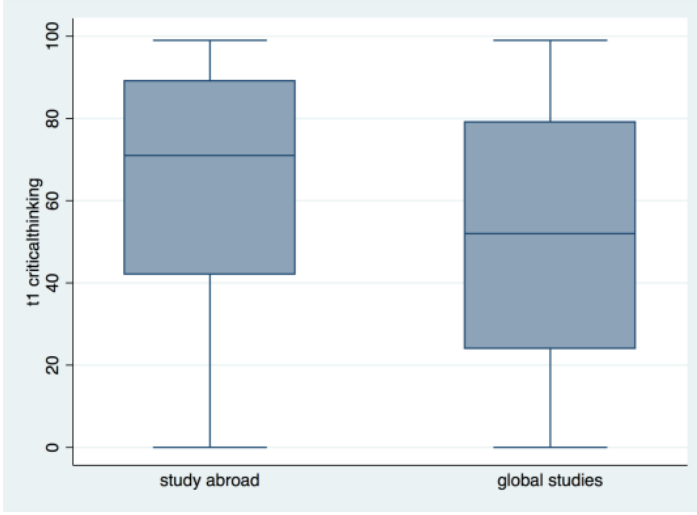

While it was true for all outcome variables, mean Time 1 scores for Education Abroad participants were significantly higher than Global Studies. These two groups have different skill sets and dispositions as they enter their GDL practices.

\section{Findings from Research Question 2}

- Gender predicted smaller negative changes in all three outcomes for female education abroad participants

- Females also showed less negative change in critical thinking for global studies programs

- Non-U.S. citizens experienced greater decreases critical thinking and other awareness in global studies programs, but unequal sample sizes call for caution in interpreting these data

- Income had an inverse relationship with Other Access (the higher the income the lower the positive change) 


\section{Implications for Practice \\ WHAT DOES IT MEAN THAT GENDER IDENTIFICATION IS PREDICTIVE OF ADVANTAGE ON ALL EDUCATION ABROAD OUTCOMES?}

- As facilitators of global learning, we need to pay attention to who students are as they participate in programming,

- Students identifying as female tend to have greater interest in global learning (Wandschneider et al, 2015) that could lead them to participate more fully in the global learning experience

- When designing experiences, differentiation of activities may be important to help male students understand the benefits of the outcomes they may not associate with career development

\section{Implications for Practice}

COLLABORATE ACROSS CAMPUS

- The differential between Critical Thinking and Other Access outcomes of Education Abroad and Global Studies were significant but effect sizes are low.

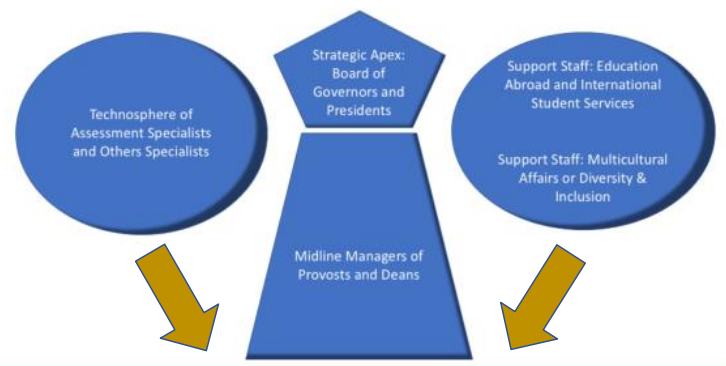




\section{Implications for Practice}

FUNDING FOR LOW SOCIO-ECONOMIC STATUS STUDENTS COULD MAKE A DIFFERENCE

- When advocating for funding support for education abroad programs, remember that students from lower family incomes can benefit more in critical thinking development

\section{Limitations}

- Non-random sample limits the generalizability of findings

- No ability to assign causality, only associations between variables

- Limitations in how to characterize demographics (e.g. US and nonUS)

- The way the data was collected limits consideration of experience duration

- No way to consider components of practices which could contribute to learning (e.g. instructor influence, quality of design, depth of experience, etc.) 


\section{Further Research}

- Include a wider array of GDL practices

- Understand factors involved in gender differences

- Consider a different statistical approach to understand potential for populations not currently participating in representative numbers, e.g., propensity score matching

- Operationalize facets of GDL experiences in order to understand interactions between who students are and the elements of the learning experience

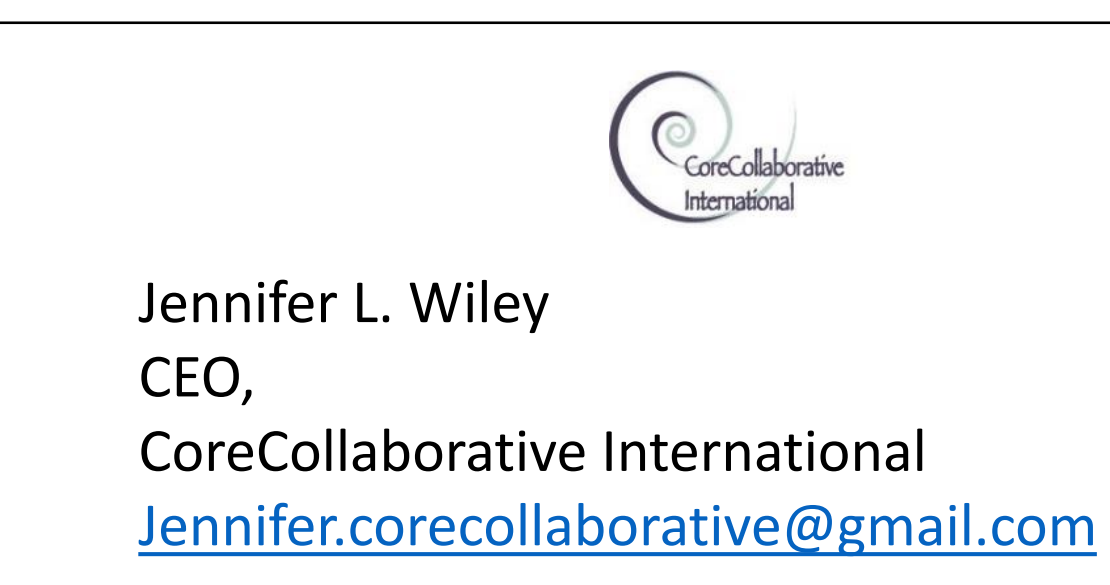




\title{
Handouts
}

\author{
Assessing Global Learning: Influence of Design and Student Demographics \\ Jennifer L. Wiley, CoreCollaborative International
}

You have probably heard someone say, "The time to think about assessment/evaluation is before you begin." The benefits of this approach are not just about best practice (though it is a best practice), it can save your organization considerable amounts of time and resources to plan ahead. For participants, it also helps to begin with outcomes. This communicates to students what your institution expects from them as well as provides you with the opportunity to understand what the students bring to and expect from the global learning experience. The questions and logic model below are intended to provide a reflective guide for you to think holistically about the learning that you are responsible for assessing.

1. What are the characteristics and components of the global learning experience (this could be as broad as a degree program or as focused as an intensive study abroad experience)?

2. What are the most important outcomes of that global learning experience? Where did those outcomes originate?

3. Why are those outcomes important to your institution?

4. What are the qualities of a student who had learned and achieved all of the outcomes your organization desired from the learning experience? What would they demonstrate after completing the experience? What would the student:

Be able to do?

Have the capacity to understand?

Come to believe?

Be able to express, say, or report?

5. What are the characteristics of the students who are attracted to your course/program/experience, e.g., gender, age, prior experience, etc? 
6. How might the students' characteristics (experiences, knowledge, skills, etc.) before participation impact the way they experience your program? How do you/do you assess these characteristics in your program design?

7. Would you expect the students you described above to already have some of these skills/dispositions/attitudes? Why or why not?

8. What evidence would you need to be confident students completed your program with the desired outcome? What evidence would your institution need? What would you do with it? 

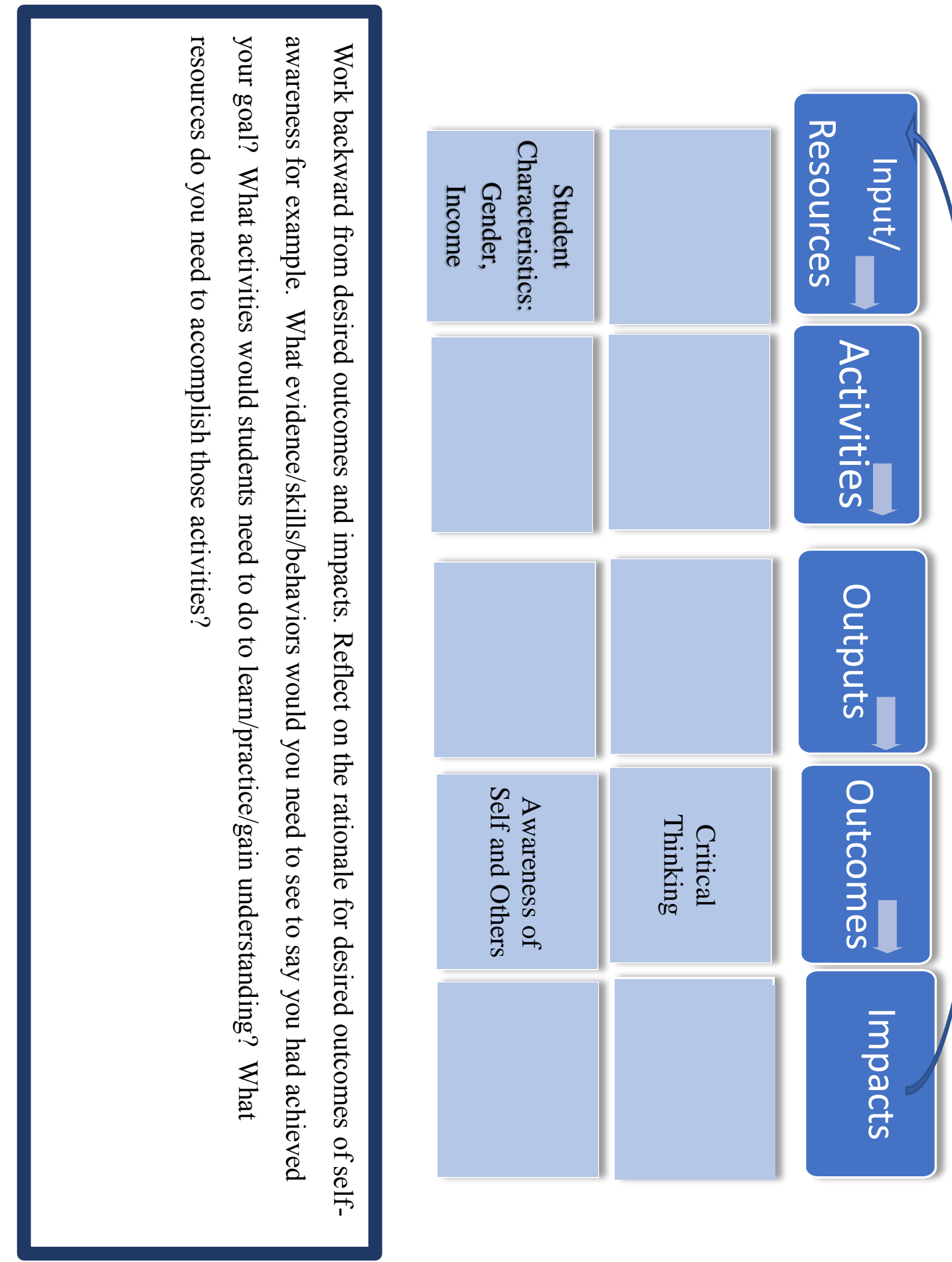

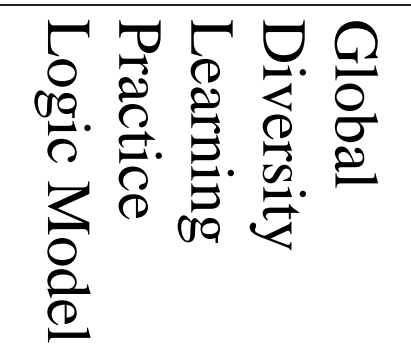




\section{SECTION FIVE \\ CONTRIBUTION TO SCHOLARSHIP}

Reader's Note: The article below will be submitted to the Journal of Transformative Education. 
Global and Diversity Learning Practices in Higher Education:

Understanding the Influence of Student Characteristics and Practice Type on 21st

Century Outcomes

Jennifer L. Wiley

University of Missouri, Doctoral Candidate

Contact Information:

Jennifer L. Wiley

1334 N. Benton Avenue

Springfield, Missouri 65802

Phone (417) 880-2430

Email: jjoneswiley@gmail.com

Previous oral presentation of the paper:

NAFSA Research Symposium, Washington, D.C. April 5, 2018 


\begin{abstract}
The ability to think critically and complexly amid novel experiences that require self- and other-awareness is something that leaders in an array of social institutions seek to develop in their communities. Global and diversity learning (GDL) practices, one category of high impact practices (Kuh, 2008), aim to increase students' awareness of self and others and imbue critical thinking skills that will help students see how their background and experiences interact with those of peers. This study aims to understand the relationship of four GDL practices (education abroad, multicultural programming, intercultural living-learning communities, and global studies coursework) to desired learning outcomes. Through completing ANCOVAs and multiple regression analyses on an existing dataset of GDL participants, this study demonstrates the influence of gender, socio-economic status, and citizenship within GDL practice types. The study findings also reveal significant differences between group members' other awareness and critical thinking capacity. Understanding how different GDL practice types affect capacity development will allow university leadership to direct collaboration between departments and align programming, to allocate resources more effectively, and communicate potential outcomes based on empirical data.
\end{abstract}




\section{Introduction}

"In terms of cultural and political matters, you are likely to be described as accepting, open, liberal, and progressive. Such ways of thinking and being can be wonderful, as you likely anticipate eagerly an encounter with ideas, approaches, circumstances, people, or societies that are new or different, and feel a sense of exhilaration when doing so." She quietly folded the paper, peered from the corner of her eye to see if anyone else was watching, pulled her arms across her body tightly, and slid down in her chair to wait for the debrief session to begin. It only took one word in her diagnostic report to create the same amount of internal conflict in Anna's mind as her combined experiences studying abroad that semester. At the beginning of the group debrief, she raised her hand, suggested that the validity of the instrument was akin to a Vanity Fair magazine quiz, and categorically rejected the idea that she was "liberal." Her group mates giggled warmly and confirmed that she was the lone "conservative," but she was unequivocally their conservative. The facilitator asked Anna and her group mates to talk about what these labels meant in both their host community and in a US context. A healthy discussion ensued, but Anna drifted into the background. Toward the end of the debrief session, the facilitator asked Anna if she felt comfortable sharing what she was thinking. Anna replied, 'I don't want to go home, because I won't fit in anymore. None of my friends or family will understand my experiences here." After studying abroad in Chile for a semester, Anna had been critically reflecting on what it would mean if her way of seeing the world had changed. She was beginning her process through cognitive disequilibrium, the process that takes place when one's understanding of the way the world functions and new experiences or information are incongruous (Mezirow, 1997; 
Piaget, 1952; Shealy, 2015). For Anna, her understanding of her own identity and political affiliation met with contrasting information she could not ignore. She felt a sense of belonging and shared some beliefs with a group of people, who prior to studying abroad, were merely 'the other.' As she began to make meaning with this new information, she became somewhat distressed. What would happen if she had changed? How would her needs for affiliation and connection be met when she went home and people noticed the changes? Could the study abroad program have been designed to better prepare her for this internal conflict? What would have been the ideal outcome for Anna, the program organizers, or the university that promoted her study abroad experience? Is this the type of outcome university administrators and faculty members are after when they talk about high impact or transformative learning practices?

\section{Background of the Study}

The ability to think critically and with complexity in the face of novel or unexpected information, like Anna's experience, that require self and emotional awareness are outcome goals that military, corporate and civic leaders are seeking to develop in their respective populations (Abbe, Herman, \& Gulik, 2007; Gardner, Gross, \& Steglitz, 2008; Kuh, 2005). Response to demand for these skills began to grow in US higher education through initiatives like the American Association of Colleges and Universities' (AAC\&U) Liberal Education and America’s Promise (LEAP) campaign, launched in 2005. Knowledge of human cultures and the natural world, intellectual and practical skills, ability to recognize responsibility for self and others, and ability to apply learning were the core learning outcomes identified through the initiative (Maki \& Schneider, 2015). What universities needed was a system of experiences that targeted 
these specific outcomes because many of these skills and understandings are not targets of conventional content courses within programs of study.

To meet US university needs for programming that would facilitate development of these targeted 21 st century skills, AAC\&U commissioned several literature reviews and studies to determine what works in higher education. Ten overarching categories of high impact practices (HIPs) emerged as the most effective mechanisms for developing 21st skills (Kuh, 2008). One category of HIPs, global and diversity learning practices (GDLs), specifically targets competencies that are needed to permit students to think critically about who they are in relationship with others. GDL practices include experiences like multicultural programming, intercultural living-learning communities, education abroad, and global studies coursework. Sandeen (2012), suggested that global and diversity learning should go beyond the traditional international focus and encourage students to think about social justice struggles in a more complex manner. It is not uncommon to see the label transformative applied to GDL practices. Scholars have posited that students should encounter differences that will lead them to disequilibrium and reflection on the origins of their worldview (Brownell \& Swaner, 2010, Sandeen, 2012).

GDL practices are complex, multi-component experiences whose individual contribution to changes in student cognitive and emotional outcomes like awareness of self and others are difficult to isolate. The internal outcomes for individual students, such as self-awareness, are less understood because the variables increase when one considers who a student is prior to the learning experience. Wandschneider and her colleagues (2015) noted that isolating the contribution of any practice toward significant change in a 
student's worldview is also particularly challenging because of its entanglement with the individual's internal variables like prior experiences. In the current, polarizing climate in the US, with many campuses grappling with how to create cohesion amid rising tension over race and national identity, it is imperative that the academic community explore how these high impact initiatives could change more students' ways of perceiving themselves and interacting with others in the world.

Having established goals for learning outcomes and a range of practices that have the potential to meet these outcomes, there are four challenges that GDL practice administrators and scholars need to address. The first is the is a need to identify a common theoretical framework for change that addresses target outcomes. A quick scan of publications related to GDL practices reveals several theories for how the practices produce change (Kilgo, 2016; Basow \& Gaugler, 2017; Lou, Paige, \& VandeBerg, 2012; Medina-Lopez-Portillo, 2004; and Wandschneider et al, 2015). While a diversity of perspectives is important, different frameworks for change result in disjointed recommendations for practitioners to use as they design transformative GDL practices. For example, recommendations that stem from Experiential Learning Theory (Kolb, 2015) might deal with changes in instructional style while recommendations from the Developmental Model of Intercultural Sensitivity (Bennett, 1998) might offer activities for students to practice frame shifting. There is an appreciable gap in frameworks that would speak to how those worldviews are created and sustained or modified. Second, the outcome variables studied in current literature focus on output measures like retention rates or grade point averages (Astin, 1993; Kuh, 2008; Provencher \& Kassel, 2017). While they are important, they do not directly measure the outcomes that a wide array of 
stakeholders value. Third, current studies look at practices in isolation making it difficult to compare the impact of one type of GDL practice in comparison with another (Acheson et al, in press). This is an important point because HIPs in general do not enjoy wide participation by all demographic groups or even a majority of students in universities and colleges (Brownell \& Swaner, 2010). Additionally, the empirical evidence to support claims for the impact of a single practice type, like education abroad, is based upon research that is subject to limitations of small sample size and over reliance on student self-report of learning (Ogden, 2015). Some GDL practices, like education abroad for example, have overall participation rates of ten percent with students of color being underrepresented among participants (Open Doors Data, 2016). Finally, evaluation of GDL practices often overlooks how differential incoming student characteristics may predict differential outcomes from the same experiences. The purpose of this study is to address these four challenges and understand which types of GDL practices are most closely related to changes in awareness of self and others and critical thinking and how the characteristics of student participants influence outcomes.

This study has implications for scholarship and practice in US higher education. Scholars and practitioners need to broaden their understanding of how and why change does or does not occur and for whom when students participate in GDL practices (Ogden, 2015). The significance of this study for practioners rests in the exploration of the interactions between types of practices and student characteristics. Knowing whether or not there are types of practices that are more effective for particular groups of students (not just from a demographic perspective) would permit program advisors and developers to counsel students more effectively or prepare students for 
experiences more accurately. It could also permit leaders of institutions to target a single student learning outcome more effectively.

The research question driving this study is: What are the relationships between college student characteristics, types of GDL practices and changes in awareness of self and others, and critical thinking scores (as measured by the Beliefs, Events and Values Inventory)?

\section{Theoretical Framework}

The snapshot from a sample GDL practice at the beginning of the article provides a context for the framework of this study. When Anna was confronted with information that contradicted her understanding of who she was and how she viewed others, there was an educational space for mindful support and intervention. Which factors lead a student to change or resist changing? When a student does undergo change, what must change in order for it to be labeled a transformative or high impact change? The following section will outline the theories that create the foundation for this study, keeping Anna's experience present and a reminder of the applied context of learning.

Perhaps because of their many contexts and interdisciplinary nature, high impact practices, like GDL practices, do not share a common theoretical framework. Scholars concerned with HIPs have applied a variety of theoretical frames from Critical Theory (Kilgo, 2016), to the Developmental Model of Intercultural Sensitivity (Connor-Linton et al., 2009; Engle \& Engle, 2004; Stebleton, Soria, \& Cherney, 2013) to Global Citizenship (Friedman, Haverkate, Oomen, Park, \& Sklad, 2015). Authors may bring their own disciplinary background and training to explain why they expect certain outcomes from these types of practices. 
Two complementary theoretical frameworks are put to use here to explain how GDL practices could facilitate change in students' awareness of self and other and critical thinking. The first theoretical frame, Equilintegration (EI) Theory (Shealy, 2004, 2006, 2015) provides the study with a foundation for how people initially develop and maintain or change the what they believe about themselves and the world around them. The second theoretical frame, Transformative Learning Theory (Hoggan, 2016; Mezirow, 1997) is used to describe the layers within the individual that change which provide a bridge to pedagogy that can inform GDL practices.

The first, EI Theory (Shealy, 2004, 2006, 2015), helps explain how students enter a GDL practice, from a psychological readiness perspective, and why they may or may not resist learning or change. EI Theory (Shealy, 2004, 2006, 2015) provides the framework for understanding how students develop the way they see themselves and the worldviews that they bring to interventions like GDL practices. EI Theory draws connections between the many facets of who people are, how their needs are met, and how input from their environment shapes their system of beliefs and values, or worldview. It provides a framework for understanding why interventions may have different outcomes for different students. Through this framework, we begin to understand how students come to learning experiences with a particular worldview, or system of acquiring beliefs and values. Individuals are composed of beliefs and values that are available to them as they develop (Shealy, 2004; Vygotsky, 1997). Being unaware that they have a worldview, through lack of availability to contrasting views, may prevent students from being able to learn or change. Through novel experiences, that are not consonant with their prior experiences, individuals may gain awareness of 
their own beliefs and values, and those of others. For true change in awareness or critical thinking to occur, some sort of intervention may be necessary to act as a catalyst (Dabrowski, 1964; Dirkx, 1998; Dweck, 2008; Mezirow, 1997). This can disturb a sense of balance the self has created regarding how the world makes sense (Shealy, 2015), therefore, GDL practices should be designed with support for reflection.

The second, Transformative Learning Theory (Mezirow, 1997) provides a bridge to the pedagogical design of GDL practices. Transformative Learning Theory describes what specific aspects of a student should change in order to claim an experience has sustained, high impact on student learning outcomes. The Transformative Learning framework established by Hoggan (2016) could allow educators to design GDL practices that would target change in those specific elements of who students are and how they see, or do not see, themselves and the world.

EI Theory (Shealy, 2004, 2006, 2015) provides the context for how Anna formed the beliefs about herself and 'liberal' others that she brought to the learning experience. It also explains the interconnectedness of core needs and belief formation that can provide understandings of why learning or change is resisted at times. There is a gap, however, between knowing how a student's worldview is formed and the learning environment. Transformative Learning Theory (Mezirow, 1998) can provide the bridge between the two contexts by identifying components of the student that should change in order for the experience to be identified as high impact. This allows the educator to systematically design learning experiences and environments that could support change in each of these components. Hoggan's (2016) taxonomy to describe essential components of transformation outlines six fundamental components: (a) a change in 
worldview, (b) changes in ways of knowing, (c) changes in cognitive ability, (d) increased awareness of self and other, (e) increased mindful awareness of subconscious dialogue, and (f) changes in action.

Using Hoggan's (2016) framework, a well-designed GDL practice should seek to address change in each of these six components of a transformative learning experience. For the scope and purposes of this study, three components will serve as dependent variables as operationalized by the Beliefs, Events, and Values Inventory (BEVI; Shealy, 2004): (a) worldview change, operationalized by the Other Access domain of the BEVI; (b) increased self-awareness operationalized by the Self Access domain of the BEVI; and (c) changes in cognitive ability operationalized by the Critical Thinking domain of the BEVI. Changes in action, ways of knowing and subconscious dialogue are not captured in the available dataset but should be considered for future study.

In an effort to elucidate the relationships between the two theories, pedagogical approach, and assessment instrument used in this study, Table 5 provides a cross references for concepts central to EI Theory (Shealy, 2004, 2006, 2015), Transformative Learning Theory (Mezirow, 1998; Hoggan, 2016), High Impact practice literature (Kuh, 2005), and the component of the instrument used in this study, the Beliefs, Events, and Values Inventory (BEVI) created by Craig Shealy (2004). A discussion of the Transformative Learning components operationalized in this study follow Table 5. 
Table 5

Alignment of Theoretical Framework to Practice Measures

\begin{tabular}{|c|c|c|c|}
\hline $\begin{array}{l}\text { EI Theory applied } \\
\text { (Shealy, 2016) }\end{array}$ & $\begin{array}{l}\text { Transformative } \\
\text { Learning Theory } \\
\text { elements } \\
\text { (Hoggan, 2016) }\end{array}$ & $\begin{array}{l}\text { High impact } \\
\text { practice outcomes } \\
\text { (Maki \& } \\
\text { Schneider, 2015; } \\
\text { Kuh, 2008) }\end{array}$ & $\begin{array}{l}\text { BEVI-Short version: } \\
\text { Domain alignment } \\
\text { (Acheson et al., in } \\
\text { press) }\end{array}$ \\
\hline $\begin{array}{l}\text { Worldviews form } \\
\text { based upon the } \\
\text { interaction of } \\
\text { social context and } \\
\text { how formative } \\
\text { needs were met }\end{array}$ & $\begin{array}{l}\text { Worldview } \\
\text { shifts }\end{array}$ & $\begin{array}{l}\text { Global knowledge } \\
\text { in relation to self }\end{array}$ & $\begin{array}{l}\text { Other Access Domain: } \\
\text { open regarding ways of } \\
\text { functioning in social } \\
\text { context, capacity for } \\
\text { dealing with the } \\
\text { existential and non- } \\
\text { corporeal, open }\end{array}$ \\
\hline $\begin{array}{l}\text { Changes in } \\
\text { worldview come } \\
\text { through } \\
\text { disequilibrium } \\
\text { experiences that } \\
\text { challenge } \\
\text { assumptions }\end{array}$ & $\begin{array}{l}\text { Changes in } \\
\text { underlying } \\
\text { assumptions }\end{array}$ & & $\begin{array}{l}\text { understanding of } \\
\text { gender in social } \\
\text { contexts, and interest } \\
\text { in interactions with } \\
\text { unfamiliar contexts and } \\
\text { personal backgrounds }\end{array}$ \\
\hline $\begin{array}{l}\text { Awareness of the } \\
\text { internal system for } \\
\text { belief } \\
\text { development }\end{array}$ & $\begin{array}{l}\text { Ways of } \\
\text { knowing are } \\
\text { more open, } \\
\text { discriminating, } \\
\text { inclusive; }\end{array}$ & $\begin{array}{l}\text { Intellectual skills, } \\
\text { e.g., critical } \\
\text { thinking, } \\
\text { teamwork, problem } \\
\text { solving (Kuh, } \\
2008 \text { ) }\end{array}$ & $\begin{array}{l}\text { Critical Thinking } \\
\text { Domain: capacity for } \\
\text { complex explanation of } \\
\text { differences, awareness } \\
\text { of larger world and } \\
\text { entangled nature of }\end{array}$ \\
\hline $\begin{array}{l}\text { Understanding of } \\
\text { internal dialogue } \\
\text { and processes }\end{array}$ & $\begin{array}{l}\text { Increases in } \\
\text { cognitive } \\
\text { abilities }\end{array}$ & & interactions with others \\
\hline
\end{tabular}

(continued) 
Table 5.1 (continued)

\begin{tabular}{|c|c|c|c|}
\hline $\begin{array}{l}\text { EI Theory applied } \\
\text { (Shealy, 2016) }\end{array}$ & $\begin{array}{l}\text { Transformative } \\
\text { Learning Theory } \\
\text { elements } \\
\text { (Hoggan, 2016) }\end{array}$ & $\begin{array}{l}\text { High impact } \\
\text { practice outcomes } \\
\text { (Maki \& } \\
\text { Schneider, 2015; } \\
\text { Kuh, 2008) }\end{array}$ & $\begin{array}{l}\text { BEVI-Short version: } \\
\text { Domain alignment } \\
\text { (Acheson et al., in } \\
\text { press) }\end{array}$ \\
\hline $\begin{array}{l}\text { To change, people } \\
\text { must have } \\
\text { awareness of core } \\
\text { needs, (affective, } \\
\text { affiliative) and } \\
\text { personal beliefs }\end{array}$ & $\begin{array}{l}\text { Ways of being, } \\
\text { more } \\
\text { emotionally in } \\
\text { tune, } \\
\text { independent, } \\
\text { aware }\end{array}$ & $\begin{array}{l}\text { Personal and Social } \\
\text { Responsibility, } \\
\text { e.g., intercultural } \\
\text { awareness, civic } \\
\text { knowledge and } \\
\text { action (Kuh, 2008); } \\
\text { Maki \& Schneider, } \\
\text { 2015) }\end{array}$ & $\begin{array}{l}\text { Self Access Domain: } \\
\text { cares for human } \\
\text { condition; tolerates } \\
\text { difficult feelings; } \\
\text { resilient; } \\
\text { receptive to corporeal } \\
\text { needs; } \\
\text { sensitive, social, values } \\
\text { the expression of affect }\end{array}$ \\
\hline \multirow[t]{2}{*}{$\begin{array}{l}\text { As awareness of } \\
\text { core needs and } \\
\text { their relationship to } \\
\text { beliefs becomes } \\
\text { apparent, people } \\
\text { can see themselves } \\
\text { independent of } \\
\text { their social context }\end{array}$} & $\begin{array}{l}\text { Changes in sense } \\
\text { of identity, } \\
\text { efficacy, } \\
\text { empowerment }\end{array}$ & $\begin{array}{l}\text { Personal and Social } \\
\text { Responsibility, } \\
\text { e.g., intercultural } \\
\text { awareness, civic } \\
\text { knowledge and } \\
\text { action (Kuh, 2008) }\end{array}$ & $\begin{array}{l}\text { Self Access Domain: } \\
\text { introspective; accepts } \\
\text { complexity of self; } \\
\text { cares for human } \\
\text { condition, open } \\
\text { regarding practices in } \\
\text { social context }\end{array}$ \\
\hline & $\begin{array}{l}\text { Behavior aligns } \\
\text { with changes in } \\
\text { worldview } \\
\text { framework }\end{array}$ & $\begin{array}{l}\text { Applied learning, } \\
\text { civic engagement, } \\
\text { ethical action } \\
(2008)\end{array}$ & \\
\hline
\end{tabular}

Note. Modified from Acheson, et al. (In press).

\section{Literature Review}

The push for global learning is, to some, an indication of that universities are shifting from a focus on instruction in a singular classroom context to one of learning across an array of authentic environments (Green, 2013; Kahn \& Agnew, 2017). GDL programs expand past the traditional study abroad boundary. They support students as they encounter differences that will make them uncomfortable or question their 
worldview. These types of programs should have content that encourages students to think about social justice struggles (Sandeen, 2012).

While the push toward GDL programs is widespread, agreement on definitions of global and diversity learning is not (Hovland, 2014). Efforts to promote a shared definition have resulted in the development of a global learning VALUE rubric to assist universities as they seek to align learning outcomes with institutional visions (Hovland, 2014; Kahn \& Agnew, 2017; Whitehead, 2015). The word global was intentionally used to include an emphasis on sociocultural contexts that are not constrained by political boundaries. It also allows for learning outcomes that focus on the entangled interactions of multi-layered local contexts (Hovland, 2014).

Outcomes commonly associated with GDL programs range from increases in selfawareness and other-awareness (DeTurk, 2006; Lou et al., 2012; Ogden, 2006; Wandschneider, Pysarchik, Sternberger, \& Ma, 2015) to increases in critical, complex, and creative thinking (Galinsky \& Maddux, 2009; Grunzweig \& Reinhart, 2002; Hammer, 2009; Law, 2014; Lou et al., 2012; Stebleton et al., 2013; Wandschneider et al., 2015). Despite some associations, there is still agreement that the connections between these program types and outcomes are tenuous (Kuh. et al., 2015; Lou et al., 2012; Wandschneider et al., 2015). Additionally, the body of literature that would help the field understand the linkage between what the students bring to experiences and the specific types of GDL practices is quite small (Salisbury \& Sobania, 2015; Wandschneider et al., 2015). Understanding the interactions between GDL practices types and outcomes is critical in order to be able to create sustainable programs that produce predictable outcomes. 
The types of outcomes that have conventionally been captured from GDL practices are similar to those from the wider array of HIPs. Several scholars have noted that the traditional approaches to assessing these types of practices that include analysis of participation rates, GPA changes, or persistence to graduation rates are no longer adequate to warrant the label of 'high impact' (Brownell \& Swaner, 2010; Hovland, 2014; Kuh \& O’Donnell, 2013). Moving away from participation outcomes, employers' and democracy advocates' interests in 21 st century skills and global competencies have put pressure on universities to demonstrate that they are producing students with capacities like self-awareness, other-awareness, and critical thinking (Connell, 2016; Hart Research Associates, 2016; Sandeen, 2012; Whitehead, 2015; Wilson-Mulnix, 2012).

Shifting away from variables that are easily accessed, like GPA or participation rates, has posed a problem. As Whitehead (2016) points out, there is no single measure for capturing outcomes of GDL practices. Therefore, it becomes challenging to compare results of studies because each measure conceptualizes outcomes in a slightly different manner. For example, Conner-Linton, Paige, and VandeBerg (2009) studied education abroad programs and focused on intercultural awareness outcomes. Within intercultural awareness, there are components of self-awareness and other awareness. Deardorff (2009) points out how these facets of intercultural awareness are essential for one to possess in order to have successful interactions with people from diverse backgrounds. However, because the constructs, instruments, and frameworks used in various studies of GDL practices differ, it is a challenge to corroborate findings between studies. Additionally, there are multiple approaches to measurement of awareness of self and other and critical thinking. Several studies that have analyzed outcomes of GDL 
programming focused on indirect measures of awareness of self and other and critical thinking (Braskamp, Braskamp, \& Merrill, 2009; Kilgo, 2016; Kuh \& O’Donnell, 2013). These studies relied upon student perceptions of their awareness and critical thinking capacities. From an EI Theory (Shealy, 2004, 2006, 2015) perspective, it is not ideal to rely upon the self to assess capacities that it cannot explicitly or consistently conceptualize or identify. GDL practice studies that use direct measures of changes to the self (Connor-Linton et al., 2009; Iyer, 2013; Kilgo et al., 2015; Wandschneider et al., 2015) do not always use the same language for constructs that involve self-awareness or other-awareness, as in the example above regarding intercultural awareness. However, because there is not a large number of studies on GDL practices of any type focused on awareness of self and others or critical thinking; it is necessary to encompass many approaches and related conceptualizations of the three outcomes involved in this study. In the section which follows, there is a discussion of prior GDL practice research that addresses one or more outcomes associated with this study. It is important to note that the author could not find any publications that considered the contribution of GDL practice type to the outcomes of awareness of self and others and critical thinking. Therefore, the section below is organized by outcome and contains studies that considered single practice types.

Going back to the theoretical framework, Shealy (2015) points out that characteristics and formative backgrounds of learners should be kept in mind as administrators and faculty design the learning experience. Prior studies (Connor-Linton et al., 2009; Kilgo, Ezell-Sheets, \& Pascarella, 2015; Pike, Kuh, \& McCormick, 2011; Wandschneider et al., 2015) have demonstrated that characteristics such as gender, 
ethnicity, socio-economic status, and prior participation in GDL practices have explanatory value in student learning outcomes related to GDL practices.

\section{Methodology}

\section{Measure}

There was one instrument used to collect data in this study. The Beliefs, Events and Values Inventory (BEVI) is a set of psychometric scales developed by Craig Shealy (2004). The BEVI is "designed to identify and predict a variety of developmental, affective, and attributional processes and outcomes that are integral to EI Theory" (Shealy, 2004, p. 1075). The instrument's development began in the early 1990s and has undergone consistent review to maintain validity and reliability through Confirmatory Factor Analyses, expert review, and concurrent validity studies (Shealy, 2015).

Reliability and validity for this instrument are robust. Reliability coefficients for each of the scales have been recorded at above 0.80 and 0.90 (Shealy, 2015). Researchers have also indicated validity of the BEVI due to its ability to predict group membership across demographic variables (Hayes, 2001; Reisweber, 2008).

The short version of the BEVI, used to collect all of the data in this study, contains 185 items. It includes the following components (a) an extensive, modifiable set of demographic questions, (b) life history questions, (c) two validity scales, (d) seventeen psychometric scales, and (e) three qualitative items designed to collect participant reflections on their experiences. The short version is the primary version utilized by most institutions delivering GDL programs (Shealy, 2015).

Scores are calculated for each of the seventeen psychometric scales in the short version. Additionally, scales that are closely correlated are further organized under seven 
overarching domains for which composite scores are available. This study used composite scores from three BEVI domains: (a) Self Access, (b) Other Access, and (c) Critical Thinking. These three domains, which represent eleven of the seventeen subscales, were utilized as outcome variables for the purpose of this study. The composition of the domains and scales of the BEVI are included in Table 6. The domains in bold font were used in this study.

Table 6

Domains and Scales of the BEVI

\begin{tabular}{|c|c|}
\hline Domain & Description of scales within each domain \\
\hline Validity Scales & $\begin{array}{l}\text { Consistency: captures consistency of response to } \\
\text { differently worded items that measure the same } \\
\text { construct } \\
\text { Congruency: degree to which responses follow } \\
\text { statistically expected patterns }\end{array}$ \\
\hline $\begin{array}{l}\text { Formative } \\
\text { Variables }\end{array}$ & $\begin{array}{l}\text { Demographic: background items such as gender, } \\
\text { economic status, age, ethnicity, etc. } \\
\text { Scale 1: Negative Life Events (conflict in family, trouble } \\
\text { as child, etc.) }\end{array}$ \\
\hline $\begin{array}{l}\text { Fulfillment of } \\
\text { Core Needs }\end{array}$ & $\begin{array}{l}\text { Scale 2: Needs Closure: unusual explanations for why } \\
\text { things work as they do. Lack of connection to core needs } \\
\text { in self or others } \\
\text { Scale 3: Needs Fulfillment: Open to needs of self and } \\
\text { others } \\
\text { Scale 4: Identity Diffusion: Difficult crisis of identity; } \\
\text { no sense of control over life outcomes }\end{array}$ \\
\hline $\begin{array}{l}\text { Tolerance for } \\
\text { Disequilibrium }\end{array}$ & $\begin{array}{l}\text { Scale 5: Basic Openness: Ability to be open with self } \\
\text { and others about thoughts, feelings, and needs } \\
\text { Scale 6: Self Certitude: does not have the capacity for } \\
\text { deep analysis, strong sense of will }\end{array}$ \\
\hline
\end{tabular}

(continued) 
Table 6 (continued)

Domain

Description of scales within each domain

\section{Critical \\ Thinking}

Scale 7: Basic Determinism: Chooses simple explanations for phenomena, sense of fixed character Scale 8: Socioemotional Convergence: thoughtful, determined, sees complexities in circumstances, aware of connectivity between self and larger world

Self Access

Scale 9: Physical Resonance: receptive to needs and feelings of own body

Scale 10: Emotional Attunement: connected to own emotions; sensitive to and accepting of expressions of affect in others

Scale 11: Self Awareness: reflective, okay with complexity and difficult feelings

Scale 12: Meaning Quest: seeking balance in life; searching for meaning

Other Access

Scale 13: Religions Traditionalism: sees life as mediated by God, highly committed to religious doctrine Scale 14: Gender Traditionalism: binary in thinking about sexes and roles that are assigned to sexes. Prefers simple view of sex and gender.

Scale 15: Sociocultural Openness: open to an array of policies and practices; looks for experience of difference

Global Access

Scale 16: Ecological Resonance: highly committed to environmental sustainability

Scale 17: Global Resonance: desire to learn about different cultures, share experience with others from differing culture groups

Note. Domains in bold are those examined in the study. 


\section{Study Design}

This study involved pre-existing data from university students who completed the BEVI between 2007-2017. The largest subset of participants was from large, public 4year universities in the US, however, a range of educational institution types and locations were represented in the dataset. These include public and private liberal arts universities, education abroad program providers, as well as medium and small 4-year universities in the US and overseas. GDL practice types that were targeted include (a) education abroad programs for both US citizens and non-US citizens, (b) global/international/multicultural living-learning communities, (c) global studies courses, and (d) multicultural programming. Detailed definitions of practice types are listed in Table 7.

Outcome variables of awareness of self and others and critical thinking were operationalized by three domain scores from the BEVI: (a) Self Access, (b) Other Access, (c) Critical Thinking. In addition to these three BEVI gain scores from student participant archival data, background information on age, gender, income, citizenship, and prior participation in a GDL practice were available. The 1,893 participants are university students ages 17-28 who have voluntarily chosen to participate in a GDL practice. 
Table 7

Descriptions of Global and Diversity Program Types

GDL Practice Type

Description

For the purpose of this study, these types of experiences include US or international students traveling to a country they do not consider their country of origin to

Education Abroad Program

Global/International/ Multicultural Living-Learning Community study, complete an internship, participate in service learning, or participate in research. These experiences can range from one week to one year in duration and may be facilitated by either their home or host institution or a third-party provider of experiences.

This type of practice involves students from differing backgrounds living together and participating in coursework or other programming that brings the group of students together to interact. An example of this type of practice would be residence halls that are designated as foreign language learning spaces where a target language other than English is spoken. Both students who are learning and native speakers of the language live in the same hall.

Global Studies Course
This type of GDL practice is typically a credit-bearing course that facilitates learning about global systems and social issues from a variety of places around the world. While this type of course is typically part of a degree program for credit, it is not the defining feature of the practice. The integrated content connecting systems that impact multiple world regions is the critical feature of global study practices.
Multicultural Programming
This type of practice would include intergroup dialogue programs, multicultural fairs or films, special lectures or concerts targeting exposure to culture and traditions outside of the participants' own culture and traditions. 


\section{Data}

Archival data was drawn from multiple BEVI administrations across a range of institutions in the US and abroad. The largest subset of participants came from large, public, 4-year US universities. Data included scores on individual scales and domains of the BEVI, as well as demographic and background information on the testers. Duplicate records and incomplete administrations were detected and omitted. Two-tailed $t$-tests were used to explore systematic differences between the excluded observations and the remaining data on demographic predictors, with no statistically significant results. Sample demographics are listed in Table 8.

Practice types were identified, isolated, and coded through BEVI data and consultation with practice administrators and the author of the BEVI. While four GDL practice types were present in the data, only two (Education Abroad, $n=1081$; Global Studies courses, $n=812$ ) had sufficient observations to be included in the analysis. As a result, the analyses for this study were conducted on two practice types and 1,893 observations. 
Table 8

Sample Demographic Frequencies

\begin{tabular}{|c|c|c|c|}
\hline Variable Name & Level & $N$ & $\%$ \\
\hline \multirow[t]{2}{*}{ Gender } & Female & 1153 & $61 \%$ \\
\hline & Male & 727 & $39 \%$ \\
\hline \multirow[t]{4}{*}{ Age } & $<18$ & 15 & $1 \%$ \\
\hline & $19-22$ & 1265 & $73 \%$ \\
\hline & $23-25$ & 123 & $7 \%$ \\
\hline & 26 and over & 96 & $7 \%$ \\
\hline \multirow[t]{3}{*}{ Income } & $0-3$ & 561 & $30 \%$ \\
\hline & $4-7$ & 857 & $45 \%$ \\
\hline & $8-11$ & 475 & $25 \%$ \\
\hline \multirow[t]{2}{*}{ Citizenship } & Domestic & 1850 & $98 \%$ \\
\hline & International & 43 & $2 \%$ \\
\hline \multirow[t]{4}{*}{ Prior experience } & Education Abroad & 319 & $17 \%$ \\
\hline & Global Studies & 319 & $17 \%$ \\
\hline & Multicultural & 636 & $34 \%$ \\
\hline & LLC & 141 & $7 \%$ \\
\hline
\end{tabular}

\section{Analysis}

The research question was approached via a series of linear regressions, using gain scores on each of the three outcomes as the dependent variables and focusing on prior transformative experience and individual demographic traits as predictors. Analyses 
were conducted for each of the two service types of interest, and score at Time 1 was controlled for throughout by inclusion in the model as a second predictor.

In order to understand how much variance in outcome could be attributed to practice type and student characteristics, a set of block, forced entry multiple linear regression analyses were conducted. For each combination of service type (Education Abroad and Global Studies) and outcome variable (critical thinking, other-awareness, and self-awareness), a multiple linear regression was conducted for each predictor variable (three possible prior experiences, income, age, gender, and international status), for a total of 42 comparisons. Multiple linear coefficients are provided in Table 10 and 11, with indicators of significance.

Multiple Comparisons using Benjamini and Hochberg. The repeated analysis of a dataset under multiple comparisons invites inflated Type I error. While the traditional method to account for this phenomenon is to apply a Bonferroni correction, dividing the targeted $\alpha$ by the number of comparisons, Benjamini and Hochberg (1995) suggest this is unnecessarily conservative. Both corrections are indicated in the significance statistics in Tables 10 and 11.

\section{Results}

As anticipated, initial scores varied statistically significantly $(p<0.05)$ between the two practice types on all outcomes, demonstrating the importance of the inclusion of Time 1 score as a covariate in all analyses. Starting mean scores and standard deviations on all outcomes are listed in Table 9. Participants in Education Abroad practices started their experiences with greater measured capacity than participants in Global Studies. 
Table 9

Mean Outcome Scores at Time 1 Administration by Practice Type

\begin{tabular}{lllllc}
\hline & \multicolumn{2}{c}{$\begin{array}{c}\text { Education Abroad } \\
(n=1081)\end{array}$} & \multicolumn{2}{c}{$\begin{array}{c}\text { Global Studies } \\
(n=812)\end{array}$} & \\
\hline & Mean & SD & Mean & SD & Difference \\
\hline Critical Thinking & 63.54 & 27.60 & 49.65 & 29.52 & $13.89 *$ \\
Other Access & 63.78 & 19.63 & 50.53 & 21.84 & $13.25^{*}$ \\
Self Access & 65.17 & 19.82 & 60.12 & 22.91 & $5.05^{*}$ \\
\hline
\end{tabular}

*sig. $p<0.05 / 3$

Multiple linear block, forced entry regressions were run to understand the effect of GDL practice type and student characteristics on outcome gains in Self Access, Other Access and Critical Thinking. To assess linearity, a series of scatterplots of outcome scores against student characteristics for each practice type with superimposed regression line was plotted. Visual inspection of these two plots indicated a linear relationship between the variables. There was homoscedasticity and normality of the residuals.

The regression analyses revealed several significant differences in outcome gain scores based on student characteristics. There was one strong pattern that appeared in the student characteristic of gender; otherwise, findings, while significant, were scattered between characteristics and practice types. A summary of the findings appears in Tables 10 and 11.

Finding 1. For participants in Education Abroad, income had a statistically significant, inverse relationship to changes in Other Access. A significant regression was found $(F(10,1078)=68.5, p<0.001)$, with an $R^{2}$ of 0.11 . Other Access gains decreased by 0.43 points for each increase in participant income category.

Finding 2. Citizenship was a significant predictor of change in Critical Thinking and Other Awareness outcomes for participants in Global Studies courses. For Critical 
Thinking gains, a significant regression was found $(F(2,809)=77.4, p<0.001)$, with an $R^{2}$ of 0.16 . Critical Thinking gains decreased by 13.13 points in the context of non-US citizen status. Similarly, for Other Access gains, a significant regression was found $(F(2$, $809)=52.8, p<0.001)$, with an $R^{2}$ of 0.12 . Other Access gains decreased by 8.15 points in the context of international status. However, unbalanced cell sizes warrant caution on this finding, as domestic participants $(n=1830)$ far outnumbered international participants $(n=49)$.

Finding 3. Prior participation in other GDL practice types did not predict differences in any of the three outcome scores. No statistically significant regressions were found.

Finding 4. Gender was a significant predictor of advantage across multiple outcomes and in both program types. For Critical Thinking gains, a significant regression was found in Education Abroad students $(F(2,1067)=87.0, p<0.001)$, with an $R^{2}$ of 0.14 . For Education Abroad participants, Critical Thinking gains were 7.57 points higher in female students than in males.

Similarly, in Global Studies participants, a significant regression was found $(F(2$, $807)=79.4, p<0.001$ ), with an $R^{2}$ of 0.16. Critical Thinking gains for females in this population were 5.24 points higher than their male peers.

For Other Access gains, a significant regression was found in Education Abroad students $(F(2,1067)=66.7, p<0.001)$, with an $R^{2}$ of 0.11 . For Education Abroad participants, Other Access gains were 2.91 points higher in female students than in males. No significant regression was found for Global Studies participants. 
For Self Access gains, a significant regression was found in Education Abroad students $(F(2,1067)=86.3, p<0.001)$, with an $R^{2}$ of 0.14 . For Education Abroad participants, Self Access gains were 2.70 points higher in female students than in males. No significant regression was found for Global Studies participants. 
Table 10

Summary of Block Regression Analysis for Variables Predicting Education Abroad Outcomes $(N=1081)$

\begin{tabular}{|c|c|c|c|c|c|c|c|c|c|c|c|c|}
\hline \multirow[b]{2}{*}{ Variable } & \multicolumn{4}{|c|}{ Critical Thinking } & \multicolumn{4}{|c|}{ Other Access } & \multicolumn{4}{|c|}{ Self Access } \\
\hline & $B$ & $S E(B)$ & $\beta$ & $R^{2}$ & $B$ & $S E(B)$ & $\beta$ & $R^{2}$ & $B$ & $S E(B)$ & $\beta$ & $R^{2}$ \\
\hline Income & -.61 & .24 & -.33 & .13 & $-.43^{* *}$ & .13 & $-.09^{* *}$ & .11 & -.19 & .14 & -.04 & .13 \\
\hline Gender & $7.57^{* *}$ & 1.60 & $.14^{* *}$ & .14 & $2.91^{*}$ & .92 & $.09^{*}$ & .11 & $2.70^{*}$ & 1.02 & $.08^{*}$ & .14 \\
\hline Age & 0.12 & .18 & .018 & .12 & .15 & .11 & .04 & .10 & -0.01 & .11 & -.00 & .13 \\
\hline Citizenship & -1.12 & 5.48 & -.58 & .12 & -4.91 & 3.18 & -.04 & .11 & 1.31 & 3.42 & .01 & .13 \\
\hline P. Exp. EA & & & & & & & & & & & & \\
\hline P. Exp. MP & 2.84 & 1.61 & .06 & .13 & 1.76 & .94 & .06 & .12 & 1.35 & 1.00 & .04 & .14 \\
\hline P. Exp. GS & 2.65 & 1.98 & .04 & & 1.84 & 1.15 & .05 & & 1.77 & 1.24 & .05 & \\
\hline P. Exp. GLLC & 1.03 & 2.59 & .01 & & 2.69 & 1.50 & .06 & & -.31 & 1.62 & -.01 & \\
\hline
\end{tabular}

Note: *sig. $p<\alpha=.05$, correcting for multiple comparisons under Benjamini \& Hochberg $* *$ sig. $p<0.05 / 42$.

P. Exp. $=$ Prior Experience, EA = Education Abroad, MP = Multicultural Programming, GS $=$ Global Studies coursework, GLLC $=$ Global LivingLearning Community 
Table 11

Summary of Block Regression Analysis for Variables Predicting Global Studies Outcomes $(N=812)$

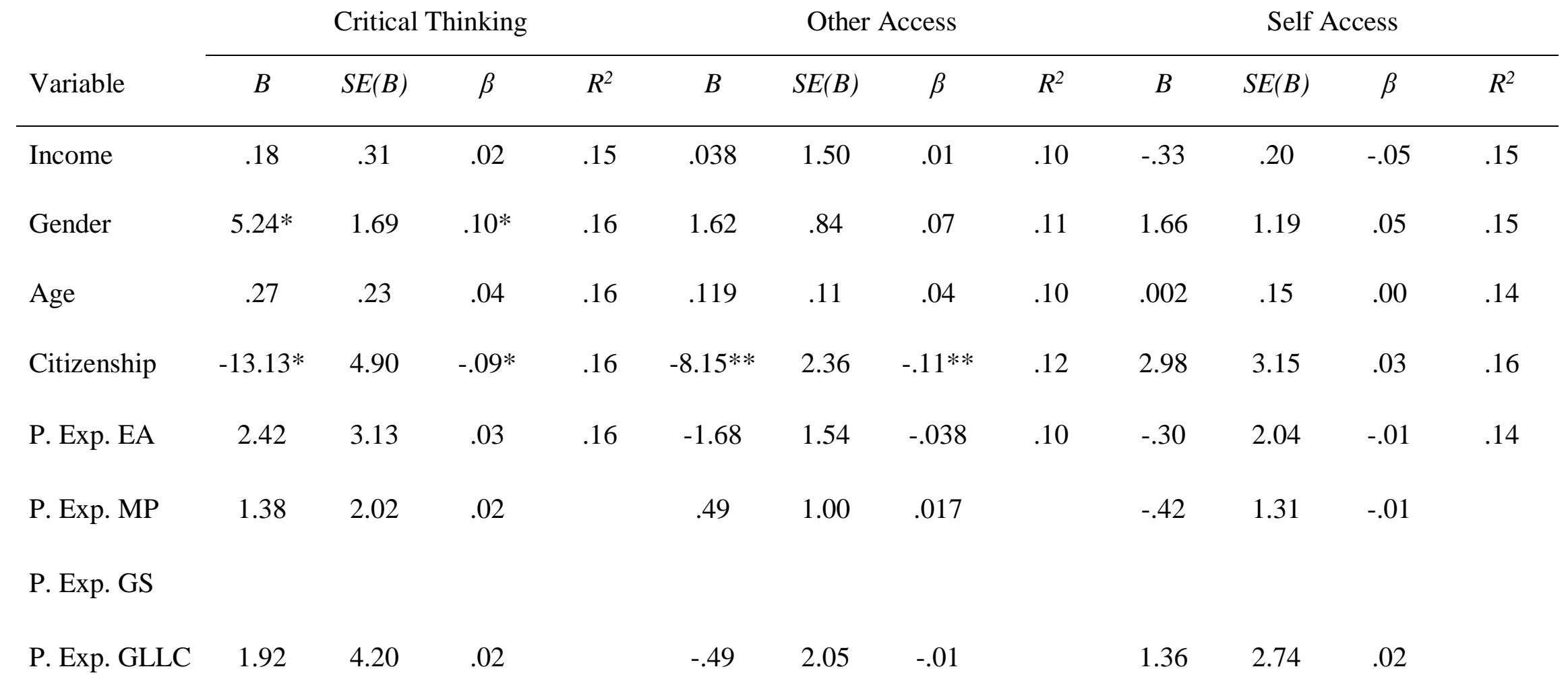

Note: *sig. $p<\alpha=.05$, correcting for multiple comparisons under Benjamini \& Hochberg **sig. $p<0.05 / 42$.

P. Exp. $=$ Prior Experience, EA = Education Abroad, MP = Multicultural Programming, GS = Global Studies coursework, GLLC = Global LivingLearning Community 


\section{Limitations}

As with many co-curricular, experiential practices, GDL practices are rarely mandatory. It is difficult then to create experimental design conditions that would afford generalizability. One of the limitations of this study is the lack of random selection in the dataset. The nature (cost, time, degree requirements) of the experiences means that participants generally self-select into these GDL practices. It is, therefore, not possible to generalize findings beyond this dataset. And, while there are no experimental controls in this study, the research interest is in understanding the differences between practice types not the difference between students who participated and those who did not.

The BEVI measures limit the study in some ways as well. The level of granularity on student characteristics is limited by how the items were coded and the wide array of participants who have taken the BEVI. Race, for example, is captured in nine categories based upon US social constructions of norms that would not have been meaningful to international students taking the measure. Therefore, this important student characteristic was not suitable to include in the analysis. Additionally, duration could not be captured in this study because over half of the sample population did not respond to this item on the BEVI. Alternative options were considered, for example, taking the pre- and post-experience BEVI administration date stamps to calculate the number of days. This approach was unsuitable because individual protocols used to collect the data were not the same. Some institutions collected the BEVI data immediately before and after the GDL experience while others gathered data before but waited until six months post experience to collect Time 2 data. 
In addition to the data-coding limitations, the interpretation of the analysis must consider all associations as the design does not permit causal inference. While there are more sophisticated techniques that would provide stronger causal inference from the data, the research questions are limited to associations between variables. Should any associations be detected, further study would need to be conducted to determine possible mechanisms for causality.

\section{Discussion}

While significant results emerged from the data on three student traits (income, gender, and citizenship), the results in citizenship must be consumed in the context of significant imbalance in the sizes of the two subpopulations, and further analysis is recommended under a more balanced selection.

Finding 1. The inverse relationship between income and Other Awareness gains for Education Abroad participants, while statistically significant, is also of relatively small effect size. In isolation, this finding warrants further attention in a separate study. Wandschneider et al (2015) found complementary results in family income. They found family income had a negative relationship with desire to participate in global learning experiences. The higher the family income, the lower the participant's interest in global learning. This may speak to the negative relationship discovered in this study. For students with higher income, it is more likely that their family has the financial means to travel or provide a range of learning experiences outside of the formal educational endeavor. Therefore, participation in Education Abroad may not be motivated by a desire to experience difference or engage with others for students from higher income 
families. It is possible that career interests and credentialing from foreign institutions is more motivating for the higher income group.

Finding 2. The differences in Critical Thinking and Other Awareness outcomes for Global Studies participants should be approached with caution. The number of US citizens was appreciably larger than non-US citizens and may have some impact on these results. Again, effect size was also fairly small. Further investigation is warranted on this finding.

Finding 3. Prior participation did not predict differences between GDL practice types. This is somewhat in line with findings from the Georgetown Consortium report (Paige, Connor, \& VandeBurg, 2009) where prior participation in study abroad was not correlated with higher Time 1 competencies in self-awareness and other-awareness as measured by an intercultural competence instrument. Further investigation into specific practices could illuminate why these data did not predict differences.

Finding 4. The results indicating the power of gender on Education Abroad outcomes, on the other hand, are present across all outcomes, with statistical significance, and of moderate effect size. Additionally, gender was also explanatory of differential outcomes in Critical Thinking in Global Studies. These results are interesting but not new in the context of the modicum of related literature on the topic.

The impact of differential demographics on education abroad, in particular, is predicted in prior studies. Socio-economic status, major, and language ability are variables that have been explored and found predictive of outcome gains related to awareness of self and others or critical thinking (Basow \& Gaugler, 2017; Connor, Paige \& VandeBerg, 2009; Sutton \& Rubin, 2004; Wandschneider et al, 2015). 
Specifically, the role of gender in outcomes similar to those in this study is treated in the Georgetown Consortium Report (Connor, Paige \& VandBerg 2009) where they suggested that participants in Education Abroad practices who identify as female not only start their experiences with higher self-awareness and other-awareness (as measured through intercultural awareness scales), but they gain significantly more on these outcomes than males during their experiences abroad. Cohen et al (2005) also found that study abroad students who identified as female had scores on intercultural development scales that suggested they identified more with the host culture than did males.

Additionally, Geising (2017) found similar patterns in females participating in short-term Education Abroad practices in the US. Again, females began their experiences at higher capacities of self-awareness and other-awareness, however, in Geising's study, female participants did not gain more than males over the course of the experience. Implications of this research follow in the next section.

In the present study, the greater positive change on the part of females participating in Education Abroad are across all outcomes and significant. Additionally, the Critical Thinking gains seen for females in Global Studies signals a pattern that participants who choose to participate in these two GDL practices are changing their capacity to think critically about self and others more than males. This begs two questions (a) what might be the mechanism(s) behind this difference, and (b) what are the possible implications for program implementation?

\section{Mechanisms Underlying Gender Difference}

Prior research discussed in this article observed differences among males and females participating in GDL practices that complements or contextualizes findings from 
this study. The mechanisms for such differences are worth exploring further in future research. Further exploration could be done to identify potential mechanisms for gender differences in (a) openness to experiencing difference, (b) emotional capacities, (c) identity fixedness, and (d) perceptions of the relevance of global learning to career outcomes.

What might allow or motivate females to be more open to differences they experience during GDL practices? Does that openness result in the growth in critical thinking and awareness capacities observed in studies? Wandschneider and colleagues (2015) demonstrated that female students expressed more interest in global learning experiences. Cohen and colleagues (2005) identified how female participants in education abroad were more likely than males to identify with the host culture and explore its differences from their home culture. Meshkat and Nejati (2017) found that females in their studies tended to express more empathy, emotional self-awareness, and social responsibility than their male counterparts. These studies point to greater capacities for females, in general, to receive, analyze, and make sense of the differences between self and others that they encounter in their GDL experiences.

It would be simplistic, however, to allow this research to point educators to a biological narrative for these outcome differences. Social norms and programmatic variables could also play a large role in GDL practice outcomes. In her study, Giesing (2017) noted how female participants are more emotionally activated due to the context of their GDL practice. She notes that female participants began their experiences with a less fixed notion of who they were and more desire to explore who they are. This 
openness or social freedom to explore new ways of seeing the world and ways of being could be what precipitates disparate gains between genders.

When there is less social pressure to focus on career skills and credentials perhaps students are able to free up cognitive space for thinking about identity and contextualizing identity in the world outside of self. For males, in general, there is considerable pressure to pursue a career. Traditionally, this has meant learning skills and content around a particular subject matter that resulted in a credential desired by employers. With changes in the workforce that favor being able to think critically about self and others, universities may need to reflect upon how they communicate with students of different genders.

\section{Implications for Program Implementation}

Focusing on the findings regarding the influence of gender on Critical Thinking, Self Access, and Other Access scores there are a few implications for scholars and practitioners to bear in mind. First, there is a need to better understand mechanisms and motivations. What variables in the learner or the learning environment favor females in the development of critical thinking and awareness of self and others? What ideas do students have about the importance of these outcomes for their future and from where do they come? Second, educators should be aware of the gender norms that are perceived through the materials they use to communicate about GDL practices. For example, images used in promotional materials of education abroad experiences may steer male students away from participation. Practitioners who design GDL experiences could get ahead of this by developing messages and materials that help students understand the 
changing demands of employers so that male students, in particular, can see how GDL experiences are relevant to their career path. 


\section{References}

Abbe, A., Herman, J., \& Gulik, L. (2007). Cross-cultural competence in army leaders: A conceptual and empirical foundation (Unclassified Report No. 2008-01) (p. 59). Arlington, VA: U.S. Army Research Institute.

Acheson, K., Dirkx, J. M., Ullom, C., Kapadia, S., Bhuyan, D., \& Wiley, J. (In press). Assessing transformation of the self in high impact learning: Methodological considerations. In Cultivating the globally sustainable self: How the human species might fulfill its potential. Oxford, UK: Oxford University Press.

Astin, A. W. (1993). What matters in college? Four critical years revisited. San Francisco, CA: Jossey-Bass.

Banks, C. A. M., \& Banks, J. A. (1995). Equity pedagogy: an essential component of multicultural education. Theory into Practice, 34, 152-158.

Basow, S. A., \& Gaugler, T. (2017). Predicting adjustment of U.S. college students studying abroad: Beyond the multicultural personality. International Journal of Intercultural Relations, 56, 39-51.

Benjamini, Y., \& Hochberg, Y. (1995). Controlling the false discovery rate: A practical and powerful approach to multiple testing. Journal of the Royal Statistical Society Series B (Methodological), (57), 289-300.

Bennett, M. (1998). Intercultural communication: A current perspective. In Basic Concepts of Intercultural Communication (1st ed., pp. 1-34). Yarmouth, ME: Intercultural Press. 
Braskamp, L. A., Braskamp, D. C., \& Merrill, K. (2009). Assessing Progress in Global Learning and Development of Students with Education Abroad Experiences. Frontiers: The Interdisciplinary Journal of Study Abroad, 18, 101-118.

Brownell, J., \& Swaner, L. (2010). Five high-impact practices: Research on learning outcomes, completion and quality. Washington, D.C.: Association of American Colleges and Universities.

Cohen, A., Paige, M., Shively, R., Emert, H., \& Hoff, J. (2005). Maximizing study abroad through language and culture strategies: Research on students, study abroad program professionals, and language instructors (pp. 1-414). Minneapolis, MN: Center for Advanced Research on Language Acquisition at the University of Minnesota.

Connell, C. (2016). The Ascent of Global Learning. International Educator, 25(2), 1623.

Connor-Linton, J., Paige, R. M., \& Vande Berg, M. (2009). The Georgetown consortium project: Interventions for student learning abroad. Frontiers Journal, 18(Fall 2009), 1-75.

Dabrowski, K. (1964). Positive disintegration. Boston, MA: Little Brown.

Deardorff, D. (Ed.). (2009). The Sage handbook of intercultural competence. Thousand Oaks, CA: Sage Publications.

DeTurk, S. (2006). The power of dialogue: Consequences of intergroup dialogue and their implications for agency and alliance building. Communication Quarterly, $54(1), 33-51$. 
Dirkx, J. M. (1998). Transformative learning theory in the practice of adult education: An overview. PAACE Journal of Lifelong Learning, 7, 1-14.

Dweck, C. S. (2008). Mindset: the new psychology of success. New York, NY: Ballantine Books.

Engle, L., \& Engle, J. (2003). Study abroad levels: Toward a classification of program types. Frontiers Journal, 9(Fall 2003), 1-20.

Friedman, J., Haverkate, V., Oomen, B., Park, E., \& Sklad, M. (2015). Going glocal in higher education: The theory, teaching and measurement of global citizenship. Middlebury, the Netherlands: de Drvkkery.

Galinsky, A., \& Maddux, W. (2009). Cultural borders and mental barriers: The relationship between living abroad and creativity. Journal of Personality and Social Psychology, 96(5), 1047-1061.

Gardner, P., Gross, L., \& Steglitz, I. (2008). Unpacking your study abroad experience: Critical reflection for workplace competencies (Brief No. 1). Michigan State: Michigan State University.

Giesing, W. (2017). MEPI, BEVI, and EI leadership: Implications and applications for global leadership assessment and development. (Dissertation) James Madison University, Harrisonburg, VA.

Green, M. (2013). Improving and assessing global learning. Washington, D.C.: NAFSA: Association of International Educators.

Grunzweig, W., \& Reinhart, N. (2002). Rockin' in Red Square: Critical approaches to international education in the age of cyberculture. Munster, Germany: Lit Verlag. 
Hart Research Associates. (2016). Recent trends in general education design, learning outcomes, and teaching approaches (Key findings from a survey among administrators at AAC\&U member institutions). Washington, DC: Association of American Colleges \& Universities.

Hammer, M. (2009). Solving Problems and Resolving Conflict Using the Intercultural Conflict Style Model and Inventory. In M. Moodian (Ed.), Contemporary Leadership and Intercultural Competence: Exploring the Cross-Cultural Dynamics within Organizations (pp. 219-232). Thousand Oaks, CA: Sage Publications.

Hayes, D. J. (2001). A comparison of evangelical Christians and mental health professionals on the Beliefs, Events, and Values Inventory (BEVI) (Unpublished dissertation). James Madison University. Retrieved from Dissertation Abstracts International.

Hoggan, C. D. (2016). Transformative learning as a metatheory. Adult Education Quarterly, 66(1), 57-75.

Hovland, K. (2014). Global learning: Defining, designing, demonstrating. Washington, D.C.: Joint publication of AAC\&U and NAFSA: Association of International Educators.

Iyer, C. (2013). Assessing and Engaging Beliefs and Values in a Learning Community of U.S. and International Students: Implications and Applications from the Forum BEVI Project (Unpublished dissertation). James Madison University, Harrisonburg, VA. 
Kahn, H. E., \& Agnew, M. (2017). Global learning through difference: Considerations for teaching, learning, and the internationalization of higher education. Journal of Studies in International Education, 21(1), 52-64.

Kilgo, C. (2016). An epistemological revolution: Using quantitative data to critically interrogate high-impact educational practices (Unpublished dissertation). The University of Iowa.

Kilgo, C., Ezell-Sheets, J., \& Pascarella, E. (2015). The link between high-impact practices and student learning: some longitudinal evidence. Higher Education (00181560), 69(4), 509-525.

Kolb, D. (2015). Experiential learning: Experience as the source of learning and development (Second). Upper Saddle River, NJ: Pearson Education, Inc.

Kuh, G. (2005). Assessing conditions to enhance educational effectiveness: The inventory for student engagement and success (1st ed.). San Francisco, CA: Jossey-Bass.

Kuh, G. (2008). High-Impact educational practices: What they are, who has access to them, and why they matter. Washington, D.C.: American Association of Colleges and Universities.

Kuh, G., \& O’Donnell, K. (2013). Ensuring quality \& taking high-impact practices to scale. Washington, D.C.: American Association of Colleges and Universities.

Law, A. (2014). Education abroad's evolving role in higher educaiton. Impact of the Arcadia Experience Abroad, (2014), 12-15.

Lou, K., Paige, R., \& Vande Berg, M. (2012). Student learning abroad: What our students are learning, what they're not, and what we can do about it. Sterling, VA: Stylus Publishing. 
Maki, P., \& Schneider, C. G. (2015). Assessment that works: A national call, a twentyfirst century response. Washington, D.C.: Association of American Colleges and Universities.

Medina-Lopez-Portillo, A. (2004). Intercultural learning assessment: The link between program duration and the development of intercultural sensitivity. Frontiers Journal of International Education, 10(Fall), 179-199.

Meshkat, M., \& Nejati, R. (2017). Does Emotional Intelligence Depend on Gender? A Study on Undergraduate English Majors of Three Iranian Universities. Sage Open, 7(3).

Mezirow, J. (1997). Transformative learning: theory to practice. New Directions for Adult and Continuing Education, 74(Summer), 5-12.

Ogden, A. (2006). Ethnographic inquiry: Reframing the learning core of education abroad. Frontiers: The Interdisciplinary Journal of Study Abroad, XIII(Fall), 87112.

Ogden, A. (2015). Toward a research agenda for U.S. education abroad. Washington, D.C.: Association of International Education Administrators.

Open Doors Data. (2016). Opens Doors Annual Report [Institute of International Education]. Retrieved from http://iie.org/Research-and-Publications/OpenDoors/Data\#.WBozfOMrKUk

Piaget, J. (1952). The origins of intelligence in children; New York, NY: International Universities Press. 
Pike, G., Kuh, G., \& McCormick, A. (2011). An investigation of the contingent relationships between learning community participation and student engagement. Research in Higher Education, 52(3), 300-322.

Provencher, A., \& Kassel, R. (2017). High-impact practices and sophomore retention: Examining the effects of selection bias. Journal of College Student Retention: Research, Theory \& Practice, 18(3), 1-21.

Reisweber, J. R. (2008). Beliefs, values, and the development of intercultural awareness (Unpublished dissertation). James Madison University, Harrisonburg, VA.

Salisbury, M. (2015). Matching program and student characteristics with learning outcomes. In N. Sobania (Ed.), Putting the local in global education (pp. 36-51). Sterling, VA: Stylus Publishing.

Sandeen, C. (2012). High-Impact educational practices: What we can learn from the traditional undergraduate setting. Continuing Higher Education Review, 76.

Shealy, C. N. (2004). A model and method for "making" a combined-integrated psychologist: Equilintegration (EI) theory and the Beliefs, Events, and Values Inventory (BEVI). Journal of Clinical Psychology, 60(10), 1065-1090.

Shealy, C. N. (2006). The Beliefs, Events, and Values Inventory (BEVI): Overview, implications, and guidelines. Harrisonburg, VA.

Shealy, C. N. (Ed.). (2015). Making sense of beliefs and values: Theory, research, practice (1st ed.). New York, NY: Springer Publishing. 
Stebleton, M., Soria, K., \& Cherney, B. (2013). The high impact of education abroad: College students' engagement in international experiences and the development of intercultural competencies. Frontiers Journal, 22, 1-24.

Sutton, R. C., \& Rubin, D. L. (2004). The GLOSSARI project: Initial findings from a system-wide research initiative on study abroad learning outcomes. Frontiers: The Interdisciplinary Journal of Study Abroad, 10, 65-82.

Vygotsky, L. (1997). Educational psychology. (R. Silverman, Trans.). Boca Raton, FL: St. Lucie Press.

Wandschneider, E., Pysarchik, D. T., Sternberger, L. G., \& Ma, W. (2015). The Forum BEVI project: Applications and implications for international, multicultural, and transformative learning. Frontiers: The Interdisciplinary Journal of Study Abroad, 25(Spring), 150-228.

Whitehead, D. M. (2015). Global learning: Key to making excellence inclusive. Liberal Education, (Summer), 6-13.

Wilson-Mulnix, J. (2012). Thinking critically about critical thinking. Educational Philosophy and Theory, 44(5), 464-479. 


\section{SECTION SIX}

SCHOLARLY REFLECTION 


\section{Scholarly Reflection}

"To study and not think is a waste. To think and not study is dangerous." (Confucius, 1938/500, v 2:15)

As I began this 'think piece' I found myself having been trained to look for evidence of change or 'influence' in my practice and scholarship. I was looking for something to study as I thought about whether I had changed. Figure 1 represents one way I found I could look to for some evidence of change. When Cohort 10 began its first summer of residential instruction, working groups were organized, in part, through data that came from the StrengthsFinder survey (Rath, 2007). The instrument is designed to identify areas where individuals can develop their talents in interpersonal and intrapersonal interactions. My results were somewhat consistent with the way I saw myself, but I did not think about the report beyond the summer term.

In February of this year, I was invited to join a group of scholars to start a new consulting group. As part of the group building process, we decided to take the StrengthsFinder (Rath, 2007) because one of the group members had used this instrument in his professional role to help teams troubleshoot intragroup challenges. Though I cannot isolate the change I see here to the dissertation process, the data still appears to be a useful bookend to my program and framework for this reflection. The skills and dispositions I have acquired during my coursework would arguably culminate in this dissertation. As I respond to the reflection prompts, I will refer back to this data as I make meaning of this potential evidence of change. 


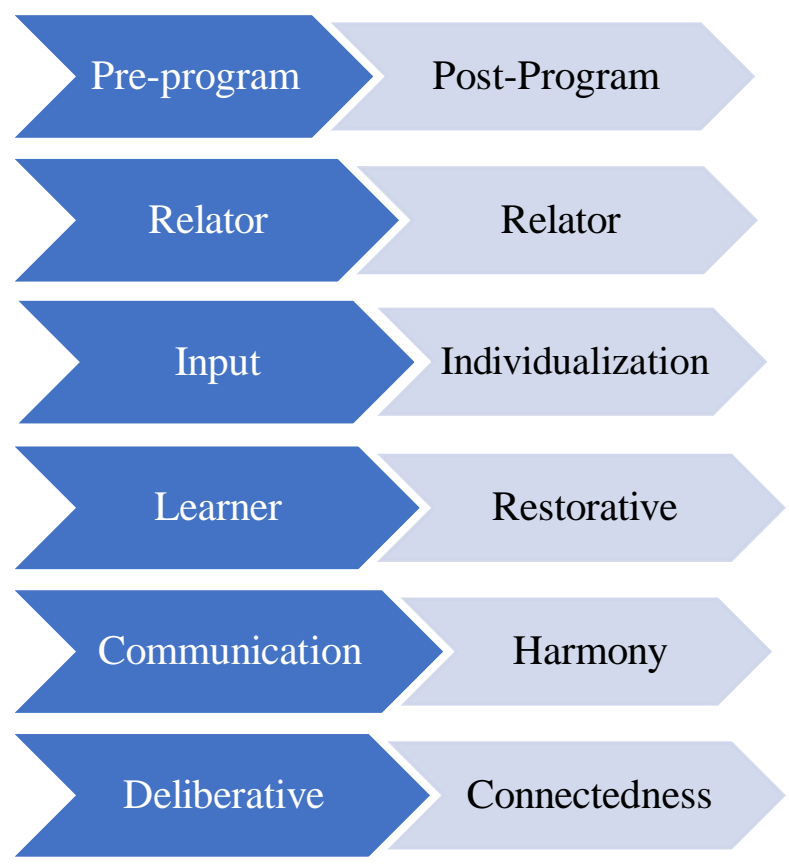

Figure 4. StrengthsFinder (Rath, 2007) top five traits pre-program and post-program

\section{How has the dissertation influenced your practice as an educational leader?}

In February of this year, I attended my first conference of International Education administrators. At this meeting in Washington, DC, senior international officers from universities around the world came together for their own annual moment of discussion and reflection. The theme of the conference was Senior International Officers as Change Agents. This was my moment to try on how it might feel to have a formal leadership role at an institution. I was excited to be in the middle of my dissertation process, having read work by some of the leaders present at the conference and to get the opportunity to discuss what I had learned thus far. That experience led me to an acute awareness of the cultural suicide that global and diversity learning practices could precipitate in the students I study.

If I have your attention, I will set the context by offering a baseline for my leadership in my field then I will explain what I mean. When I began my doctoral 
program, I was in my second full year of managing a consulting partnership that served higher education through supporting international offices in program evaluation and assessment, intercultural awareness training, and curriculum design. My partners and I had landed a multi-year, multi-site large scale program evaluation for a university provider of education abroad experiences. This experience propelled me into a position of leadership as my partners and I helped this institution understand the lived experiences of their students abroad and the influence of their programs on the host communities where their students lived.

Projecting forward two years, from that successful evaluation experience and readings for my dissertation, I have learned just how much institutions focus on performance indicators and indirect measures of change like participation numbers instead of more direct measures of desired outcomes like those in my dissertation or what I saw during my evaluation work. The handful of scholars with whom I spoke all agreed on this point, but I had not entirely considered how most of the remaining seven hundred participants in the room were the embodiment of 'institution' in my prior statement. These were the people who were responsible for the head counting behaviors--whether through inherited practice or lack of knowledge of best practice. During a conversation with a senior international officer from a well-endowed private university in the northeastern US, I realized this point and all the implications it would have for my choice to join this group as a colleague. He engaged me in conversation about my dissertation and after listing a few preliminary findings, he said, "You know, I never really thought about how a student's background might influence his learning abroad, huh.” My immediate thought was not a productive one, but then I realized this was an opportunity 
for me to employ a strength, and with luck, not find myself ostracized from the herd or committing cultural suicide because of my beliefs about why students should participate in global and diversity learning practices.

In a moment where I might have more easily walked away and relegated this administrator to the clueless pile, I drew upon my strength. Relator is the only StrengthsFinder (Rath, 2007) trait that I have maintained across the three years of my program. I still find it important to dig deeply into my relationships with colleagues and students, take risks by being vulnerable in those interactions, and seek to understand what is important to people and why. Through holding onto the moment and being authentic, this administrator heard the voices of students with whom I had connected abroad. He offered me a job at the end of the conversation.

The knowledge that I gain through my dissertation process, when just studied, could be a waste when left on paper and not applied, at best. At worst, the knowledge that I have gained through this process could be a point of differentiation that leads me to being excluded if I wield it like a badge of courage. However, through seeking to relate to people, I find that I can use the knowledge I gained to lead people to better practice. I choose to see this playing out in my StrengthsFinder (Rath, 2007) changes where I moved from a heavy focus on data and knowledge as valuable by themselves to Individualization, which StrengthsFinder defines as being focused on the uniqueness in each colleague I meet. This shift would suggest that I seek to explore ways for people who are different from one another to work well together. In the current era of political polarization, it seems like that should be a job requirement for every educational leader. 
Doing the research for my dissertation has afforded me insight that administrators need to understand the practices over which they hold sway. My findings relate how impactful global and diversity learning practice can be, both positive and negative. It is important for leaders to understand exactly what they are asking students to do by changing their awareness and becoming a more critical thinker--for some it will be cultural suicide from their more traditional, monocultural communities. We talk about it casually, and, as practitioners, grow impatient with students who resist change, but we overlook the fundamental needs that are met by the worldviews with which students enter our classrooms. Through my scholarship and the translation of heady scholarship that is essential for understanding what happens inside global and diversity learning practices, I can lead others to this awareness.

In April, I was one of two panelists who delivered a webinar on assessing global learning hosted by the largest international education network in the US. There were several hundred participants logged into the call asking me for advice about how to approach their own practices. This may be the more conventional look to leadership, but it does not feel as meaningful as the long-term relationships I build through taking a walk with a colleague and having her pick my brain about what I have seen and asking her questions about why global and diversity outcomes are important.

\section{How has the dissertation process influenced you as a scholar?}

Reason and emotion do not exist separate from each other, despite our efforts in education to make it so (Taylor \& Cranton, 2012). This thought that has been reinforced by my dissertation research is likely the most influential on my scholarship. So much of what we think of as scholarship is removed from the emotion of lived experiences in the 
name of being objective. I think academia has lost balance on this point and is losing the good will of the populous in some part to that. My own trajectory in StrengthsFinder (Rath, 2007) is notable on this point. It highlights how I thought about scholarship prior to this process.

Entering the process, I was a Learner. I wanted knowledge for the sake of being knowledgeable. I wanted to control the facts and master understandings of difficult concepts. As I look at the difficult work that students do to figure out who they are as a result of global and diversity learning experiences, I realize how vain and misplaced that energy was. My new orientation toward Restorative signals to me a shift in looking at knowledge for myself to searching for knowledge for others that can restore relationships and solve problems.

Upon entry, my StrengthsFinder (Rath, 2007) results would have predicted scholarship, writing and argument to be something that came more easily to me. While I do not think I have lost those scholarly tools, I have found a more nuanced use for them. Harmony is what I traded for Communication. Again, this is a shift away from having a spotlight on me where information is coming from the fountain of wisdom. I would prefer as a scholar to be the person with a will to bring voices together and project voices of others who cannot be heard on their own.

Finally, I entered the Ivory Tower with the caution that comes with have a Deliberative strength. In my scholarly writing, I wanted to be serious, careful, and private or removed from emotion. Though thinking about how I would project my writing, I have shifted away from the private, careful, serious scholar to the scholar who can bring connections out into the open. Practitioners in need see the connections 
between their assumptions of how students gain awareness, who the students are, and the learning environments they design. It is my hope that my research will be a small step toward that outcome.

As I conclude this process of reflection I can say that I have identified points of influence on my leadership and scholarship as a result of my dissertation process. I have become more outward facing and desirous of connection. To be sure, some of that has to do with the isolation that comes from reading and writing, but I also feel the responsibility of a leader to project the voices of students through my scholarship. 


\section{REFERENCES}

Abbe, A., Herman, J., \& Gulik, L. (2007). Cross-cultural competence in army leaders: A conceptual and empirical foundation (Unclassified Report No. 2008-01; p. 59). Arlington, VA: U.S. Army Research Institute.

Acheson, K., Dirkx, J. M., Ullom, C., Kapadia, S., Bhuyan, D., \& Wiley, J. (In press). Assessing transformation of the self in high impact learning: Methodological considerations. In C. Shealy (Ed), Cultivating the globally sustainable self: How the human species might fulfill its potential. Oxford, UK: Oxford University Press.

Agnew, M. (2012). Strategic planning: An examination of the role of disciplines in sustaining internationalization of the university. Journal of Studies in International Education, 17(2), 183-202.

Agnew, M., \& Kahn, H. E. (2014). Internationalization-at-home: Grounded practices to promote intercultural, international, and global learning. Metropolitan Universities, 25(3), 31-46.

Agnew, M., \& VanBalkom, W. D. (2009). Internationalization of the university: Factors impacting cultural readiness for organizational change. Intercultural Education, $20(5), 451-462$.

Allport, G. (1954). The nature of prejudice. New York, NY: Double Day.

Altbach, P., Gumport, P., \& Berdahl, R. (2011). American higher education in the twenty-first century: Social, political, and economic challenges (3rd ed.). Baltimore, MD: John Hopkins University Press. 
Association of American Colleges \& Universities. (2011). The LEAP vision for learning: Outcomes, impact, and employer's views (Liberal Education and America's Promise). Washington, D.C.

Astin, A. W. (1993). What matters in college? Four critical years revisited. San Francisco, CA: Jossey-Bass.

Banks, C. A. M., \& Banks, J. A. (1995). Equity pedagogy: An essential component of multicultural education. Theory into Practice, 34, 152-158.

Basow, S. A., \& Gaugler, T. (2017). Predicting adjustment of U.S. college students studying abroad: Beyond the multicultural personality. International Journal of Intercultural Relations, 56, 39-51.

Benjamini, Y., \& Hochberg, Y. (1995). Controlling the false discovery rate: A practical and powerful approach to multiple testing. Journal of the Royal Statistical Society Series B (Methodological), (1:57), 289-300.

Bennett, J. M., \& Bennett, M. J. (1994). Multiculturalism and international education: Domestic and international differences. In G. Althen (Ed.), Learning across cultures (pp. 145-165). Washington, D.C.: National Association of International Educators.

Bennett, M. (1998). Intercultural communication: A current perspective. In M. Bennet (Ed), Basic Concepts of Intercultural Communication (pp. 1-34). Yarmouth, ME: Intercultural Press.

Birnbaum, R. (1989). The cybernetic institution: Toward an integration of governance theories. Higher Education, 18(2), 239-253. 
Birnbaum, R. (2004). The end of shared governance: Looking ahead or looking back. New Directions for Higher Education, 2004(127), 5-22. https://doi.org/10.1002/he.152

Bolden, R., Gosling, J., \& O’Brien, A. (2012). Academic leadership: Changing conceptions, identities and experiences in UK higher education. Presented at the Leadership Foundation for Higher Education and Exeter University, London, UK.

Bolen, M. C. (2007). A guide to outcomes assessment in education abroad. Carlisle, PA: Forum on Education Abroad.

Bolman, L. G., \& Deal, T. E. (2013). Reframing organizations: Artistry, choice \& leadership (5th ed.). San Fransisco, CA: Jossey-Bass.

Braskamp, L. A., Braskamp, D. C., \& Merrill, K. (2009). Assessing progress in global learning and development of students with education abroad experiences. Frontiers: The Interdisciplinary Journal of Study Abroad, 18, 101-118.

Braskamp, L. A., \& Engberg, M. E. (2011). How colleges can influence the development of a global perspective. Liberal Education, 97(3/4), 34-39.

Brewster, L., Dyjak-LeBlanc, K., Grande, S., White, R., \& Shullman, S. (2016). The EI leadership model: From theory and research to real world application. In C. N. Shealy (Ed.), Making Sense of Beliefs and Values (pp. 530-574). New York, NY: Springer Publishing.

British Council. (2013). Culture at work: The value of intercultural skills in the workplace (Higher Education). London, UK: British Councils.

Brower, A. M., \& Inkelas, K. K. (2010). Living-learning programs. Liberal Education, 96(2), 36-43. 
Brownell, J., \& Swaner, L. (2010). Five high-impact practices: Research on learning outcomes, completion and quality. Washington, D.C.: Association of American Colleges and Universities.

Bu, L. (1999). Educational exchange and cultural diplomacy in the cold war. Journal of American Studies, 33(03), 393-415.

Cohen, A., Paige, M., Shively, R., Emert, H., \& Hoff, J. (2005). Maximizing study abroad through language and culture strategies: Research on students, study abroad program professionals, and language instructors. Minneapolis, MN: Center for Advanced Research on Language Acquisition at the University of Minnesota.

Confucious. (1938). The analects of Confucious. (A. Waley, Trans.) (18th ed.). New York, NY: Random House.

Connell, C. (2016). The ascent of global learning. International Educator, 25(2), 16-23.

Connor-Linton, J., Paige, R. M., \& Vande Berg, M. (2009). The Georgetown consortium project: Interventions for student learning abroad. Frontiers Journal, 18(Fall 2009), 1-75.

Cornwell, G. H., \& Stoddard, E. W. (1999). Globalizing Knowledge: Connecting International \& Intercultural Studies. The Academy in Transition. Washington, D.C.: Association of American Colleges and Universities.

Creswell, J. (2012). Educational research: Planning, conducting, and evaluating quantitative and qualitative research (4th ed.). New York, NY: Pearson.

Dabrowski, K. (1964). Positive disintegration. Boston, MA: Little Brown. 
Deardorff, D. (Ed.). (2009). The Sage handbook of intercultural competence. Thousand Oaks, CA: Sage Publications, Inc.

Del Favero, M., \& Bray, N. (2005). The faculty-administrator relationship: Partners in prospective governance? Scholar-Practitioner Quarterly, 3(1), 53-72.

Dessel, A., \& Rogge, M. (2008). Evaluation of intergroup dialogue: A review of empirical literature. Conflict Resolution Quarterly, 26(2), 199-238.

DeTurk, S. (2006). The power of dialogue: Consequences of intergroup dialogue and their implications for agency and alliance building. Communication Quarterly, 54(1), 33-51.

Dirkx, J. M. (1998). Transformative learning theory in the practice of adult education: An overview. PAACE Journal of Lifelong Learning, 7, 1-14.

Dirkx, J. M. (2012). Self-formation and transformative learning: A response to "Calling transformative learning into question: Some mutinous thoughts," by Michael Newman. Adult Education Quarterly, 62(4), 399-405.

Dweck, C. S. (2008). Mindset: The new psychology of success. New York, NY: Ballantine Books.

Dwyer, M. (2004). More is better: The impact of study abroad program duration. Frontiers: The Interdisciplinary Journal of Study Abroad, 10(Fall), 151-163.

Engle, L., \& Engle, J. (2003). Study abroad levels: Toward a classification of program types. Frontiers Journal, 9(Fall 2003), 1-20.

Engle, L., \& Engle, J. (2004). Assessing language acquisition and intercultural sensitivity development in relation to study abroad program design. Frontiers: The Interdisciplinary Journal of Study Abroad, 10, 219-236. 
Ettling, D. (2012). Educator as change agent: Ethics of transformative learning. In E. W. Taylor \& P. Cranton (Eds.), The handbook of transformative learning (pp. 536551). San Francisco, CA: Jossey-Bass.

Feather, N. T. (1992). Values, valences, expectations, and actions. Journal of Social Sciences, 48(2), 109-152.

Friedman, J., Haverkate, V., Oomen, B., Park, E., \& Sklad, M. (2015). Going glocal in higher education: The theory, teaching and measurement of global citizenship. Middlebury, the Netherlands: de Drvkkery.

Galinsky, A., \& Maddux, W. (2009). Cultural borders and mental barriers: The relationship between living abroad and creativity. Journal of Personality and Social Psychology, 96(5), 1047-1061.

Gardner, P., Gross, L., \& Steglitz, I. (2008). Unpacking your study abroad experience: Critical reflection for workplace competencies (Brief No. 1). Ann Arbor: Michigan State University.

George, B., Sims, P., McClean, A., \& Mayer, D. (2011). Discovering your authentic leadership. In On Leadership. Boston, MA: Harvard Business School Publishing Corporation.

Giesing, W. (2017). MEPI, BEVI, and EI leadership: Implications and applications for global leadership assessment and development. (Unpublished dissertation) James Madison University, Harrisonburg, VA.

Goleman, D. (2011). What makes a leader? In On Leadership (pp. 1-21). Boston, MA: Harvard Business School Publishing Corporation. 
Goode, M. (2008). The role of faculty study abroad directors: A case study. Frontiers: The Interdisciplinary Journal of Study Abroad, 25(Winter), 149-172.

Gorski, P. C. (2008). Good intentions are not enough: A decolonizing intercultural education. Intercultural Education, 19(6), 515-525.

Green, M. (2013). Improving and assessing global learning. Washington, D.C.: NAFSA: Association of International Educators.

Grunzweig, W., \& Reinhart, N. (2002). Rockin' in Red Square: Critical approaches to international education in the age of cyberculture. Munster, Germany: Lit Verlag.

Gurin, P., Dey, E., Hurtado, S., \& Gurin, G. (2002a). Diversity and higher education: Theory and impact on educational outcomes. Harvard Educational Review, 72(3), $330-367$.

Hammer, Mitchell R., Bennett, M. J., \& Wiseman, R. (2003). Measuring intercultural sensitivity: The intercultural development inventory. International Journal of Intercultural Relations, 27(4), 421-443.

Hammer, M. (2009). Solving problems and resolving conflict using the intercultural conflict style model and inventory. In M. Moodian (Ed.), Contemporary leadership and intercultural competence: Exploring the cross-cultural dynamics within organizations (pp. 219-232). Thousand Oaks, CA: Sage Publications. Hart Research Associates. (2008). How should college assess and improve student learning: Employers' views on the accountability challenge (Commissioned Research Report). Washington, D.C.: Association of American Colleges \& Universities. 
Hart Research Associates. (2016). Recent trends in general education design, learning outcomes, and teaching approaches (Key findings from a survey among administrators at AAC\&U member institutions). Washington, DC: Association of American Colleges \& Universities.

Hathaway, J., Pilonieta, P., \& Medina, A. (2015). How preservice teachers'study abroad experiences lead to changes in their perceptions of English language learners. Frontiers Journal, 25(Spring), 73-90.

Hayes, D. J. (2001). A comparison of evangelical Christians and mental health professionals on the Beliefs, Events, and Values Inventory (BEVI) (Unpublished doctoral dissertation). James Madison University, Harrisonburg, VA.

Hoffa, W. (2007). A history of US study abroad: beginnings to 1965. Carlisle, PA: Frontiers.

Hoffa, W., \& DePaul, S. (2010). A history of U.S. Study Abroad: 1965-present. Carlisle, PA: Frontiers.

Hoggan, C. D. (2016). Transformative learning as a metatheory. Adult Education Quarterly, 66(1), 57-75.

Hovland, K. (2014). Global learning: Defining, designing, demonstrating. Washington, D.C.: Joint publication of AAC\&U and NAFSA: Association of International Educators.

Hudzik, J. (2011). Comprehensive internationalization: From concept to action. Washington, D.C.: NAFSA. 
Ibarra, H. (n.d.). Context Diversity: Reframing Higher Education In The 21st Century. Retrieved from https://compact.org/resource-posts/context-diversity-reframinghigher-education-in-the-21st-century/

Indiana University Center for Postsecondary Research. (2007). Experiences that matter: Enhancing student learning and success (National Survey of Student Engagement). Bloomington, IN.

Inkelas, K. K., \& Weisman, J. L. (2003). Different by design: An examination of student outcomes among participants in three types of living-learning programs. Journal of College Student Development, 44(3), 335-368.

Institute of International Education. (2016). Open doors 2016: Report on international education exchange. New York, NY: Institute for International Education.

Intolubbe-Chmil, L., Spreen, C. A., \& Swap, R. (2012). Transformative learning: Participant perspectives on international experiential education. Journal of Research in International Education, 11(2), 165-180.

Iyer, C. (2013). Assessing and Engaging Beliefs and Values in a Learning Community of U.S. and International Students: Implications and Applications from the Forum BEVI Project (Unpublished dissertation). James Madison University, Harrisonburg, VA.

Kahn, H. E., \& Agnew, M. (2017). Global learning through difference: Considerations for teaching, learning, and the internationalization of higher education. Journal of Studies in International Education, 21(1), 52-64.

Kahnemann, D. (2003). A perspective on judgment and choice: Mapping bounded rationality. American Psychologist, 58(9), 697-720. 
Kezar, Carducci, \& Contreras-McGavin. (2006). A World Apart: New Paradigms of Leadership (ASHE Higher Education Report).

Kilgo, C. (2016). An epistemological revolution: Using quantitative data to critically interrogate high-impact educational practices (Unpublished dissertation). University of Iowa, Iowa City, IA.

Kilgo, C., Ezell-Sheets, J., \& Pascarella, E. (2015). The link between high-impact practices and student learning: some longitudinal evidence. Higher Education (00181560), 69(4), 509-525.

Kinzie, J., Kuh, G., Cruce, T., \& Shoup, R. (2008). Unmasking the effects of student engagement on first-year college grades and persistence. The Journal of Higher Education, 79(5), 540-563.

Knight, J., \& Altbach, P. (2016). The internationalization of higher education: Motivations and realities. In P. Altbach (Ed.), Global Perspectives on Higher Education (pp. 105-120). Baltimore, MD: John Hopkins University Press.

Kolb, D. (2015). Experiential learning: Experience as the source of learning and development. Upper Saddle River, NJ: Pearson Education, Inc.

Kuh, G. (2005). Assessing conditions to enhance educational effectiveness : The inventory for student engagement and success (1st ed.). San Francisco, CA: Jossey-Bass.

Kuh, G. (2008). High-Impact educational practices: What they are, who has access to them, and why they matter. Washington, D.C.: American Association of Colleges and Universities. 
Kuh, G., Cruce, T., Shoup, R., \& Kinzie, J. (2008). Unmasking the effects of student engagement on first-year college grades and persistence. The Journal of Higher Education, 79(5), 540-563.

Kuh, G., Pike, G., \& McCormick, A. (2011). An investigation of the contingent relationships between learning community participation and student engagement. Research in Higher Education, 52(3), 300-322.

Kuh., G., Ikenberry, S., Jankowski, N., Reese Cain, T., Ewell, P., Hutchings, P., \& Kinzie, J. (2015). Using evidence of student learning to improve higher education. San Francisco, CA: Jossey-Bass.

Kuh, G., \& O’Donnell, K. (2013). Ensuring quality \& taking high-impact practices to scale. Washington, D.C.: American Association of Colleges and Universities.

Law, A. (2014). Education abroad's evolving role in higher educaiton. Impact of the Arcadia Experience Abroad, (2014), 12-15.

Lee, C. S., Therriault, D. J., \& Linderholm, T. (2012). On the cognitive benefits of cultural experience: Exploring the relationship between studying abroad and creative thinking. Applied Cognitive Psychology, 26(5), 768-778.

Lenning, O. T., \& Ebbers, L. H. (1999). The powerful potential of learning communities: improving education for the future (ASHE Higher Education Report No. 26(6)). Washington, DC: George Washington University.

Lou, K., Paige, R., \& Vande Berg, M. (2012). Student learning abroad: What our students are learning, what they're not, and what we can do about it. Sterling, VA: Stylus Publishing., LLC. 
Maki, P., \& Schneider, C. G. (2015). Assessment that works: A national call, a twentyfirst century response. Washington, D.C.: Association of American Colleges and Universities.

Medina-Lopez-Portillo, A. (2004). Intercultural learning assessment: The link between program duration and the development of intercultural sensitivity. Frontiers Journal of International Education, 10(Fall), 179-199.

Merriam, S. B., \& Bierema, L. L. (2014). Critical thinking and critical perspectives. In Adult Learning Linking Theory and Practice (First, pp. 212-237). San Fransisco, CA: Jossey-Bass.

Meshkat, M., \& Nejati, R. (2017). Does Emotional Intelligence Depend on Gender? A Study on Undergraduate English Majors of Three Iranian Universities. SAGE Open, 7(3).

Mezirow, J. (1997). Transformative learning: Theory to practice. New Directions for Adult and Continuing Education, 74(Summer), 5-12.

Milem, J., Chang, M., \& Antonio, A. (2005). Making diversity work on campus: A research-based perspective (White Paper). Washington, DC: Association of American Colleges and Universities.

Mintzberg, H. (2005). The five basic parts of an organization. In J. Shaftriz, J. Ott, \& Jang, Y. (Eds.), Classics of organization theory (6th ed., pp. 219-230). Belmont, CA: Wadsworth Cengage Learning.

Nagda, B., Gurin, P., Sorenson, N., \& Zúñiga, X. (2009). Evaluating intergroup dialogue: Engaging diversity for personal and social responsibility. Diversity \& Democracy, 12(1), 1-7. 
Ogden, A. (2006). Ethnographic inquiry: Reframing the learning core of education abroad. Frontiers: The Interdisciplinary Journal of Study Abroad, XIII(Fall), 87112.

Ogden, A. (2007). The view from the veranda: Understanding today's colonial student. Frontiers Journal of International Education, 15(Spring), 35-56.

Ogden, A. (2010). Education abroad and the making of global citizens: Assessing learning outcomes of course-embedded, faculty-led international programming (Unpublished dissertation). Pennsylvania State University, Penn Station, PA.

Ogden, A. (2015). Toward a research agenda for U.S. education abroad. Washington, D.C.: Association of International Education Administrators.

Ogden, A., \& Streitwieser, B. (2016). Heralding the scholar-practitioner in international education. In A. Ogden and B. Streitweiser (Eds.). International Education's Scholar-Practitioners: Bridging research and practice (pp. 19-38). Oxford, UK: Symposium Books.

Olson, K., Evans, R., \& Shoenberg, R. (2007). At home in the world: Bridging the gap between Internationalization and multicultural education (Global Learning for All). Washington, D.C.: American Council on Education.

Olson, K., Green, M., \& Hill, B. (2006). A handbook for advancing comprehensive internationalization: What institutions can do and what students should learn. Washington, D.C.: American Council on Education.

Open Doors Data. (2016). Open Doors Annual Report [Institute of International Education]. Retrieved from http://iie.org/Research-and-Publications/OpenDoors/Data\#.WBozfOMrKUk 
Pellegrino, J., \& Hilton, M. (2012). Education for life and work: Developing transferable knowledge and skills in the 21st century (Committee on Defining Deeper Learning and 21st Century Skills). Washington, D.C.: National Research Council. Piaget, J. (1952). The origins of intelligence in children; New York, NY: International Universities Press.

Pike, G. (2008). Assessment matters: Learning about learning communities: Consider the variables. About Campus, 13(5), 30-32.

Pike, G., Kuh, G., \& McCormick, A. (2011). An investigation of the contingent relationships between learning community participation and student engagement. Research in Higher Education, 52(3), 300-322.

Prahalad, C. K. (1990). Globalization: The intellectual and managerial challenges. Human Resource Management, 29(1), 27-37.

Preston, M., \& Peck, A. (2016). Carts before horses? Remembering the primacy of the student's experience in student learning. New Directions for Student Leadership, (151), 79-91.

Provencher, A., \& Kassel, R. (2017). High-impact practices and sophomore retention: Examining the effects of selection bias. Journal of College Student Retention: Research, Theory \& Practice, 18(3), 1-21.

Purdie, J. R., \& Rosser, V. J. (2007). Examining the academic performance and retention offirst-year students in living-learning communities, freshmen interest groups and first year experience courses. University of Missouri-Columbia, Columbia, Mo.

Rath, T. (2007). StrengthsFinder (Version 2.0). New York, NY: Gallup Press. 
Reisweber, J. R. (2008). Beliefs, values, and the development of intercultural awareness (Unpublished dissertation). James Madison University, Harrisonburg, VA.

Richart, A. F. (2015). Effects of an international experience requirement, year in school, and preferred program duration on student interest in study abroad. Frontiers: The Interdisciplinary Journal of Study Abroad, (26), 17-29.

Rokeach, M. (1973). The nature of human values. New York, NY: Free Press.

Salisbury, M. (2015). Matching program and student characteristics with learning outcomes. In N. Sobania (Ed.), Putting the local in global education (pp. 36-51). Sterling, VA: Stylus Publishing, LLC.

Sandeen, C. (2012). High-Impact educational practices: What we can learn from the traditional undergraduate setting. Continuing Higher Education Review, 76.

Schneider, C. G. (2005). Making excellence inclusive: Liberal education \& America’s promise. Liberal Education, 91(2), 12-17.

Schwartz, S.H. (1992). Universals in the content and structure of values: Theoretical advances and empirical tests in 20 countries. In M. P. Zanna (Ed.), Advances in experimental social psychology (pp. 1-65). New York, NY: Academic Press.

Senge, P. (2006). The fifth discipline: The art and practice of the learning organization (2nd ed.). New York, NY: Double Day.

Senge, Peter, \& Kofman, F. (1995). Communities of commitment: The heart of learning organizations. In Learning organizations: Developing cultures for Tomorrow's Workplace (pp. 14-43). Portland, OR: Productivity Press. 
Shealy, C. N. (2004). A model and method for "making" a combined-integrated psychologist: Equilintegration (EI) theory and the Beliefs, Events, and Values Inventory (BEVI). Journal of Clinical Psychology, 60(10), 1065-1090.

Shealy, C. N. (2006). The Beliefs, Events, and Values Inventory (BEVI): Overview, implications, and guidelines. Harrisonburg, VA: Author.

Shealy, C. N. (Ed.). (2015). Making sense of beliefs and values: Theory, research, practice. New York, NY: Springer Publishing.

Sleeter, C., \& Grant, C. (1987). An analysis of multicultural education in the United States. Harvard Educational Review, 57(4), 421-445.

Soria, K., \& Troisi, J. (2014). Internationalization at home, alternatives to study abroad: Implications for students' development of global, international and intercultural competencies. Journal of Studies in International Education, 18(3), 261-280.

Stebleton, M., Soria, K., \& Cherney, B. (2013). The high impact of education abroad: College students' engagement in international experiences and the development of intercultural competencies. Frontiers Journal, 22, 1-24.

Sutton, R. C., \& Rubin, D. L. (2004). The GLOSSARI project: Initial findings from a system-wide research initiative on study abroad learning outcomes. Frontiers: The Interdisciplinary Journal of Study Abroad, 10, 65-82.

Taylor, E. W., \& Cranton, P. (Eds.). (2012). Reflecting back and looking forward. In The handbook of Transformative Learning: Theory, research, practice (pp. 555-573). San Francisco, CA: John Wiley \& Sons. 
Thakral, C., Vasquez, P. L., Bottoms, B. L., Matthews, A. K., Hudson, K. M., \& Whitley, S. K. (2016). Understanding difference through dialogue: A first-year experience for college students. Journal of Diversity in Higher Education, 9(2), 130-142.

Tillman, M. (2011). AIFS Student Guide to Study Abroad \& Career Development. AIFS. Retrieved from http://www.aifsabroad.com/advisors/pdf/Tillman_AIFS_Student_Guide_Career.p df

Tinto, V. (2006). Research and practice of student retention. Journal of College Student Retention: Research, Theory and Practice, 8(1), 1-9.

Twombly, S., Salisbury, M., Tumanut, S., \& Klute, P. (2012). Study Abroad in a New Global Century (Higher Education Report No. Volume 38 Report 4). Hoboken, New Jersey: Association for the Study of Higher Education.

University of California-Berkeley. (2014). SERU mission (Student Experience in the Research University). Berkeley, CA.

Vygotsky, L. (1997). Educational psychology. (R. Silverman, Trans.). Boca Raton, FL: St. Lucie Press.

Wandschneider, E., Pysarchik, D. T., Sternberger, L. G., \& Ma, W. (2015). The Forum BEVI project: Applications and implications for international, multicultural, and transformative learning. Frontiers: The Interdisciplinary Journal of Study Abroad, 25(Spring), 150-228.

Whitehead, D. M. (2015). Global learning: Key to making excellence inclusive. Liberal Education, (Summer), 6-13. 
Whitehead, D. M. (2016). Essential global learning. Washington, D.C.: Association of American Colleges and Universities.

Williams, D., Berger, J., \& McClendon, S. (2005). Toward a model of inclusive excellence and change in postsecondary institutions (White Paper; pp. 1-49). Washington, DC: Association of American Colleges and Universities.

Wilson-Mulnix, J. (2012). Thinking critically about critical thinking. Educational Philosophy and Theory, 44(5), 464-479.

Woolf, M. (2013, April 15). The Ideological Landscape of Education Abroad. (White Paper) Retrieved from http://capaworld.capa.org/2013/04/15/the-ideologicallandscape-of-education-abroad 


\section{VITA}

Jennifer L. Wiley was born in Springfield, Missouri, where she attended public schools through her high school career. Upon graduation from high school, Jennifer became the first person in her family to attend and complete a bachelor's degree. She attended the University of Missouri-Columbia and completed her Bachelor of Science degree in 1996.

After teaching for a few years, Jennifer pursued her Master of International Affairs and Administration degree at Missouri State University. Upon completion of that degree, Jennifer began serving as a visiting professor at Poltava State Agrarian University in Ukraine through the US Peace Corps. Through that experience and working in international education roles in higher education in the US, Jennifer joined with three female colleagues to establish the consulting firm she currently leads. 